Aus der Poliklinik für Präventive Zahnmedizin, Parodontologie und Kariologie (Prof. Dr. med. dent. A. Wiegand)

im Zentrum Zahn-, Mund- und Kieferheilkunde der Medizinischen Fakultät der Universität Göttingen

\title{
Die Wahrnehmung von Kindern mit Karies - eine Eye-Tracking-Studie
}

\author{
INAUGURAL-DISSERTATION \\ zur Erlangung des Doktorgrades \\ für Zahnheilkunde \\ der Medizinischen Fakultät der \\ Georg-August-Universität zu Göttingen
}

vorgelegt von

David Zimmermann

aus Höxter

Göttingen 2018 
Dekan:

Prof. Dr. rer. nat. H. K. Kroemer

Betreuungsausschuss

Betreuerin:

Prof. Dr. med. dent. A. Wiegand

Ko-Betreuerin:

PD Dr. rer. nat. K. Jordan

\section{Prüfungskommission}

Referentin:

Prof. Dr. med. dent. A. Wiegand

Ko-Referentin:

PD Dr. rer. nat. K. Jordan

Drittreferent:

Prof. Dr. med. M. Oppermann

Datum der mündlichen Prüfung: 08.10.2019 
Hiermit erkläre ich, die Dissertation mit dem Titel „Die Wahrnehmung von Kindern mit Karies - eine Eye-Tracking-Studie" eigenständig angefertigt und keine anderen als die von mir angegebenen Quellen und Hilfsmittel verwendet zu haben.

Göttingen, den 


\section{Inhaltsverzeichnis}

Abbildungsverzeichnis.............................................................................

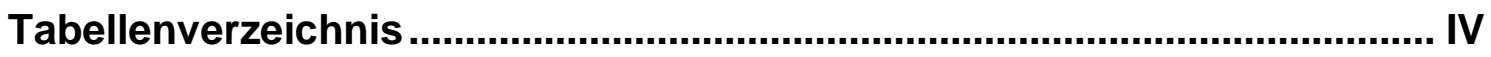

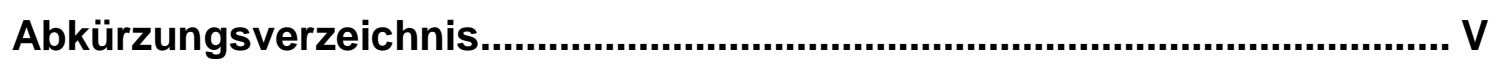

$1 \quad$ Einleitung

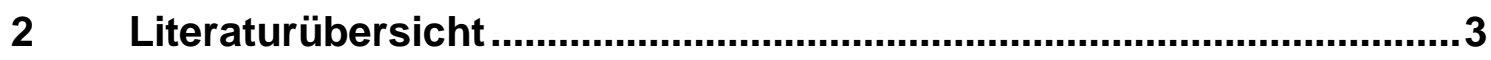

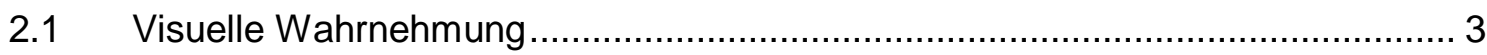

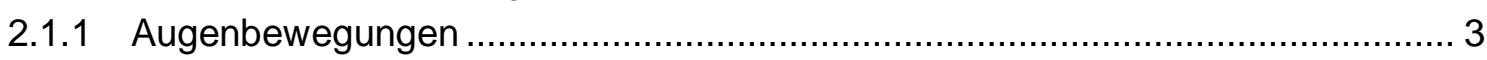

2.1.2 Aufzeichnung von Augenbewegungen ......................................................... 5

2.1.3 Interpretation von Augenbewegungen ........................................................ 7

2.1.4 Wahrnehmung von Gesichtern ................................................................ 8

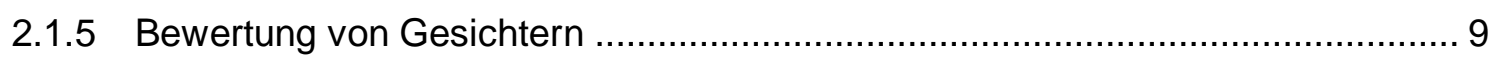

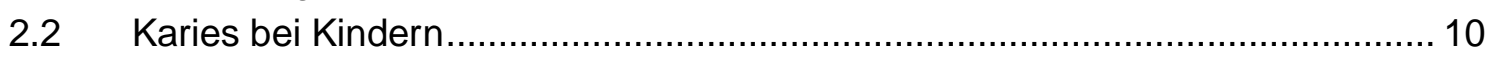

2.2.1 Early Childhood Caries ............................................................................ 12

2.2.2 Besonderheiten der Karies an Milchzähnen.................................................. 13

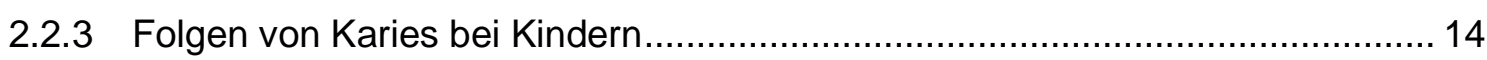

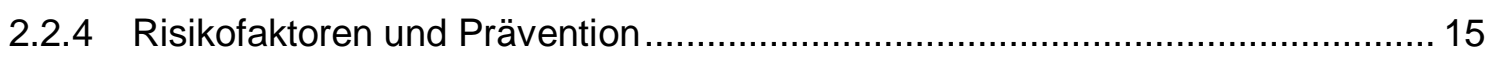

2.2.5 Epidemiologie von Karies bei Kindern in Deutschland .................................... 18

2.2.6 Soziale und sozioökonomische Folgen ........................................................ 18

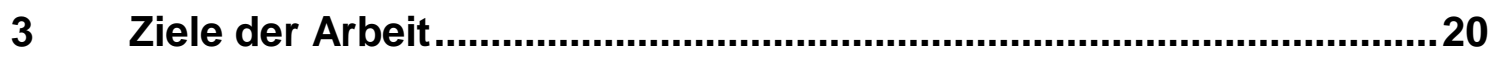

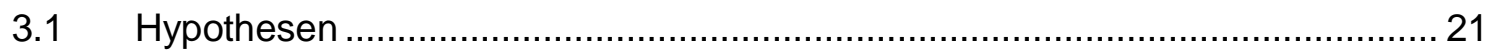

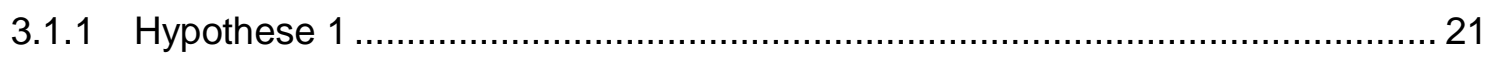

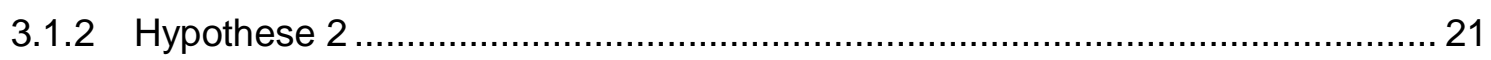

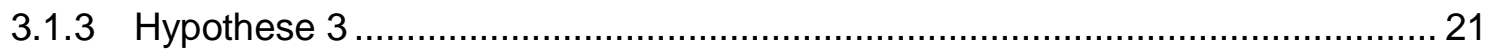

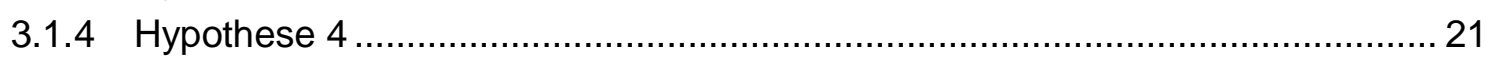

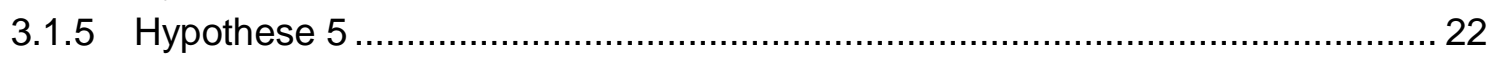

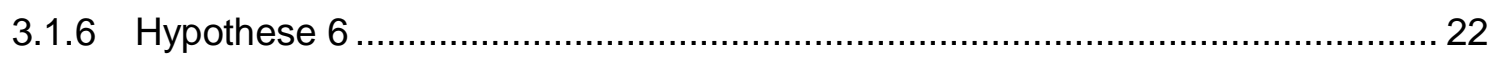

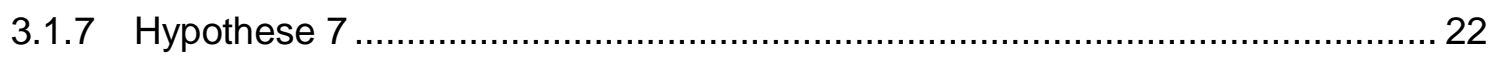

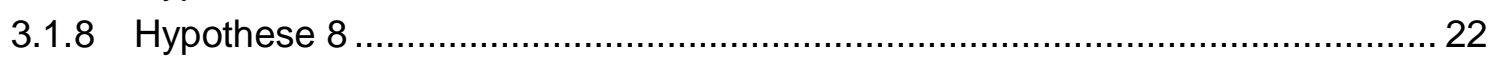

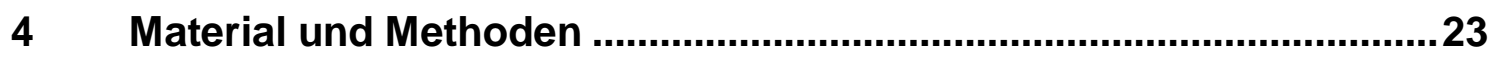

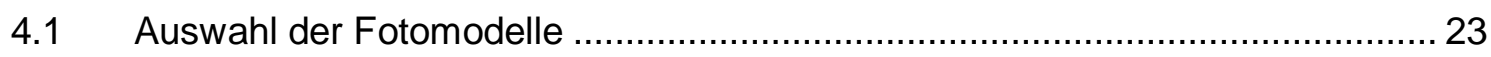

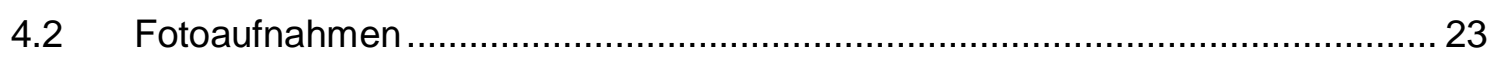

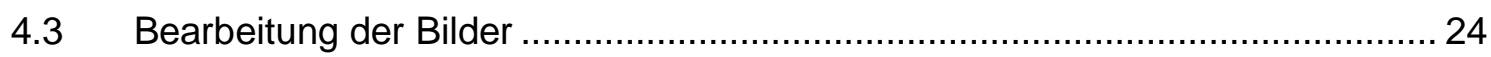

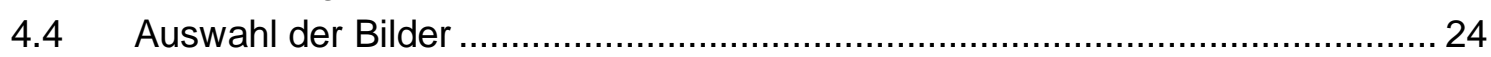

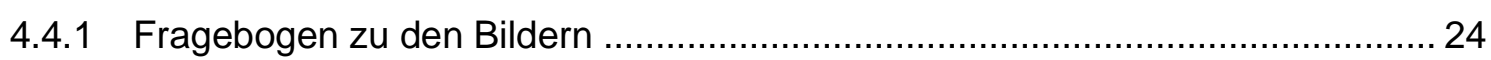

4.4.2 Vorauswahl der Fotos nach emotionalen Gesichtspunkten...............................2 25

4.4.3 Zahnmedizinische Einordnung …………………....................................... 25

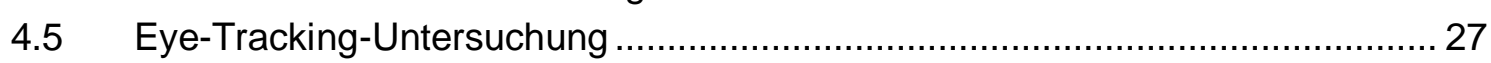

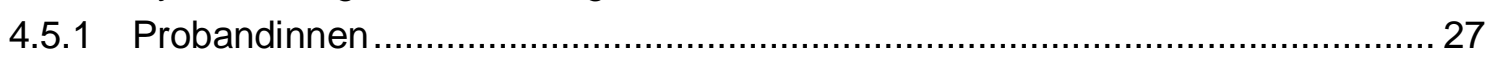

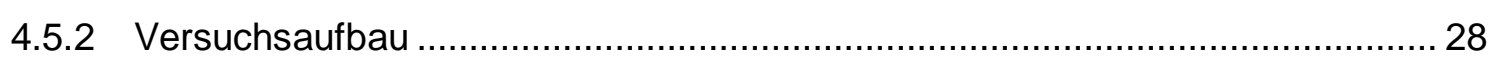




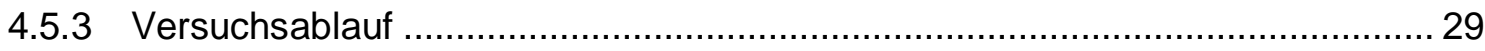

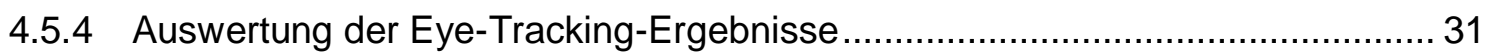

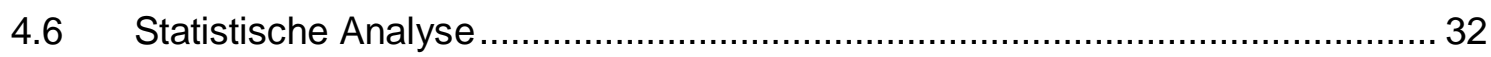

$5 \quad$ Ergebnisse..........................................................................................34

5.1 Wahrnehmung von Kindergesichtern durch zahnmedizinische Laien und

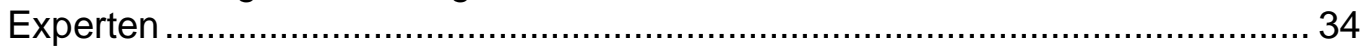

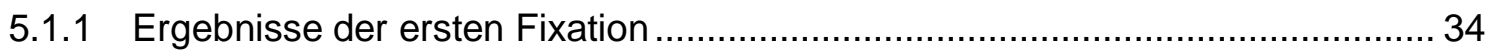

5.1.2 Ergebnisse der ersten bis zweiten Sekunde ................................................. 35

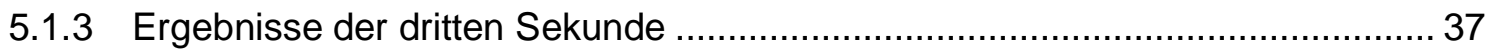

5.2 Wahrnehmung von Kindergesichtern in Abhängigkeit vom dentalen Befund ... 39

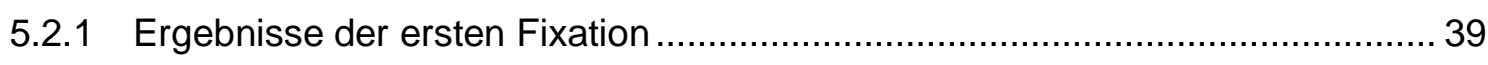

5.2.2 Ergebnisse der ersten bis zweiten Sekunde ............................................... 41

5.2.3 Ergebnisse der dritten Sekunde .............................................................. 44

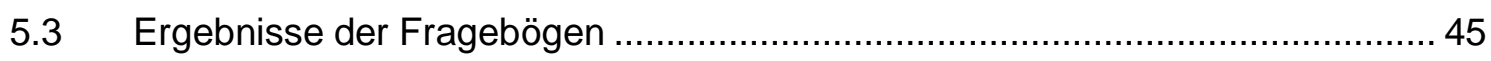

5.3.1 Vergleich der Probandinnen-Gruppen.......................................................... 45

5.3.2 Vergleich der Gebiss-Klassifikationen .................................................. 47

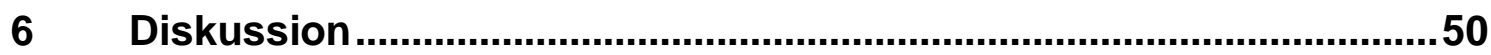

6.1 Diskussion der Ergebnisse ................................................................... 50

6.1.1 Wahrnehmung von Kindergesichtern durch zahnmedizinische Laien und

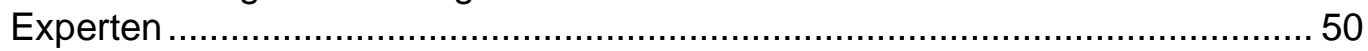

6.1.2 Wahrnehmung von Kindergesichtern in Abhängigkeit vom dentalen Befund ... 52

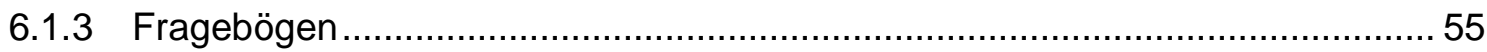

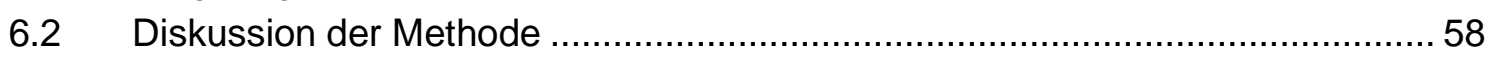

6.2.1 Fotos der Patienten und Auswahl der Fotos ................................................. 58

6.2.2 Durchführung der Eye-Tracking-Untersuchung …....................................... 59

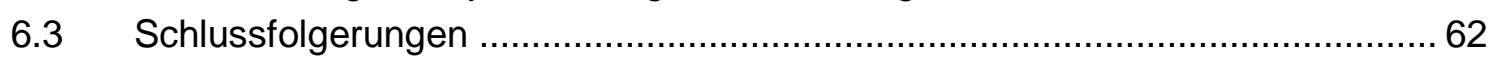

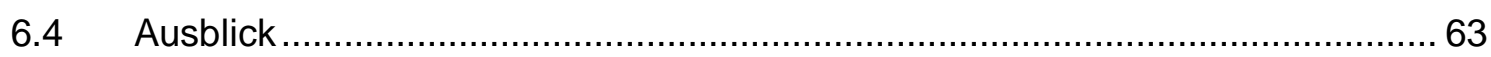

$7 \quad$ Zusammenfassung ........................................................................64

8 Literaturverzeichnis ...................................................................66

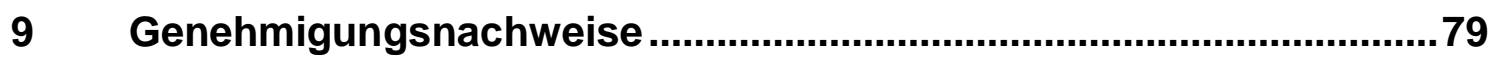

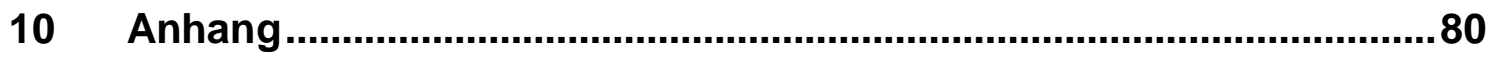




\section{Abbildungsverzeichnis}

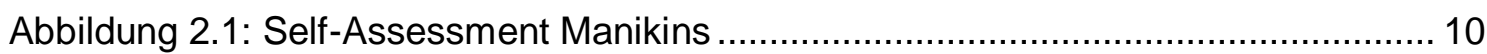

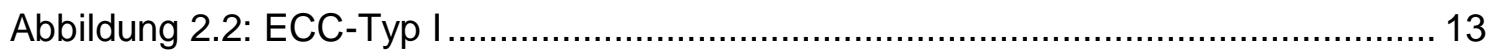

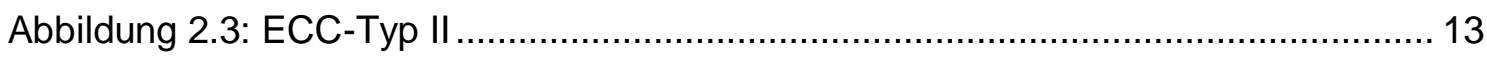

Abbildung 2.4: ECC-Typ III .................................................................................. 13

Abbildung 4.1: Flussdiagramm zur Auswahl und Verteilung der Patientenfotos .......... 26

Abbildung 4.2: Ablauf des Präsentationsbildschirms ................................................. 30

Abbildung 4.3: Beispiel eines Präsentationsbildes ...................................................... 31

Abbildung 5.1: Relative Häufigkeit (\%) der 1. Fixation zwischen den Probandinnen-

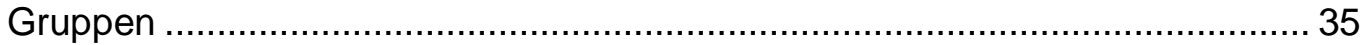

Abbildung 5.2: Mittelwerte der Gesamtfixationszeiten (ms) und $95 \%-K o n f i d e n z i n t e r v a l l$ für die 1. bis 2. Sekunde............................................ 36

Abbildung 5.3: Mittelwerte der Gesamtfixationszeiten (ms) und $95 \%-K o n f i d e n z i n t e r v a l l$ für die 3 . Sekunde ..................................................... 38

Abbildung 5.4: Relative Häufigkeit (\%) der 1. Fixation der Laien ................................. 40

Abbildung 5.5: Relative Häufigkeit (\%) der 1. Fixation der Experten ........................... 40

Abbildung 10.1: Fragebogen für die Probandinnen. .............................................. 80 


\section{Tabellenverzeichnis}

Tabelle 4.1: Verteilung der Bildpaare der Patienten hinsichtlich der verschiedenen

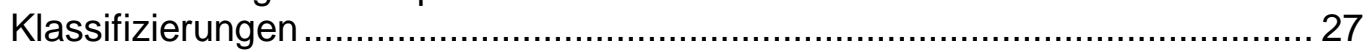

Tabelle 4.2: Einstellungen der Eye-Tracking-Analysesoftware SMI BeGaze ${ }^{\mathrm{TM}}$............. 32

Tabelle 5.1: Vergleich der Anzahl der Fixationen in der 1. bis 2. Sekunde zwischen

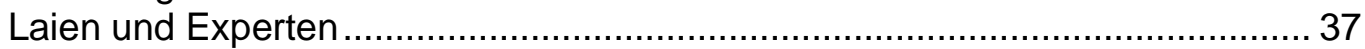

Tabelle 5.2: Vergleich der Anzahl der Fixationen in der 3. Sekunde zwischen Laien und Experten...

Tabelle 5.3: Vergleich der Gesamtfixationszeiten in der 1. bis 2. Sekunde zwischen den Gebiss-Klassifizierungen "gesund“, „Karies" und „Zahnsanierung“.

Tabelle 5.4: Vergleich der Anzahl der Fixationen in der 1. bis 2. Sekunde zwischen den Gebiss-Klassifizierungen „gesund“, „Karies“ und „Zahnsanierung“

Tabelle 5.5: Vergleich der Gesamtfixationszeiten in der 3. Sekunde zwischen den Gebiss-Klassifizierungen "gesund“, „Karies“ und „Zahnsanierung“.

Tabelle 5.6: Vergleich der Anzahl der Fixationen in der 3. Sekunde zwischen den Gebiss-Klassifizierungen „gesund“, „Karies“ und „Zahnsanierung“..

Tabelle 5.7: Valenz, Arousal und Attraktivität der Kinder mit gesunden Zähnen, mit kariösen Zähnen und mit Zahnsanierung beurteilt durch Laien und Experten, Vergleich der Probandinnen Gruppen ....

Tabelle 5.8: Valenz, Arousal und Attraktivität der Kinder mit gesunden Zähnen, mit kariösen Zähnen und mit Zahnsanierung beurteilt durch Laien und Experten, Vergleich der Gebiss-Klassifizierungen 


\section{Abkürzungsverzeichnis}

$\begin{array}{ll}\text { AOI } & \begin{array}{l}\text { area of interest } \\ \text { dmft-Index }\end{array} \\ & \begin{array}{l}\text { Summe der kariösen (decayed), aufgrund von Karies } \\ \text { extrahierten (missing) oder gefüllten (filled) Milchzähne } \\ (\text { teeth })\end{array} \\ \text { ECC } & \text { Early Childhood Caries } \\ \text { LKG-Spalten } & \text { Lippen-Kiefer-Gaumen-Spalten } \\ \text { ppm } & \text { parts per million } \\ \text { px } & \text { Pixel } \\ \text { RGB } & \text { Rot, Grün, Blau (Farbraum) } \\ \text { SAM } & \text { Self-Assessment Manikins }\end{array}$




\section{Einleitung}

Karies ist bei Kindern eine weit verbreitete Erkrankung, die mit starken Einschränkungen der Lebensqualität einhergehen kann (Agostini et al. 2001; Pieper 2010; Schiffner 2016). Karies im Frontzahnbereich kann ästhetische und sprachliche Einschränkungen hervorrufen, die zu Ausgrenzungen durch Mitmenschen führen können (Shaw 1981; Filstrup et al. 2003; Karunakaran et al. 2011). Studien bei Erwachsenen konnten zeigen, dass Fotos von Gesichtern mit kariösen Zähnen als unattraktiver bewertet werden als Gesichter mit gesunden Zähnen (Kershaw et al. 2008). Wenn die Bewertungen von Gebiss-Fotos durch zahnmedizinische Laien und Experten verglichen werden, zeigt sich, dass Experten kritischer sind und schon kleine Veränderungen der Zähne als störend empfinden (Kokich et al. 1999; Kokich et al. 2006).

Eye-Tracking ist ein bewährtes Verfahren, um Augenbewegungen aufzuzeichnen, dabei können die Dauer und die Lokalisation von Blicken analysiert werden (Buswell 1935; Yarbus 1967). Aus dem untersuchten Blickverhalten lassen sich Rückschlüsse auf die visuelle Aufmerksamkeit ziehen (Findlay und Gilchrist 1998; Hoffman 1998). In der Medizin wurde Eye-Tracking bisher unter anderem dazu eingesetzt, die Wahrnehmung von Schädel- und Gesichtsanomalien zu erforschen. Dabei konnte dargelegt werden, dass anatomische Veränderungen $z u$ einem differenten Betrachtungsverhalten der Probanden führen (Meyer-Marcotty et al. 2010b; Litschel et al. 2015; van Schijndel et al. 2015).

Bisherige Studien, die die Wahrnehmung von Zähnen oder Gesichtern mittels Eye-Tracking analysierten, konzentrierten sich auf kieferorthopädische Fehlstellungen. Hierbei wurde festgestellt, dass die Mundregion von Patienten mit starkem kieferorthopädischen Behandlungsbedarf eine große visuelle Aufmerksamkeit auf sich zieht (Meyer-Marcotty et al. 2010a; Richards et al. 2015; Baker et al. 2018).

Einige Autoren konnten nachweisen, dass pathologische zahnärztliche Befunde, insbesondere Karies, zu sozialer Ausgrenzung und Ablehnung führen. Patienten mit sichtbarer Karies wurde eine geringe Sozialkompetenz und Intelligenz zugesprochen (Somani et al. 2010). Ebenso wurden sie als unattraktiver und unbeliebter bewertet als gesunde Patienten (Karunakaran et 
al. 2011). Kinder, die selbst an Karies erkrankt waren, gaben an, aufgrund ihrer Zähne gehänselt worden zu sein (Filstrup et al. 2003).

Über die Wahrnehmung von kindlicher Karies liegen noch keine Untersuchungen, im Besonderen noch keine Eye-Tracking-Untersuchungen, vor. Es ist unklar, ob unterschiedliche Zahnbefunde (gesundes Gebiss, Karies und Zahnsanierung) das Betrachtungs- und Bewertungsverhalten beeinflussen. Daher zielte die vorliegende Studie darauf ab, mittels Eye-Tracking zu untersuchen, wie Kinder mit sichtbarer Karies im Vergleich zu Kindern nach Zahnsanierung (Füllungen, Extraktionen und/oder Stahlkronen) oder mit gesunden Zähnen durch zahnmedizinische Laien und Experten wahrgenommen werden, und wie diese Kinder anschließend hinsichtlich Valenz, Arousal und Attraktivität bewerten werden. Darüber hinausgehend wurden in der Literatur Unterschiede in der Betrachtung und Bewertung von Gesichtern durch Laien und Experten beschrieben (Kokich et al. 2006; Dindaroğlu et al. 2017). Deshalb war ein weiteres Ziel der Studie festzustellen, ob es Unterschiede in der Wahrnehmung der Kinder zwischen zahnmedizinische Laien und Experten gibt. 


\section{Literaturübersicht}

\subsection{Visuelle Wahrnehmung}

Die visuelle Wahrnehmung, das Sehen, beschreibt das Auftreffen von elektromagnetischen Wellen im sichtbaren Bereich auf die Retina, der Weiterleitung der daraus resultierenden neuronalen Signale und deren Verarbeitung im Gehirn (Bellebaum et al. 2012). Der Mensch sieht nur scharf in der Fovea centralis und in einem Bereich von $1^{\circ}$ Sehwinkel um sie herum (Sibert und Jacob 2000). Die hervorragende Schärfe dort resultiert aus der hohe Dichte an kleinen rezeptiven Feldern in der Region der Fovea centralis (Hubel 1995). Ab einer Abweichung von nur $3^{\circ}$ Sehwinkel zur Fovea centralis verringert sich die Sehschärfe bereits um die Hälfte (Joos et al. 2003). Die Erregungen der Nervenzellen in der Retina werden über die Sehbahnen in den primär visuellen Kortex weitergeleitet und von dort in andere visuelle Areale projiziert (Bellebaum et al. 2012).

\subsubsection{Augenbewegungen}

Joos et al. (2003) haben drei Gründe definiert, warum es zu einer Bewegung der Augen kommen kann: (1) Es wird ein neues Objekt fixiert und der Blick wandert mittels Sakkaden dorthin. (2) Das betrachtete Objekt bewegt sich und die Augen folgen der Bewegung. Bei schnellen Bewegungen des Objektes sind Sakkaden nötig, um dem Objekt zu folgen. (3) Der Körper des Betrachters bewegt sich während des Sehvorganges eines Objekts und das menschliche Auge kompensiert diese Bewegung. Auf diese Weise findet ein ständiger Wechsel von Fixationen und Sakkaden statt.

\subsubsection{Sakkaden}

Der Begriff Sakkade leitet sich aus dem Französischen ab und bedeutet so viel wie ruckweise oder abgehackt (Brockhaus 2000). Er definiert die Augenbewegungen zwischen zwei Fixationen, die dazu dienen, die Fovea centralis auf ein neues Ziel zu richten (Duchowski 2007). Während einer Sakkade wird das Gesehene nur zu einem geringen Teil wahrgenommen (Volkmann 1962), sodass eine Reaktion auf das Gesehene nicht möglich ist 
(Carpenter 1977). Daher können Sakkaden nicht mehr unterbrochen oder in ihrer Richtung geändert werden, wenn sie einmal begonnen wurden (Duchowski 2007). Sie werden von beiden Augen nahezu in gleicher Richtung und Geschwindigkeit ausgeführt (Krauskopf et al. 1960). Die Dauer von Sakkaden liegt in der Regel zwischen 10 bis 100 ms (Duchowski 2007; Nyström und Holmqvist 2010) und hängt linear von der Weite des Sehsprunges ab (Carpenter 1977). Sie erreichen dabei Winkelgeschwindigkeiten von bis zu 1000 \% (Boff und Lincoln 1988). Die Winkelgeschwindigkeit bei der Ausführung einer Sakkade nimmt zu Beginn stark zu und erreicht bereits vor der Hälfte der Bewegung ihr Maximum, um dann wieder langsam abzunehmen (Boumans et al. 1980).

\subsubsection{Fixationen}

Fixationen sind Augenbewegungen, bei denen sich die Augen in relativer Ruhe zum angeschauten Objekt befinden. Während einer Fixation stehen die Augen nicht vollkommen still, sondern werden minimal bewegt (Joos et al. 2003). Diese minimalen Bewegungen werden in drei verschiedene Arten unterteilt: Tremor, Drift und Mikrosakkade. Als Tremor wird der Zustand des leichten Zitterns der Augenmuskulatur von unter einer Winkelminute mit einer Frequenz von etwa $50 \mathrm{~Hz}$ bezeichnet (Wolf und Wolf 1990). Bei den Driften kommt es zur Verschiebung des Fixationspunktes auf der Retina um einige Sehzellen. Mikrosakkaden sind Sprünge zurück zum Fixationspunkt, um vorausgegangene Driftbewegungen auszugleichen (Rötting 1999a; Joos et al. 2003). Sie haben eine Amplitude von ein bis zwei Winkelminuten (Duchowski 2007). Diese drei Arten von Bewegungen dienen dazu, eine Adaptation der Nervenzellen der Retina zu verhindern und somit zu einem guten Seheindruck zu führen. Sie werden nicht bewusst wahrgenommen oder ausgeführt (Carpenter 1977; Duchowski 2007). Die Dauer von Fixationen ist unterschiedlich lang und variiert je nach Betrachtungsaufgabe, beispielsweise zwischen dem Lesen von Texten und der Betrachtung von Bildern (Nyström und Holmqvist 2010). Die minimale Dauer einer Fixation wird in der Literatur von einigen Autoren mit mindestens 100 ms angegeben (Young und Sheena 1975; Karsh und Breitenbach 1983), die Länge einer durchschnittliche Fixation mit 200 bis 600 ms (Sibert und Jacob 2000). Es werden jedoch auch sehr kurze Fixationen, sogenannte 
Expressfixationen, mit einer Dauer von unter $50 \mathrm{~ms}$ bis maximal $100 \mathrm{~ms}$ beschrieben (Velichkovsky et al. 1997, Nyström und Holmqvist 2010).

\subsubsection{Beeinflussung der visuellen Wahrnehmung}

Verschiedene Faktoren können einen Einfluss auf die visuelle Wahrnehmung haben. Dazu zählen unter anderem die Händigkeit, das Geschlecht, das Alter, die Einnahme von Medikamenten oder Erkrankungen, die die neurophysiologische Reizverarbeitung beeinflussen. Rechtshänder weisen beispielsweise eine pseudoneglect auf. Dies bedeutet, dass sie eine Tendenz haben, mehr nach links als nach rechts zu schauen. Dieses Verhalten ist bei Linkshändern weniger stark ausgeprägt (Bowers und Heilman 1980; Nicholls et al. 2017). Die Einnahme von Alkohol und Drogen, wie zum Beispiel Cannabis, verlängert die Fixationsdauer und steigert die Anzahl der Fixationen (Moser et al. 1998; Huestegge et al. 2010; Silva et al. 2017). Patienten mit neurologischen Erkrankungen, wie zum Beispiel Autismus, weisen bei der Betrachtung von Gesichtern ein verändertes Wahrnehmungsmuster auf. Im Vergleich zu gesunden Probanden zeigen sie ein geringeres Interesse für die Gesichtsregionen Augen, Nase und Mund, stattdessen werden andere Teile des Gesichtes häufiger betrachtet (Pelphrey et al. 2002).

\subsubsection{Aufzeichnung von Augenbewegungen}

Die Bewegungsabläufe und Geschwindigkeiten der Augenbewegungen begann man bereits sehr früh zu erforschen (Lamansky 1869). Im Laufe der Geschichte wurden dazu verschiedene Methoden entwickelt. Früher wurde diese Techniken häufig unter dem Begriff der Okulographie zusammengefasst (Schmöger 1972). Heute wird meist der englische Begriff Eye-Tracking verwendet (Duchowski 2007).

\subsubsection{Methoden zur Aufzeichnung von Augenbewegung}

Im 16. Jahrhundert beschränkte sich die Beobachtung der Augenbewegung noch auf die direkte Betrachtung eines Gegenübers (Laurentius 1599). Hierbei konnten Bewegungen ab $1^{\circ}$ Sehwinkel erkannt werden (Rötting 1999b).

Die ersten technischen Aufnahmen fanden Anfang des 20. Jahrhunderts mit einer Kontaktlinse statt, in die ein Spiegel eingearbeitet war. Er wurde mit Licht 
beschienen und warf dieses zurück. Die Reflektionen wurden mittels einer Kamera aufgezeichnet (Dodge 1907).

Das coil system nutzt eine Kontaktlinse, in welche Metallspulen eingearbeitet sind. Um die Position des Auges feststellen zu können, wird rund um den Kopf des Probanden in allen drei Raumebenen ein elektromagnetisches Feld erzeugt, welches sich mit den Bewegungen der Augen verändert (Joos et al. 2003). Diese Methode erreicht sehr hohe Genauigkeiten mit 5 bis 10 Winkelsekunden, allerdings ist die Größe des Blickfeldes technisch bedingt sehr eingeschränkt (Young und Sheena 1975).

Eine weitere Entwicklung in der Aufzeichnung von Augenbewegungen war das Elektro-Okulogramm bzw. die Elektro-Okulographie. Dabei werden Spannungsdifferenzen im $\mu \mathrm{V}$-Bereich zwischen Cornea und Retina gemessen, die sich mit der Bewegung des Auges ändern (Mowrer et al. 1935). Rund um das Auge des Probanden werden vier Elektroden, je ein Paar für horizontale und vertikale Auslenkungen, angebracht, die die Spannungsdifferenzen messen und daraus die Position des Auges bestimmen (Oster und Stern 1980). Die Genauigkeit des Systems beträgt in etwa $\pm 2^{\circ}$ Sehwinkel bei einem Blickfeld von $30^{\circ}$ (Shackel 1967; Mickasch und Haack 1986). Die Elektro-Okulographie ist sehr fehleranfällig (Schmöger 1972).

Die Cornea-Reflex-Methode oder dual-purkinje image nutzt das erste und vierte Purkinje-Bild. Insgesamt gibt es im menschlichen Auge vier Purkinje-Bilder, die durch die Brechung des Lichtes an Grenzflächen zustande kommen. Das erste Purkinje-Bild, auch Cornealreflex genannt, entsteht an der Grenzfläche zwischen Luft und Cornea (Hornhaut), das zweite zwischen Cornea und Humor aquosus (Kammerwasser), das dritte zwischen Humor aquosus und Lens (Linse), das vierte zwischen Lens und Corpus vitreum (Glaskörper) (Crane und Steele 1978). Bei der Bewegung des Auges kommt es zu einer Verschiebung der Purkinje-Bilder. Sie verschieben sich unterschiedlich stark, wodurch sich die Position des Auges errechnen lässt (Duchowski 2007). Diese Methode wurde bereits 1910 beschrieben, jedoch aufgrund ihrer Komplexität für nicht praktikabel erachtet, da für die Berechnung die individuelle Krümmung der Cornea berücksichtigt werden muss (von Helmholtz 1910b). 
Im Rahmen moderner Eye-Tracking-Untersuchungen wird für die Blickachsenmessung (point of regard measurement) das Auge mit Infrarotlicht beschienen. Aus dem Cornealreflex, der Pupillenmitte und einem definierten Abstand zum Präsentationsbildschirm kann die Blickachse berechnet werden, die sich bei Bewegungen des Auges verändert (Mickasch und Haack 1986; Joos et al. 2003). Die Methode wird als dark-pupil-Methode bezeichnet, da das Infrarotlicht von der Pupille absorbiert wird und diese schwarz erscheint. Abzugrenzen davon ist die bright-pupil-Methode, bei der die Lichtquelle senkrecht auf das Auge gerichtet wird, sodass das einfallende Licht den Augenhintergrund erleuchtet und die Pupille hell erscheint (Joos et al. 2003).

\subsubsection{Interpretation von Augenbewegungen}

Für die Eye-Tracking-Forschung sind zwei Thesen von Just und Carpenter (1980) von essenzieller Bedeutung. Sie haben die Unmittelbarkeits-Annahme (immediacy assumption) und die Auge-Geist-Annahme (eye-mind assumption) aufgestellt. Die Unmittelbarkeits-Annahme besagt, dass die Information des angeschauten Objekts direkt verarbeitet wird. Der Auge-Geist-Annahme nach dauert eine Fixation so lange, wie über das fixierte Objekt nachgedacht wird. Diese Theorien des direkten Zusammenhangs zwischen fovealem Sehen und kognitiver Verarbeitung konnten in weiteren Studien bestätigt werden (Hoffman 1998; Findlay und Gilchrist 2003).

Ebenso ist es möglich, die Aufmerksamkeit auf Bereiche zu legen, die außerhalb der Fovea centralis liegen, was als parafoveales Sehen bezeichnet wird (Posner 1980; Carrasco 2011). Frühe Studien durch von Helmholtz (1910a) zeigen, dass Probanden, die einen bestimmten Punkt fixieren, Licht außerhalb des Fixierten bewusst wahrnehmen können. Zugleich sind Probanden in der Lage, Objekte zu erkennen und zu benennen, die parafoveal angeschaut werden, gleichwohl ist es deutlich schwieriger als foveales Sehen (Henderson et al. 1997). Die foveale Betrachtung wird als offene Aufmerksamkeit (overt attention) bezeichnet. Im Gegensatz dazu wird parafoveales Sehen verdeckte Aufmerksamkeit (covert attention) genannt (Itti und Koch 2001; Carrasco 2011). Das parafoveale Sehen scheint an der Planung von neuen Sakkaden und Fixationen beteiligt zu sein (Henderson et al. 1989). Insbesondere bei kognitiv anspruchsvollen Aufgaben ist es für 
Probanden schwierig, diese mithilfe von verdeckter visueller Aufmerksamkeit zu lösen (Duncan und Humphreys 1989; He und Kowler 1992). Probanden, die nicht entsprechend instruiert wurden, zeigten keine Anzeichen von verdeckter visueller Aufmerksamkeit (Findlay und Gilchrist 1998).

Andere Studien haben sich mit der Thematik befasst, wie die Blickbewegungsparameter „Fixationsanzahl“ und „Fixationsdauer" zu bewerten sind. Untersuchungen, in denen sich Probanden später an gezeigte Inhalte erinnern sollten, konnten darlegen, dass unter dieser Aufgabenstellung sowohl die Anzahl als auch die Länge der Fixationen stieg (Loftus 1972; Tversky 1974). Auch werden Objekte länger und häufig als erstes fixiert, die im Kontext eines Bildes nicht erwartet werden, beispielsweise wenn ein Münztelefon in einer Küche dargestellt wird (Loftus und Mackworth 1978; Antes und Penland 1981; Friedman und Liebert 1981). Daraus kann geschlussfolgert werden, dass die kognitive Verarbeitung des Fixierten in solchen Fällen länger dauert. In Bezug auf das Betrachten von Gesichtern gibt es die Theorie, dass beim Vergleichen von mehreren Gesichtern die Fixationen länger sind als bei der Betrachtung von nur einem Gesicht (Duchowski 2007). Insbesondere der ersten Fixation wird eine besondere Rolle zugesprochen, da sie als Marker der initialen Aufmerksamkeit herangezogen werden kann (Nummenmaa et al. 2006).

Für die vorliegenden Studie wird das Betrachtungsverhalten von Probandinnen auf Kindergesichtern untersucht, was sich als Variable nicht direkt erfassen lässt. Jedoch sind die Augenbewegungen in Form von Anzahl, Dauer und Lokalisation der Fixationen messbar. Mithilfe der angeführten Augenbewegungsparameter kann das Betrachtungsverhalten operationalisiert werden.

\subsubsection{Wahrnehmung von Gesichtern}

Studien hinsichtlich des Anschauverhaltens von Gesichtern belegten, dass den Regionen Augen, Nase und Mund eine besondere Aufmerksamkeit zuteilwird (Yarbus 1967; Henderson et al. 2005). Die Blickpfade bei der Betrachtung von Gesichtern verlaufen in der Regel von den Augen über die Nase zum Mund, wobei die Augen die wichtigste Rolle einnehmen und maßgeblich zuerst angesehen werden (Walker-Smith et al. 1977; Hickman et al. 2010). 


\subsubsection{Einflussfaktoren auf die Gesichtswahrnehmung}

Ebenso wie die allgemeine visuelle Wahrnehmung wird auch die Wahrnehmung von Gesichtern durch verschiedene Faktoren beeinflusst. Unter anderem ist das Geschlecht des Betrachters wichtig für das Anschauverhalten. Frauen betrachten Gesichter im Vergleich zu Männern anders. Sie legen einen stärkeren Fokus auf die Augenregion, wohingegen Männer häufiger auf die Gesichtsregionen Nase und Mund schauen (Vassallo et al. 2009; Hall et al. 2010). Die Symmetrie von Gesichtern ist ebenfalls stark beeinflussend. So werden bei Patienten mit Lippenspalten die asymmetrischen Gesichtsregionen sowohl überproportional häufiger als auch länger angeschaut (Meyer-Marcotty et al. 2010b; Meyer-Marcotty et al. 2011; van Schijndel et al. 2015). Gleiches zeigt sich bei Kindern mit Schädeldeformationen, deren Asymmetrien länger betrachtet werden (Linz et al. 2016). Auch abstehende Ohren ziehen vermehrt Aufmerksamkeit auf sich und werden folglich länger angesehen (Haworth et al. 2015; Litschel et al. 2015). In einer anderen Studie wurde die Wahrnehmung von Patienten mit Lippenspalten durch Kieferorthopäden und Laien untersucht. Es zeigte sich, dass Experten die Patienten intensiver betrachteten als Laien (Dindaroğlu et al. 2017). Daneben spielt zusätzlich der Gesichtsausdruck der betrachteten Person eine große Rolle, welche Gesichtsregion am häufigsten angeschaut wird. Bei neutralen Gesichtsausdrücken werden die Augen und der Mund gleich häufig betrachtet. Bei traurigen Gesichtsausdrücken verschiebt sich die Anzahl der Fixationen auf die Augen (Eisenbarth und Alpers 2011; Schurgin et al. 2014).

\subsubsection{Bewertung von Gesichtern}

Wie bereits oben dargelegt, werden Gesichter mit emotionalem Gesichtsausdruck anders betrachtet als Gesichter mit neutralem Ausdruck. Um herauszufinden, welche Stärke die hervorgerufenen Emotionen haben, können sie hinsichtlich der zwei Dimensionen „Valenz" und „Arousal" bewertet werden (Adolph und Alpers 2010). Diese leiten sich aus dem circumplex model von Russell (1980) ab. Der Begriff Valenz beschreibt in der Psychologie die Wertigkeit oder Stärke eines Eindruckes und wird in den Dimensionen positiv, neutral oder negativ angegeben (Dorsch 2017b). Alternativ können auch die Worte „Wohlgefallen“ oder „Vergnügen“ genutzt werden (Franke 2014). Arousal 
beschreibt die Erregung, die durch die Betrachtung ausgelöst und aufsteigend von wenig bis stark angegeben wird (Adolph und Alpers 2010). Um die beiden Emotionen abzubilden, eignen sich die von Lang (1980) entwickelten Self-Assessment Manikins (Selbsteinschätzungs-Männchen, Abbildung 2.1) (Bradley und Lang 1994).

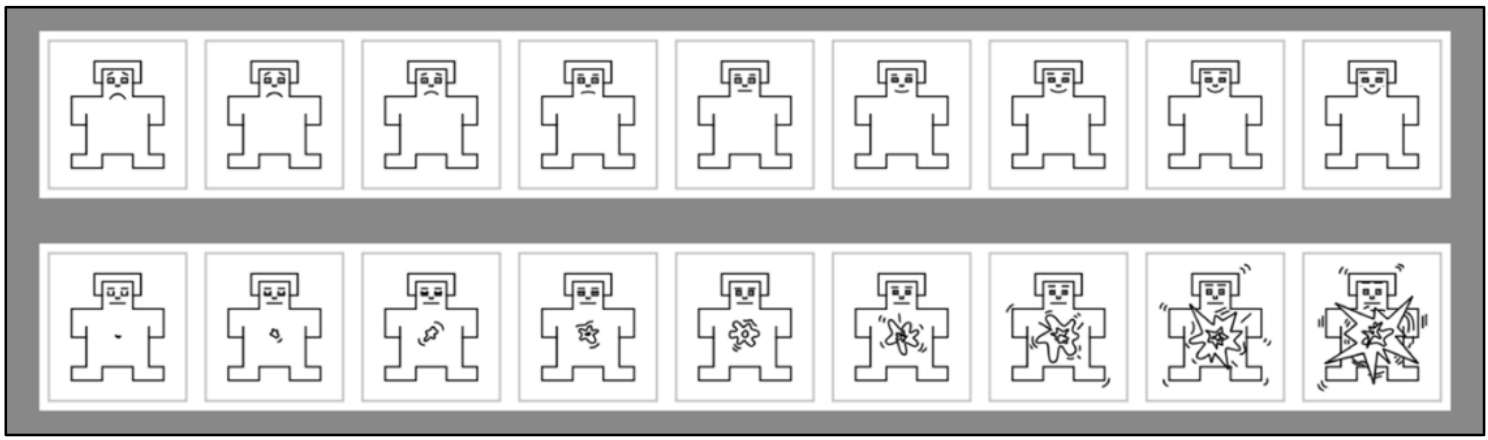

Abbildung 2.1: Self-Assessment Manikins (Lang 1980) für Valenz (oben) und Arousal (unten) modifiziert nach Irtel (2007), die Verwendung erfolgt mit freundlicher Genehmigung des Autors, Nachweis auf Seite 79.

Bei physischer Attraktivität handelt es sich um ein subjektives Kriterium, das zur Messung von Schönheit eingesetzt wird (Hassebrauck 1983). Bilder von Patienten mit einem fehlenden Frontzahn wurden im Gegensatz zu vollbezahnten Patienten als unattraktiver bewertet. Es machte keinen Unterschied, ob die Bewertung von zahnmedizinischen Laien oder Experten vorgenommen wurde (Oosterhaven et al. 1989). Andere Studien zur Attraktivitätsbewertung von Patienten mit unterschiedlich stark ausgeprägten dentalen Symmetrieabweichungen konnten hingegen Differenzen zwischen zahnmedizinischen Laien und Experten aufzeigen: Experten bewerteten bereits geringe Abweichungen als unattraktiv, wohingegen Laien erst durch stärkere Abweichungen in ihrer Bewertung beeinflusst wurden (Kokich et al. 1999; Kokich et al. 2006).

\subsection{Karies bei Kindern}

Die Erkrankung Karies beschreibt die Zerstörung der Zahnhartsubstanzen Schmelz, Dentin und Zement. Karies ist eine multifaktorielle Erkrankung, die sich auf verschiedene Ursachen zurückführen lässt (Fejerskov 2004). Das aktuell favorisierte Modell zur Beschreibung der Kariesentstehung basiert auf der ökologischen Plaquehypothese. Sie besagt, dass es pathogene und 
protektive Faktoren in der Mundhöhle gibt, die an der Entstehung von Karies beteiligt sind. In der gesunden Mundhöhle herrscht ein Gleichgewicht dieser Faktoren. Wenn es zu einer Verschiebung des Gleichgewichtes kommt und die pathogenen Faktoren überwiegen, entsteht Karies (Takahashi und Nyvad 2008; Paris und Meyer-Lückel 2012).

Zu den pathogenen Faktoren zählen hauptsächlich die im Mund vorhandenen Mikroorgansimen und die von innen verstoffwechselbaren Kohlenhydrate (Zero 2004). Bisher sind über 700 Mikroorganismen aus der Mundhöhle erforscht worden, welche sich auf (Zahn-)Oberflächen in extrazellulärer Matrix organisieren und sogenannte dentale Biofilme, Plaque genannt, bilden (Hojo et al. 2009; Dewhirst et al. 2010; Verma et al. 2018). Wird über die Nahrung ein hoher Anteil an niedermolekularen Kohlenhydraten aufgenommen, kommt es zu einer Verschiebung der physiologischen Zusammensetzung der Bakterien. Die Bakterien, die Zucker zu Säuren metabolisieren und darüber hinaus im sauren Milieu überleben können, haben einen Selektionsvorteil (Marsh 1994; Takahashi und Nyvad 2016). Durch die produzierten Säuren kommt es zu einer Senkung des pH-Wertes in der Plaque (Hirzel 1969; Cole und Eastoe 2014). Fällt dieser unter den kritischen Wert, er liegt für Schmelz bei pH 5,5, werden aus dem Hydroxylapatit einzelne lonen herausgelöst (Demineralisation) (Schmidt-Nielsen 1946; Dawes 2003). Ionen können sich in das Hydroxylapatit einlagern (Remineralisation), wenn bei steigendem $\mathrm{pH}$-Wert die lonenkonzentration des Speichels übersättigt ist. Übersteigt die Demineralisation die Remineralisation, kommt es zu einem lonenverlust und am Zahn entstehen irreversible Schäden (Fejerskov 2015). Dieser Prozess und das damit verbundene Fortschreiten von Karies bis zur völligen Zerstörung der Zahnhartsubstanzen vollzieht sich innerhalb von Monaten und Jahren (Shwartz et al. 1984).

Als protektive Faktoren werden die Immunabwehr des Patienten und seine Mundhygienemaßnahmen angesehen, welche zu einer Dezimierung der Bakterien und der Entfernung des Biofilms führen (Paris und Meyer-Lückel 2012). Die Remineralisation wird insbesondere durch den Speichel und die Zufuhr von Fluoriden gefördert (Fox 2004; Fejerskov et al. 2015). 


\subsubsection{Early Childhood Caries}

Early Childhood Caries (ECC) liegt bei Kindern bis zu 71 Monaten vor, wenn ein oder mehrere Zähne kariös, aufgrund von Karies verloren gegangen sind oder gefüllt werden mussten (American Academy of Pediatric Dentistry 2003). Der amerikanische Arzt Abraham Jacobi beschrieb 1862 als Erster das klinische Bild von frühkindlicher Karies und nannte als mögliche Ursache für Karies bereits Zucker (Jacobi 1862). Im Laufe der Zeit wurden für die frühkindliche Karies verschiedene Begriffe verwendet. So finden sich in der deutschsprachigen Literatur die Bezeichnungen Zuckertee-Karies (Wetzel 1982) oder Zucker-Saugerflaschen-Syndrom (Wetzel 1988). Im englischsprachigen Raum sind unter anderem die Begriffe nursing bottle mouth (Fass 1962), nursing-bottle syndrome (Babeely et al. 1989) oder nursing caries (Marchant et al. 2001) zu finden. Diese Ausdrücke legen jedoch nahe, dass die Ernährung alleiniger Auslöser sei, weshalb Fachgesellschaften wie die „Centers for Disease Control and Prevention“ seit 1994 die Verwendung des Begriffs early childhood caries empfehlen (Kaste 1995; Davies 1998). Im Deutschen wird dies mit frühkindlicher Karies übersetzt und heute üblicherweise verwendet (Bekes 2017).

\subsubsection{Einteilung der Early Childhood Caries}

Nach Wyne (1999) wird frühkindliche Karies in drei Gruppen klassifiziert. Bei Typ I existieren isolierte kariöse Läsionen an Molaren und/oder Inzisiven (Abbildung 2.2). Dieser Typ wird häufig durch feste und halbfeste kariogene Nahrung sowie durch mangelnde Mundhygiene hervorgerufen. Hauptsächlich sind Kinder zwischen zwei und fünf Jahren betroffen. Patienten mit Typ II weisen kariöse Läsionen an den vestibulären und palatinalen Flächen der Oberkieferinzisiven auf, die Unterkieferinzisiven sind nicht betroffen, die Molaren im Ober- und Unterkiefer können betroffen sein (Abbildung 2.3). Die Ursache liegt häufig in einer falschen Ernährung, zumeist in Kombination mit fehlender Mundhygiene. Die Typ II Karies tritt oftmals kurz nach dem Durchbruch der ersten Milchzähne auf und kann sich zu Typ III entwickeln, wenn keine adäquate Therapie und Ernährungsumstellung stattfinden. Bei Typ III weisen zusätzlich auch die unteren Inzisiven kariöse Läsionen auf (Abbildung 2.4). Grund für die Erkrankung sind in der Regel ebenfalls eine 
kariogene Ernährung und mangelnde Mundhygiene. Die Progredienz der Krankheit ist vielfach rasant (Wyne 1999).

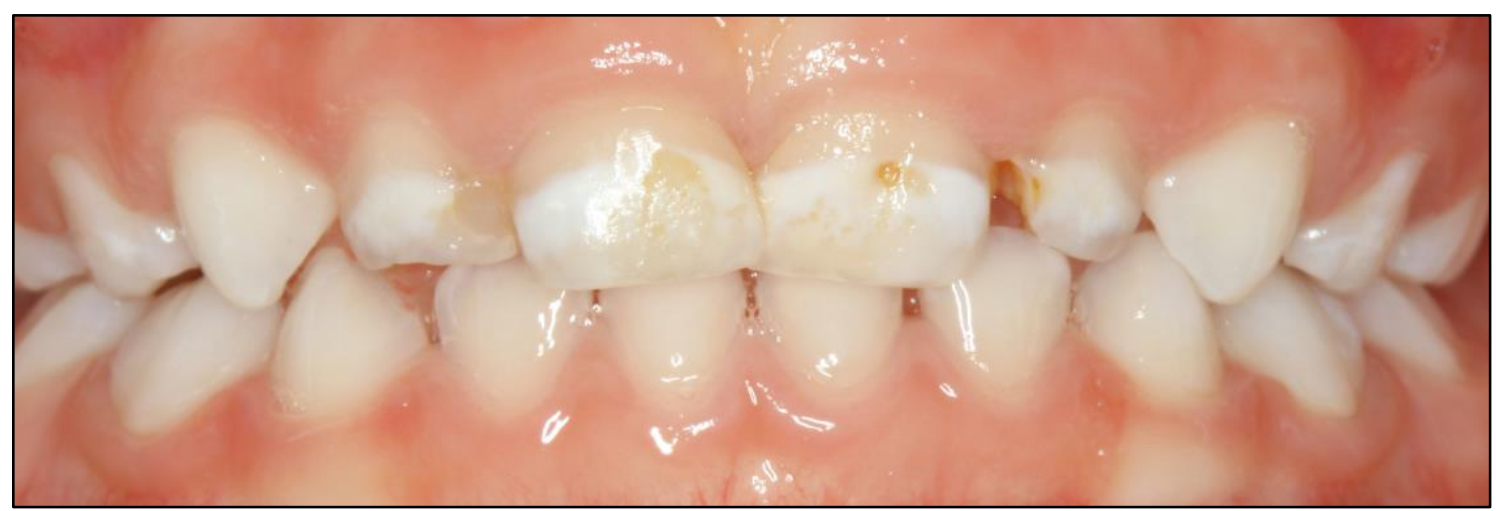

Abbildung 2.2: ECC-Typ I.

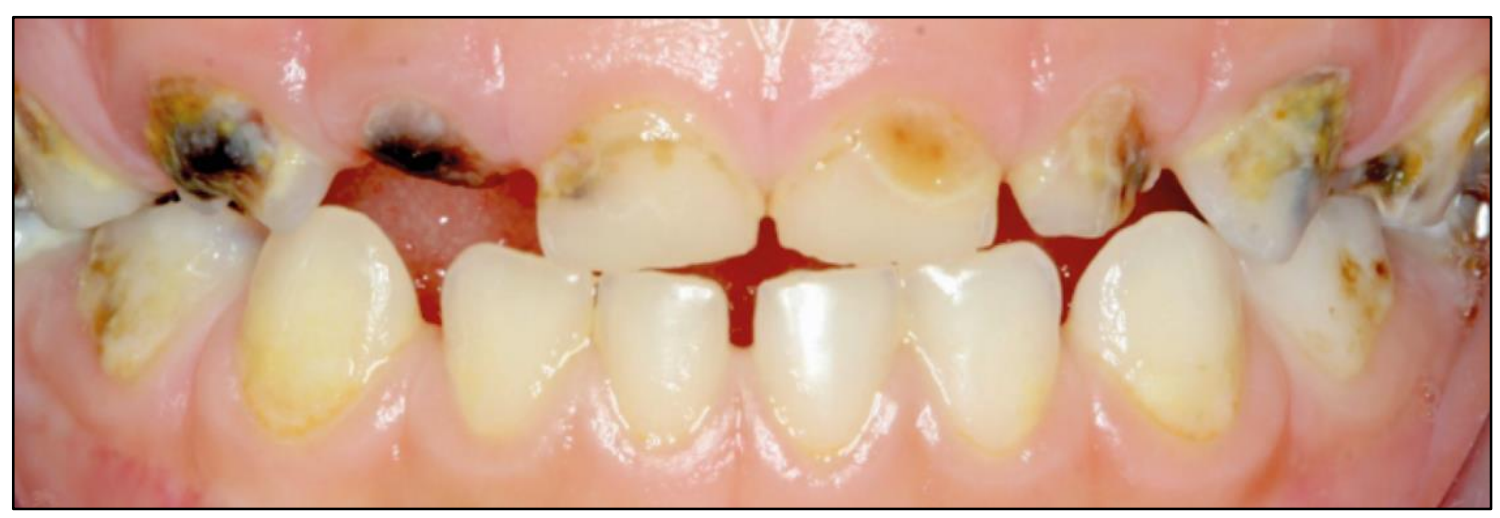

Abbildung 2.3: ECC-Typ II.

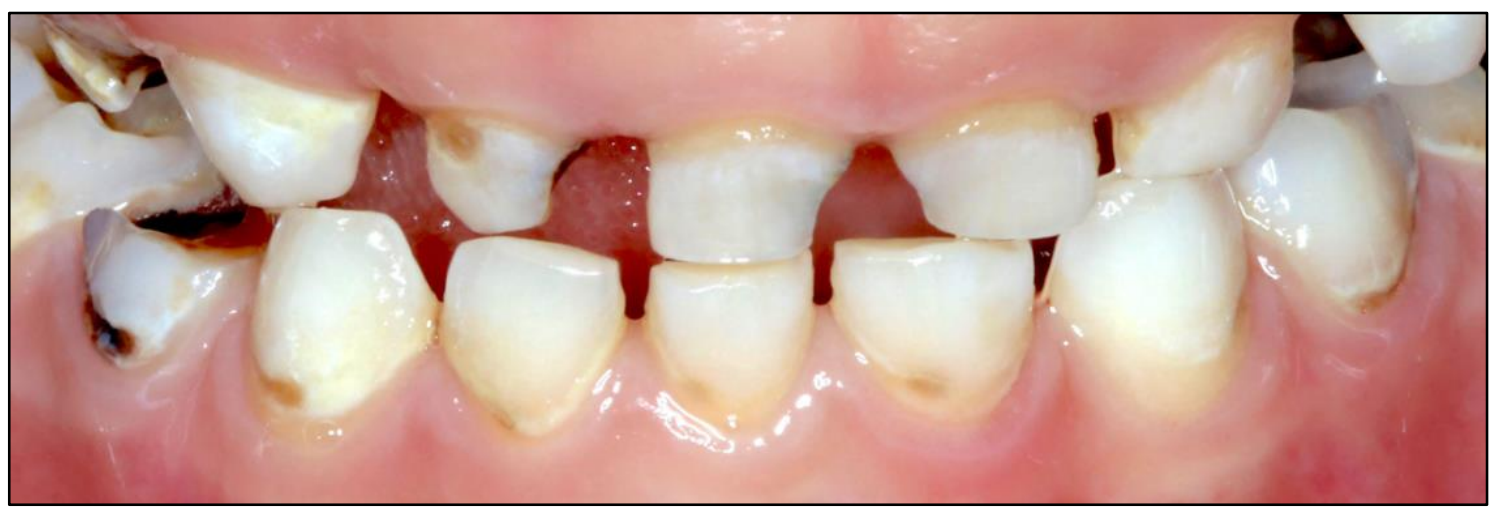

Abbildung 2.4: ECC-Typ III.

\subsubsection{Besonderheiten der Karies an Milchzähnen}

Die Grundsätze zur Entstehung von Karies gelten sowohl für die bleibenden Zähne als auch für die Milchzähne. Trotzdem gibt es Unterschiede zwischen beiden Dentitionen. Die Schmelzdicke der Milchzähne ist deutlich dünner als die der bleibenden Zähne (De Menezes Oliveira et al. 2009). Außerdem ist der 
Abstand zwischen Pulpa und Zahnoberfläche der juvenilen Zähne kleiner (Arnim und Doyle 1959; Stambaugh und Wittrock 1977). Histologisch liegen ebenfalls Differenzen vor: Die Dentinkanälchen der Milchzähne sind größer als die der bleibenden Zähne (Sumikawa et al. 1999), und die Mineralisation fällt geringer aus (Wilson und Beynon 1989; De Menezes Oliveira et al. 2009). In der chemischen Zusammensetzung unterscheiden sich Milch- und bleibende Zähne lediglich im zervikalen Bereich (Naujoks et al. 1967).

In-vitro-Studien konnten zeigen, dass eine Karies im Milchgebiss schneller voranschreitet als im bleibenden Gebiss (Featherstone und Mellberg 1981; Sønju Clasen et al. 1997; Ando et al. 2001). Zu gleichen Ergebnissen kamen klinische Verlaufsstudien, die die Progression von Schmelzkaries beider Dentitionen verglichen (Shwartz et al. 1984).

\subsubsection{Folgen von Karies bei Kindern}

Neben dem Zahnhartsubstanzverlust hat Karies noch andere weitreichende gesundheitliche Auswirkungen. Karies ist häufig mit Schmerzen verbunden (Agostini et al. 2001) und kann langfristig zur vollständigen Zerstörung der Zahnkrone führen (Robke 2008). Aufgrund der Substanzdefekte und den damit einhergehenden Schmerzen ist vielfach die Nahrungsaufnahme eingeschränkt (Naidu et al. 2016; BaniHani et al. 2018). Daraus kann ein Gewichtsverlust dieser Kinder resultieren (Acs et al. 1992). Bei ausgeprägten kariösen Läsionen kann es zudem zu apikalen Entzündungen und infolgedessen zu systemischen Bakteriämien kommen (Roberts und Holzel 2002). Insgesamt ist die Lebensqualität betroffener Patienten stark eingeschränkt, sie verbessert sich nach abgeschlossener und erfolgreicher Behandlung (Hirsch et al. 2000; Filstrup et al. 2003; BaniHani et al. 2018).

Therapieoptionen für Milchzahnkaries sind in der Regel Füllungen, Stahlkronen, Pulpotomien oder Extraktionen, wobei jede Behandlung individuell zu bewerten ist (Ritzmann-Fuchs und Krämer 2008). Eine besondere Gefahr, die von Milchzahnkaries ausgeht, ist die Beeinflussung der bleibenden Dentition. Ein frühzeitiger Milchzahnverlust oder eine Extraktion führen häufig zu kieferorthopädischen Fehlstellungen (Northway 2000; Robke 2008), apikale Entzündungen der Milchzähne zu Mineralisationsstörungen der bleibenden Zähne (Wetzel 1986). 


\subsubsection{Risikofaktoren und Prävention}

\subsubsection{Ernährung}

Wie wichtig die durch die Nahrung aufgenommenen Kohlenhydrate für die Entstehung von Karies sind, konnten viele Studien in der Vergangenheit nachweisen. So erhielten Heimkinder in der Hopewood-House-Studie nur Essen mit einem sehr niedrigen Anteil an niedermolekularen Zuckern, wodurch sie im Vergleich zu anderen Kindern mit gewöhnlichen Essgewohnheiten weniger kariöse Läsionen entwickelten (Lilienthal et al. 1953). Im Rahmen der Turku-Zuckerstudien erhielten die Probanden über Monate hinweg Mahlzeiten, die entweder mit Saccharose oder mit dem Zuckeraustauschstoff Xylit gesüßt wurden. Unter der Einnahme von Saccharose entwickelten sich neue kariöse Läsionen, was hingegen nicht unter der Einnahme von Xylit geschah (Scheinin und Mäkinen 1976).

Wichtig zu betonen ist, dass die Art des Zuckers nicht entscheidend ist, sowohl Monosaccharide als auch Disaccharide tragen in hohem Maße zur Entstehung und Progression von Karies bei (Shaw et al. 1967; Green und Hartles 1969). Relevant für die Kariesentstehung ist primär die Häufigkeit des Zuckerkonsums, was in der Vipeholm-Studie eindrucksvoll nachgewiesen werden konnte. Bei Probanden, die viele kleine Einheiten Zucker über den Tag verteilt zu sich nahmen, stieg die Anzahl an kariösen Läsionen stärker an als bei Probanden, die große Mengen Zucker während der Hauptmahlzeiten zu sich nahmen (Gustafsson et al. 1954). Für Kinder bedeutet dies, dass ihr Risiko für die Entstehung von Karies steigt, wenn sie häufig gesüßte Getränke oder Säfte trinken (Warren et al. 2008; Deichsel et al. 2012), ebenso, wenn sie vier oder mehr zuckerhaltige Zwischenmahlzeiten konsumieren (Holbrook et al. 1995). Stark gefährdet sind außerdem Kinder, die ihre Trinkflaschen mit zuckerhaltigen Getränken nachts mit ins Bett nehmen (Hallett und O'Rourke 2003; Bissar et al. 2014). Daher sollten Kinder nur zu den Hauptmahlzeiten süße Getränke erhalten und dazwischen ausschließlich Wasser oder ungesüßten Tee trinken. Diskutiert wird ferner ein Zusammenhang zwischen frühkindlicher Karies und Stillen. Einige Studien benennen einen negativen Einfluss der Stillauer (Hallett und O'Rourke 2003; Bissar et al. 2014), andere Autoren sehen ihn nur im nächtlichen Stillen (Pieper et al. 2012), weitere Untersuchungen fanden keinen 
Zusammenhang zwischen dem Stillen und der Entstehung von Karies (Deichsel et al. 2012).

\subsubsection{Mundhygiene}

Zähneputzen ist die einfachste und effizienteste Möglichkeit der oralen Biofilmkontrolle (Staehle et al. 2007), somit ist eine schlechte Mundhygiene als ein Risikofaktor für die Entstehung von Karies anzusehen (Alm et al. 2007; Menghini et al. 2008). Deswegen empfehlen Fachgesellschaften ab dem Durchbruch des ersten Zahnes mit dem Zähneputzen zu beginnen (Deutsche Gesellschaft für Zahn-, Mund- und Kieferheilkunde 2013; American Dental Association Council on Scientific Affairs 2014; American Academy of Pediatric Dentistry 2016). Um Kinder an das regelmäßige, tägliche Zähneputzen heranzuführen, sollten sie mit eineinhalb Jahren mit dem selbstständigen Zähneputzen beginnen (Ogasawara et al. 1992). Die motorischen Fähigkeiten entwickeln sich erst mit zunehmendem Alter, sodass, je nach motorischer Entwicklung, bis mindestens zur dritten Klasse durch erwachsene Bezugspersonen nachgeputzt werden sollte (Zwiauer et al. 2011; Tschoppe et al. 2012; Deutsche Arbeitsgemeinschaft für Jugendzahnpflege e. V. 2016).

\subsubsection{Fluorid}

Eine tragende Rolle in der Kariesprävention spielen Fluoride. Dabei handelt es sich um Verbindungen des Elementes Fluor. Sie kommen natürlicherweise im Trinkwasser und in Lebensmitteln vor (Smith und Ekstrand 1996) und können sich anstelle eines herausgelösten Hydroxydions im Zahnschmelz anlagern, wodurch Fluorapatid entsteht (Fejerskov et al. 2015). Fluorapatit weist im Vergleich zu Hydroxylapatit eine deutlich geringere Löslichkeit auf (McCann 1968; Brown et al. 1977). Die geringere Löslichkeit bleibt auch bei niedrigen $\mathrm{pH}-$ Werten erhalten und schützt auf diese Weise vor den sauren Stoffwechselprodukten der Mikroorgansimen (Christoffersen et al. 1984; ten Cate und Featherstone 1991).

Es gibt zwei verschiedene Wege, wie eine kariesprotektive Wirkung mit Hilfe von Fluoriden erreicht wird: zum einen präeruptiv und zum anderen posteruptiv. Die präeruptive Anwendung zielt auf die Schmelzbildung ab. Über den Gastrointestinaltrakt aufgenommenes Fluorid steht während der 
Schmelzbildung zur Verfügung und wird vermehrt in das Apatitgitter des Schmelzes eingebaut (Jay 1946). Frühe Studien konnten zeigen, dass Kinder, die in Regionen mit einem hohen Fluoridgehalt des Trinkwassers aufwuchsen, weniger kariöse Läsionen entwickelten als Kinder, die mit einem niedrigen Fluoridgehalt im Trinkwasser aufwuchsen (Dean et al. 1941; Dean et al. 1942). Bei Personen, die während der Schmelzbildung systemisch Fluorid (1,4 ppm im Trinkwasser) aufnahmen, zeigten jedoch histologische Untersuchungen der Zähne, dass der Anteil des Fluorids in der oberflächlichen Schmelzschicht nicht erhöht war (Kidd et al. 1980). Die protektive Wirkung wurde daher auf die lokale Einwirkung beim Trinken zurückgeführt, sodass die Theorie der posteruptiven Schmelzreifung entwickelt wurde. Diese Theorie begründet die Effektivität des Fluorids damit, dass die zugeführten Fluoridionen die Hydroxydionen des Hydroxylapatites an der Schmelzoberfläche ersetzen und Fluorapatit entsteht (Knappwost 1951; Knappwost 1956; Lin et al. 1981; de Leeuw 2004). Zudem können sich die Fluoridionen auch mit Kalziumionen zu Kalziumfluorid verbinden, welches als Speicher des Fluorids im Speichel dient (Larsen und Bruun 1986) und somit noch mehrere Stunden im Mund zur Verfügung steht (Lambrou et al. 1981). Das Kalziumfluorid kann sich als Niederschlag am Schmelz abscheiden und dort, bei niedrigen $\mathrm{pH}$-Werten, vor Demineralisation schützen (Wong et al. 1987).

Heute wird der posteruptive Mechanismus als Hauptgrund für die präventive Wirkung von Fluoriden gegen Karies angesehen (Thylstrup 1990; Fejerskov 2004). Da die deutsche Trinkwasserverordnung eine maximal zulässige Höchstkonzentration von Fluorid im Trinkwasser von $1,5 \mathrm{ppm}$ vorsieht (Verordnung über die Qualität von Wasser für den menschlichen Gebrauch (Trinkwasserverordnung - TrinkwV) 2018) und es in Deutschland verboten ist, dem Trinkwasser Fluorid zu zusetzen (Umweltbundesamt 2017), werden heute für die Kariesprävention überwiegend fluoridhaltige Zahnpasten eingesetzt. Ab dem Durchbruch des ersten Zahnes sollten die Zähne einmal am Tag mit Kinderzahnpasta (500 ppm Fluorid) geputzt werden, ab dem Alter von zwei Jahren zweimal täglich mit Kinderzahnpasta und ab dem Durchbruch des ersten bleibenden Zahnes zweimal täglich mit Erwachsenenzahnpasta (1.000-1.500 ppm Fluorid) (Deutsche Gesellschaft für Zahn-, Mund- und Kieferheilkunde 2013). 


\subsubsection{Soziodemographischen Faktoren}

Neben den bereits genannten Gründen für die Entstehung von Karies gibt es auch soziodemographische Faktoren, die das Risiko für eine Karies bei Kindern begünstigen. Dazu zählen vor allem ein niedriger Sozialstatus, ein niedriges Einkommen sowie ein niedriges Bildungsniveau der Eltern (Borutta et al. 2002; Schwendicke et al. 2015; Wagner und Heinrich-Weltzien 2016). Ebenso weisen Kinder mit Migrationshintergrund häufiger kariöse Läsionen auf als Kinder ohne Migrationshintergrund (Menghini et al. 2008; Bissar et al. 2014). Einige Studien fanden zudem einen Zusammenhang zwischen Karies und dem Geschlecht der Kinder, so waren männliche Patienten in einigen Studien häufiger betroffen als weibliche (Willerhausen et al. 2007; Menghini et al. 2008).

\subsubsection{Epidemiologie von Karies bei Kindern in Deutschland}

Systematische Untersuchungen konnten zeigen, dass sich dank verbesserter Prophylaxekonzepte die Mundgesundheit von Kindern in Deutschland in den letzten Jahrzehnten deutlich verbessert hat. So sank beispielsweise im Bundesdurchschnitt bei den 6-7-Jährigen die Kariesprävalenz zwischen 1994 und 2009 um 43,5 \% (Pieper 2010). In den letzten Jahren ist die Reduktion der Kariesprävalenz jedoch nicht mehr so ausgeprägt gewesen (Deutsche Arbeitsgemeinschaft für Jugendzahnpflege e.V. 2017). Im Bundesland Niedersachsen zeigt sich ein ähnlicher Trend: der Anteil der naturgesunden Milchgebisse der 6-7-Jährigen stieg von 48,8 \% im Jahr 2000 (Pieper 2001) auf $56,8 \%$ im Jahr 2016 (Deutsche Arbeitsgemeinschaft für Jugendzahnpflege e. V. 2017). Das bedeutet trotz allem, dass immer noch $28,3 \%$ der Kinder in Niedersachsen im Milchgebiss unbehandelte kariöse Läsionen aufweisen (Deutsche Arbeitsgemeinschaft für Jugendzahnpflege e. V. 2017). Auch die Karieserfahrung der 12-Jährigen nimmt in Deutschland immer weiter ab (Schiffner 2016).

\subsubsection{Soziale und sozioökonomische Folgen}

Zusätzlich zu den gesundheitlichen Folgen der Karies gibt es auch gravierende sozioökonomische Auswirkungen. In der sozialen Interaktion mit anderen Menschen wird eine sichtbare Karies bei Erwachsenen vom Gegenüber als weniger attraktiv beurteilt (Karunakaran et al. 2011). Die Auswirkungen 
sichtbarer Karies zeigen sich ebenfalls in der Beurteilung von Sozialkompetenz und Intelligenz. Personen mit sichtbaren kariösen Läsionen wurden als weniger sozialkompetent und weniger intelligent eingeschätzt als Personen ohne sichtbare Defekte an den Zähnen (Somani et al. 2010). Gleiches gilt, wenn Kinder die Fotos von anderen Kindern mit auffälligen dentalen Befunden beurteilen. Die Bewertung der Kinder geht sogar so weit, dass fehlende Frontzähne als Zeichen von Aggressivität gewertet werden (Shaw 1981). Betroffene Kinder mit einer frühkindlichen Karies geben an, dass sie wegen ihrer Zähne bereits von andere Kinder gehänselt wurden (Filstrup et al. 2003).

In Studien zur Wahrnehmung der dentalen Ästhetik stellte sich allerdings heraus, dass eine dentale Veränderung für Laien deutlich stärker ausgeprägt sein muss als für Zahnärzte, um als ästhetisch störend bewertet zu werden (Kokich et al. 1999; Kokich et al. 2006).

Neben den sozialen Aspekten von Karies bei Kindern gibt es auch ökonomische Folgen. Die Behandlungskosten von frühkindlicher Karies sind um ein Vielfaches höher als Präventionskosten und damit die Belastungen für das Gesundheitssystem. Je früher mit der Prävention begonnen wird, desto geringer ist die Wahrscheinlichkeit für das Auftreten von Karies, respektive den daraus resultierenden Behandlungskosten (Casamassimo et al. 2009; Nowak et al. 2014; O'Neill et al. 2017). 


\section{Ziele der Arbeit}

Bisherige Eye-Tracking-Studien untersuchten die Wahrnehmung von Gesichtsasymmetrien und konnten nachweisen, dass asymmetrische Gesichtsregionen länger betrachtet wurden als die gleiche Gesichtsregion bei Patienten ohne Asymmetrien (Meyer-Marcotty et al. 2010b; Litschel et al. 2015; van Schijndel et al. 2015). Eine Studie verglich die Wahrnehmung zwischen Laien und Experten und konnte zeigen, dass Experten die Oberlippe von Patienten mit Lippen-Kiefer-Gaumen-Spalten sowohl initial schneller als auch insgesamt länger und häufiger betrachteten als Laien (Dindaroğlu et al. 2017). Auch wurden die Auswirkungen verschiedener dentaler Befunde wie beispielsweise fehlende Zähne, sichtbare Kronen oder Karies auf die Bewertung der Gesichtsattraktivität erforscht. Diese Untersuchungen konnten nachweisen, dass Gesichter mit sichtbaren kariösen Zähnen als unattraktiver empfunden werden und die Erkrankung soziale Ablehnung hervorruft (Shaw 1981; Filstrup et al. 2003; Kershaw et al. 2008; Karunakaran et al. 2011). Weitere Attraktivitätsstudien konnten zeigen, dass Experten bei Zahnfehlstellungen insgesamt strenger in der Beurteilung von Abweichungen sind als Laien (Kokich et al. 1999; Kokich et al. 2006). Aktuell gibt es keine Studien, welche die Wahrnehmung von kariösen Zähnen insbesondere bei Kindern untersucht haben.

Das Ziel dieser Studie war es mittels einer Eye-Tracking-Untersuchung und Fragebögen zu ermitteln, ob verschiedene sichtbare Zahnbefunde (gesundes Gebiss, Karies und Zahnsanierung, z. B. Zahnfüllungen oder -extraktionen) bei Kindern das Blickverhalten von Erwachsenen beeinflussen und ob sich dies in einer anschließenden Bewertung hinsichtlich Valenz, Arousal und Attraktivität widerspiegelt, auch wenn Zahnveränderungen nur geringe optische Veränderungen darstellen. Darüber hinausgehend sollte untersucht werden, ob sich die visuelle Wahrnehmung und Bewertung von zahnmedizinischen Laien und Experten unterscheidet. Aufgrund der angeführten Literatur werden für die vorliegende Studie acht Hypothesen aufgestellt. 


\subsection{Hypothesen}

\subsubsection{Hypothese 1}

Als Nullhypothese wird aufgestellt, dass sich die initiale Aufmerksamkeit (erste Fixation) bei der Betrachtung von Kindergesichtern durch zahnmedizinische Laien und Experten nicht unterscheidet.

Als Alternativhypothese leitet sich ab, dass sich die initiale Aufmerksamkeit bei der Betrachtung von Kindergesichtern durch zahnmedizinische Laien und Experten unterscheidet.

\subsubsection{Hypothese 2}

Die Nullhypothese lautet, dass sich das Betrachtungsverhalten von Laien und Experten während der ersten beiden Sekunden nicht unterscheidet.

Als Alternativhypothese wird gebildet, dass sich das Betrachtungsverhalten von Laien und Experten während der ersten beiden Sekunden unterscheidet.

\subsubsection{Hypothese 3}

Als Nullhypothese wird formuliert, dass die Betrachtung von Kindergesichtern während der dritten Sekunde durch Laien und Experten sich nicht unterscheidet.

Die Alternativhypothese lautet, dass sich die Betrachtung von Kindergesichtern während der dritten Sekunde durch Laien und Experten unterscheidet.

\subsubsection{Hypothese 4}

Die Nullhypothese besagt, dass die initiale Betrachtung von Kindergesichtern nicht vom dentalen Befund (gesund, Karies und Zahnsanierung) abhängt.

Als Alternativhypothese ergibt sich, dass die initiale Betrachtung von Kindergesichtern vom dentalen Befund abhängt. 


\subsubsection{Hypothese 5}

Dass die Wahrnehmung von Kindergesichtern während der ersten beiden Sekunden nicht vom dentalen Befund abhängt, wird als Nullhypothese aufgestellt.

Die Alternativhypothese sagt aus, dass die Wahrnehmung von Kindergesichtern während der ersten beiden Sekunden vom dentalen Befund abhängt.

\subsubsection{Hypothese 6}

Als Nullhypothese wird formuliert, dass die Wahrnehmung von Kindergesichtern während der dritten Sekunde nicht vom dentalen Befund abhängt.

Die Alternativhypothese lautet, dass die Wahrnehmung von Kindergesichtern während der dritten Sekunde vom dentalen Befund ab hängt.

\subsubsection{Hypothese 7}

Dass Laien und Experten Kindergesichter nach Valenz, Arousal und Attraktivität nicht unterschiedlich beurteilen, besagt die Nullhypothese.

Als Alternativhypothese resultiert, dass Laien und Experten Kindergesichter nach Valenz, Arousal und Attraktivität unterschiedlich beurteilen.

\subsubsection{Hypothese 8}

Als Nullhypothese wird hergeleitet, dass dentale Befunde keinen Einfluss auf die Bewertung von Kindergesichtern nach Valenz, Arousal und Attraktivität haben.

Als Alternativhypothese folgt, dass dentale Befunde Einfluss auf die Bewertung von Kindergesichtern nach Valenz, Arousal und Attraktivität haben. 


\section{Material und Methoden}

Die vorliegende Studie wurde von der Ethik-Kommission der Universitätsmedizin Göttingen (Antragsnummer 36/2/16) genehmigt. Ab April 2016 erfolgten die Rekrutierung der Fotomodelle, die Aufnahme der Fotos sowie die Auswahl der Bilder an der Universitätsmedizin Göttingen. Die Eye-Tracking-Untersuchungen wurden zwischen Oktober 2016 und Februar 2017 am Institut für Psychologie der Stiftung Universität Hildesheim durchgeführt.

\subsection{Auswahl der Fotomodelle}

Die Fotomodelle wurden aus den ambulanten Patienten der Poliklinik für Präventive Zahnmedizin, Parodontologie und Kariologie und der Poliklinik für Kieferorthopädie der Universitätsmedizin Göttingen rekrutiert. Voraussetzungen für die Teilnahme an der Studie waren die Zustimmung des Kindes und der Erziehungsberechtigten oder der gesetzlichen Betreuer. Eingeschlossen wurden Kinder zwischen zwei und zehn Jahren, die keine Allgemeinerkrankungen, Verletzungen oder Verletzungsfolgen aufwiesen, die mit knöchernen und/oder weichgewebigen Veränderungen im Kopfbereich einhergingen.

\subsection{Fotoaufnahmen}

Die Fotoaufnahmen wurden mit einer Spiegelreflexkamera (Canon EOS 500D, Canon Inc., Tokyo, Japan) mit Makro-Objektiv (Canon EF 100 mm f/2.8 Macro USM, Canon Inc., Tokyo, Japan) und Aufsteckblitz (Canon Speedlite 580EX II, Canon Inc., Tokyo, Japan) erstellt. Die Aufnahmen erfolgten im Modus „Manuelle Belichtung“ bei einer Belichtungszeit von 1/200 s, einer Blende von f/8.0 und einer ISO-Empfindlichkeit von 200. Die Auflösung der Fotos betrug 4752 × 3168 px. Der Blitz wurde im Modus ETTL senkrecht auf das Gesicht der Patienten projiziert.

Von jedem Patienten wurden zwei verschiedene Fotos angefertigt: (1) neutral (Mund geschlossen, kein Lächeln) und (2) emotional (möglichst sichtbare Frontzähne, Lächeln). Um eine gute Vergleichbarkeit der Bilder zu erzielen, 
erfolgten die Aufnahmen standardisiert bei gleichen Lichtverhältnissen, gleicher Kamera-Gesicht-Positionierung und ohne möglicherweise ablenkende Details wie Schmuck oder Brille. Lange Haare wurden mithilfe von Haargummis oder Haarreifen zurückgehalten.

\subsection{Bearbeitung der Bilder}

Die Nachbearbeitung der Bilder erfolgte mit der Software Adobe Photoshop CC 2015 (Version 2015.0.0, Adobe Systems Inc., San José, CA, USA). Ziel der Bearbeitung war die Reduktion des Bildes auf das Gesicht. Die Kaschierung von Ohren, Haaren und des Hintergrundes erfolgte mit einem mittleren Grau (RGB 128, 128, 128), sodass nur die Gesichtsregionen Stirn, Augen, Nase, Mund und Kinn unberührt blieben.

Anschließend wurden die Fotos mittels Adobe InDesign CC (Version 2015.0, Adobe Systems Inc., San José, CA, USA) so ausgerichtet, dass alle Bilder einen identischen Pupillenabstand aufwiesen. Der Pupillenmittelpunkt des rechten Auges wurde ausgehend von der oberen linken Ecke auf die Position $120 \times 240$ und der Pupillenmittelpunkt des linken Auges auf die Position $280 \times 240$ eingestellt. Die Gesichtsmittellinie wurde zentral zum Bild ausgerichtet. Die Bildgröße wurde auf $400 \times 600$ px angepasst.

\subsection{Auswahl der Bilder}

\subsubsection{Fragebogen zu den Bildern}

Die Bewertung der Bilder erfolgte sowohl im Vorauswahlverfahren als auch im Rahmen der Eye-Tracking-Untersuchung mit Self-Assessment Manikins (SAM) in der 9-Punkte-Likert-Form von Irtel (2007) zur Erfassung des subjektiven Gefühlszustandes der emotionalen Qualitäten Arousal (Erregung / Aktiviertheit) und Valenz (Vergnügen / Wohlgefallen) (Bradley und Lang 1994). Die Bewertung der Attraktivität erfolgte analog mit einer 9-stufigen Skala ( 1 = unattraktiv, 5 = durchschnittliches Aussehen, 9 = attraktiv). 


\subsubsection{Vorauswahl der Fotos nach emotionalen Gesichtspunkten}

Die Vorauswahl der Bilder nach emotionalen Gesichtspunkten erfolgte anhand der bearbeiteten Fotos mit dem neutralen Gesichtsausdruck. Ziel war es, für die Eye-Tracking-Untersuchung ausschließlich die Kinder auszuwählen, deren neutraler Gesichtsausdruck auch als neutral wahrgenommen wird. Um Verzerrungen in der Eye-Tracking-Studie zu vermeiden, wurden Bilder, die beim Betrachter (starke) emotionale Gefühle hervorriefen oder als besonders attraktiv oder unattraktiv bewertet wurden, in diesem Schritt ausgeschlossen.

Von den insgesamt 124 fotografierten Kindern wurden 35 Patienten ausgeschlossen, weil die Fotos der Kinder unscharf, verwackelt oder aufgrund unterschiedlicher Perspektiven nicht vergleichbar waren. Besonders Kleinkinder konnten für die Fotoaufnahmen häufig nicht ausreichend ruhig oder gerade sitzen.

Die Bewertung der verbliebenen 89 Patienten nach Valenz, Arousal und Attraktivität wurde durch 26 Zahnärztinnen und Zahnärzte der Poliklinik für Präventive Zahnmedizin, Parodontologie und Kariologie Göttingen (Alter in Jahren: $33,5 \pm 11,4)$ mit Hilfe des oben beschriebenen Fragebogens vorgenommen. Die Analyse der Bewertungen wurde mittels Kruskal-Wallis-Tests mit Post-hoc-Testung nach Nemenyi durchgeführt. Weiterhin wurden nur die Bilder eingeschlossen, die sich innerhalb der drei Frageparameter (Valenz, Arousal, Attraktivität) nicht signifikant unterschieden. Als Signifikanzniveau wurde $\alpha=0,001$ definiert. Nach emotionalen Gesichtspunkten konnten somit 54 Bildpaare zur zahnmedizinischen Einordnung zugelassen werden.

\subsubsection{Zahnmedizinische Einordnung}

Die zahnmedizinische Einordnung der Bilder in die Klassifizierungen (1) "gesund“, (2) „Karies“ und (3) „Zahnsanierung“ erfolgte durch 15 erfahrene Zahnärztinnen und Zahnärzte der Poliklinik für Präventive Zahnmedizin, Parodontologie und Kariologie Göttingen (Alter in Jahren 31,0 99,7). Dazu wurden die 54 Fotos der emotionalen Bilder bewertet, die nach der Vorauswahl noch eingeschlossen waren. Als Zahnsanierung zählten Füllungen, Stahlkronen und/oder Lücken nach Zahnextraktionen. Wenn die Interrater-Übereinstimmung 
durch die Zahnärzte kleiner als $60 \%$ war, wurden die Bildpaare ausgeschlossen. Um darüber hinaus eine gleichmäßige Verteilung an Fotos in den Klassifizierungsgruppen zu erreichen, wurden weitere 15 Patienten ausgeschlossen. Von den final eingeschlossenen 39 Bildern fielen 13 in die Kategorie "gesund“, 13 in die Kategorie „Karies“ und 13 in die Kategorie „Zahnsanierung“. Das Flussdiagramm zur Auswahl und Verteilung der Fotos auf die unterschiedlichen Gruppen ist in Abbildung 4.1 dargestellt. Die Alters-, Geschlechts- und Gebissverteilung ist in Tabelle 4.1 zusammengefasst dargestellt.

\section{Fotografierte Patienten \\ $\mathrm{n}=124$}

Ausschluss: unzureichende Vergleichbarkeit der Bilder

$\mathrm{n}=35$

Vorauswahl (Valenz, Arousal \& Attraktivität)

$\mathrm{n}=89$

$\mathrm{n}=35$

\section{Zahnmedizinische Einordnung}

$\mathrm{n}=54$

Ausschluss: Interrater Übereinstimmung $<60 \%$, gleichmäßige Gruppengröße $\mathrm{n}=15$

Final eingeschlossene Bildpaare

$\mathrm{n}=39$

Gesund
$n=13$


Tabelle 4.1: Verteilung der Bildpaare der Patienten hinsichtlich der verschiedenen Klassifizierungen (SD: Standardabweichung).

\begin{tabular}{lccccccc}
\hline \multirow{2}{*}{$\begin{array}{c}\text { Gebiss- } \\
\text { Klassifizierung }\end{array}$} & Anzahl & \multicolumn{2}{c}{ Alter } & \multicolumn{2}{c}{ Geschlecht } & \multicolumn{3}{c}{ Gebiss } \\
\cline { 3 - 7 } & & Mittelwert \pm SD & männlich & weiblich & Milch- & Wechsel- & Bleibendes \\
\hline Gesund & 13 & $6,4 \pm 2,3$ & 7 & 6 & 5 & 7 & 1 \\
Karies & 13 & $6,0 \pm 2,4$ & 6 & 7 & 7 & 6 & 0 \\
Zahnsanierung & 13 & $6,9 \pm 1,8$ & 4 & 9 & 10 & 2 & 1 \\
\hline
\end{tabular}

\subsection{Eye-Tracking-Untersuchung}

Die Eye-Tracking-Untersuchung fand am Institut für Psychologie der Stiftung Universität Hildesheim in Zusammenarbeit mit der Arbeitsgruppe Neurodidaktik unter der Leitung von Prof. Dr. rer. nat. Kristian Folta-Schoofs statt.

\subsubsection{Probandinnen}

Als Probandinnen wurden Zahnmedizinstudierende der Universität Göttingen und Studierende der Psychologie der Stiftung Universität Hildesheim rekrutiert.

\subsubsection{Einschlusskriterien}

Eingeschlossen wurden gesunde, weibliche Studierende der Universitäten Göttingen (Zahnmedizin) und Hildesheim (Psychologie), die Rechtshänder waren und deren schriftliches Einverständnis zur Studienteilnahme vorlag. Die Zahnmedizinstudierenden mussten sich im klinischen Studienabschnitt befinden. Diese werden als (zahnmedizinische) Experten bezeichnet, weil sie bereits in der Diagnostik und Therapie von Karies geschult waren. Die Studierenden der Psychologie wiesen in dieser Hinsicht keine Vorbildung auf und können daher als (zahnmedizinische) Laien angesehen werden.

\subsubsection{Ausschlusskriterien}

Folgende Ausschlusskriterien zur Teilnahme an der Studie wurden mittels eines Fragebogens erhoben (Abbildung 10.1):

a) Fehlendes Einverständnis zur Teilnahme an der Studie,

b) Fehlende Kooperation, 
c) Alter $<18$ Jahre,

d) Linkshänder,

e) Brillenträger,

f) Personen mit schweren Allgemeinerkrankungen, insbesondere Zustände, die mit vorübergehenden oder dauerhaften Einschränkungen des Immunstatus einhergehen,

g) Personen mit schweren psychischen Erkrankungen, die mit einer veränderten Wahrnehmung oder neurophysiologischen Reizverarbeitung einhergehen,

h) Personen, die regelmäßig oder zum Zeitpunkt der Untersuchung Drogen oder Medikamente einnehmen, die mit einer veränderten Wahrnehmung oder neurophysiologischen Reizverarbeitung einhergehen.

\subsubsection{Versuchsaufbau}

Der technische Aufbau der Versuche fand in Anlehnung an vorhergegangene Studien statt (Folta und Mähler 2010; Folta-Schoofs et al. 2015). Die Untersuchungen wurden in einer akustisch abgeschirmten Box $(2,0 \mathrm{~m} \times 4,0 \mathrm{~m} \times 2,5 \mathrm{~m})$ mit einer auf die Helligkeit des Bildschirms abgestimmten Beleuchtung von ca. $50 \mathrm{~cd} / \mathrm{m}^{2}$ Leuchtdichte durchgeführt.

Als Präsentationsbildschirm diente ein QUATO ${ }^{\circledR}$ Intelli Proof 240 LED excellence 24" LED-TFT (Auflösung 1920 x 1200 px, Bildfrequenz $60 \mathrm{~Hz}$, Quatographic Technology $\mathrm{GmbH}$, Braunschweig), das entspricht einer Full-HD Auflösung. Der Analysebereich lag mittig auf dem Bildschirm und hatte eine Auflösung von $1280 \times 1024$ px. Mit Hilfe des Eye-Tracking-Systems iView $X^{\mathrm{TM}}$ Hi-Speed 1250 (SensoMotoric Instruments $\mathrm{GmbH}$, Teltow) und der Software iView $X^{\mathrm{TM}}$ (Version 2.7 built 13, SensoMotoric Instruments $\mathrm{GmbH}$, Teltow und Intel ${ }^{\circledR}$ Core $^{\mathrm{TM}} 2$ Duo 2,66 GHz, Betriebssystem Windows XP Professional Service Pack 3) wurden die Bewegungen des linken Auges mit einer Frequenz von $1250 \mathrm{~Hz}$ aufgezeichnet. Die Versuchssteuerung und Stimulationskontrolle erfolgte mithilfe eines Apple iMac 24" (Prozessor $3.06 \mathrm{GHz}$ Intel ${ }^{\circledR}$ Core 2 Duo, Betriebssystem Mac OS X 10.5.8, Apple Inc., Cupertino, CA, USA) durch das Programm MatLab (Version 7.8.0.0347, R2009a, The MathWorks Inc., Natick, MA, USA) und der Erweiterung Psychophysics Toolbox Version 3.0.9 (Brainard 
1997; Pelli 1997; Kleiner et al. 2007). Über ein internes Netzwerk standen das Eye-Tracking-System und das Programm MatLab in Verbindung.

\subsubsection{Versuchsablauf}

Vor Beginn der Eye-Tracking-Untersuchung erfolgten eine ausführliche Aufklärung der Probandinnen über den Ablauf und eine schriftliche Einwilligung zur Studienteilnahme. Die genauen Studienziele wurden den Probandinnen erst nach Durchführung der Experimente erläutert. Die Ein- und Ausschlusskriterien wurden mithilfe eines Fragebogens erfasst (Abbildung 10.1). Zur Bestimmung der Händigkeit diente der Edinburgh Handedness Inventory Fragebogen, der mit zehn Fragen die Bevorzugung der Hände und mit je einer Frage das bevorzugten Auge und den bevorzugten Fuß abfragt (Oldfield 1971). Anschließend kann ein Händigkeitsquotient ermittelt werden, dieser kann Werte von +100 bis -100 ergeben. Ein Wert über +40 bedeutet Rechtshändigkeit, Werte zwischen +40 und -40 Beidhändigkeit und Werte unter - 40 Linkshändigkeit des Probanden (Robinson 2013).

Die Aufgabenstellung wurde standardisiert anhand einer schriftlichen Anweisung vorgegeben. Vor Beginn des Experiments wurde für jede Probandin eine 9-Punkt-Kalibration des Eye-Tracking-Systems durchgeführt. Die Probandinnen saßen während der Aufzeichnungen mit einem Sehabstand von $100 \mathrm{~cm}$ vor dem Präsentationsbildschirm. Um eine ruhige Sitzposition zu gewährleisten, wurde das Kinn auf eine Kinnstütze und die Stirn an eine Stirnplatte gelegt.

Bei der Eye-Tracking-Untersuchung wurden die insgesamt 39 vorausgewählten Fotopaare von 13 Kindern mit gesunden Zähnen, 13 Kindern mit kariösen Zähnen und 13 Kindern mit Zahnsanierung präsentiert. Dabei wurden jeweils das neutrale und das emotionale Foto eines Kindes nebeneinander auf dem Monitor dargestellt. Von jedem der 39 Fotopaare wurden zwei verschiedene Präsentationsbildpaare gezeigt, auf dem einen war das neutrale Foto links und das emotionale Foto rechts und auf dem anderen Präsentationsbildpaar war das emotionale Foto links und das neutrale Foto rechts angeordnet. Die Bilder wiesen dabei eine Auflösung von 400 px × 600 px auf. Der Abstand der Bilder zueinander betrug $460 \mathrm{px}$. Bei einem Abstand der Probandinnen von $100 \mathrm{~cm}$ zum Bildschirm ergab sich somit ein Sehwinkel von $7,11^{\circ}$ vom linken zum 
rechten Foto. Der Hintergrund des Bildschirms war auf die Hintergründe der Fotos abgestimmt worden und zeigte ebenfalls ein mittleres Grau.

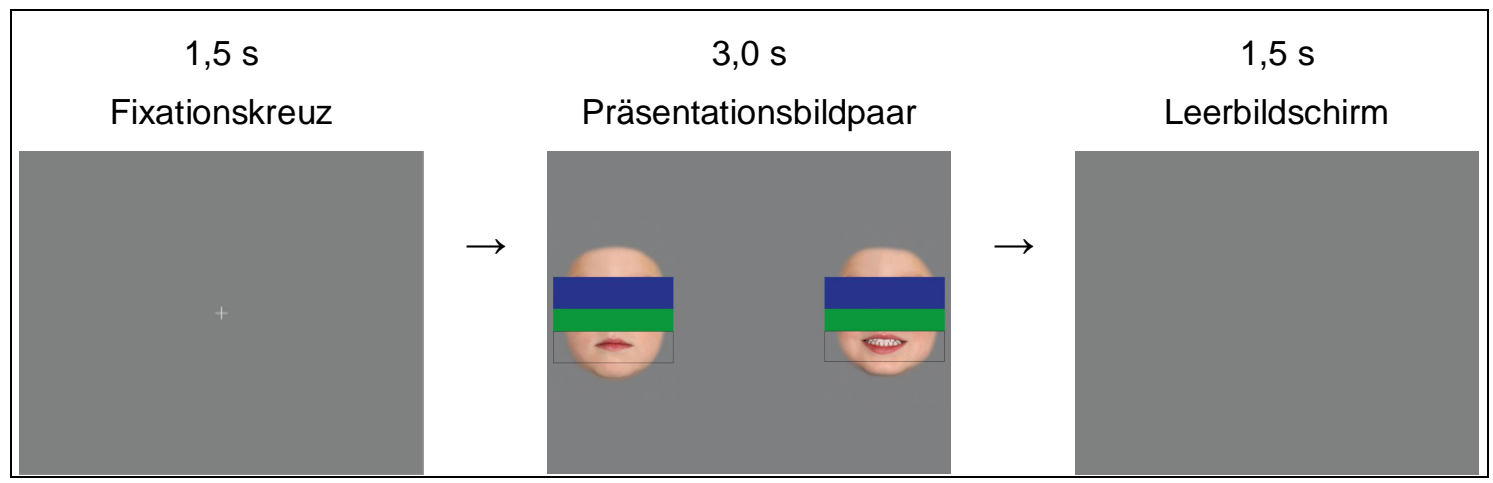

Abbildung 4.2: Ablauf des Präsentationsbildschirms. Augen und Nase sind aus datenschutzrechtlichen Gründen unkenntlich gemacht.

Zu Beginn jedes Bildablaufes wurde für 1,5 Sekunden zentral auf dem Bildschirm ein weißes Fixationskreuz dargestellt. Die Präsentation der Bildpaare erfolgte für 3,0 Sekunden. Zur Entspannung der Augen und zur Unterbrechung der Reizsetzung wurde im Anschluss für 1,5 Sekunden ein grauer Leerbildschirm angezeigt, bevor automatisch die Präsentation des nächsten Bildpaares begann (Abbildung 4.2). Während des Experimentes wurde durch den Versuchsleiter über einen Kontrollmonitor eine dauerhafte Fixationskontrolle durchgeführt und das Betrachtungsverhalten, insbesondere die Fixation des weißen Fixationskreuzes, vor dem nächsten Prästationsbild überwacht. Die Reihenfolge der Präsentationsbilder erfolgte randomisiert entsprechend der Programmierung von MatLab mit Hilfe der Erweiterung Psychophysics Toolbox Version 3.0.9 (Brainard 1997; Pelli 1997; Kleiner et al. 2007). Die gesamte Untersuchung mit allen 78 Präsentationsbildern dauerte 7,8 Minuten.

Im Anschluss an die Eye-Tracking-Untersuchung wurden den Probandinnen im Rahmen einer Bewertungsrunde erneut alle 78 Bilder gezeigt. Die Fotos wurden den Probandinnen dafür als hochwertig gedruckte Fotos vorgelegt (Auflösung: 300 dpi, Größe: DIN A4). Die Bewertungen erfolgten mit denselben Fragen, die zur Vorauswahl der Fotos genutzt wurden. Zuerst wurden die Fragen zu Valenz, Arousal und Attraktivität zu den neutralen Fotos und im Anschluss daran dieselben Fragen zu den emotionalen Fotos beantwortet. Die Probandinnen bekamen für die Bewertung keine Zeitvorgabe. Nach dem Experiment wurden die Probandinnen über die Ziele der Studie aufgeklärt. 


\subsubsection{Auswertung der Eye-Tracking-Ergebnisse}

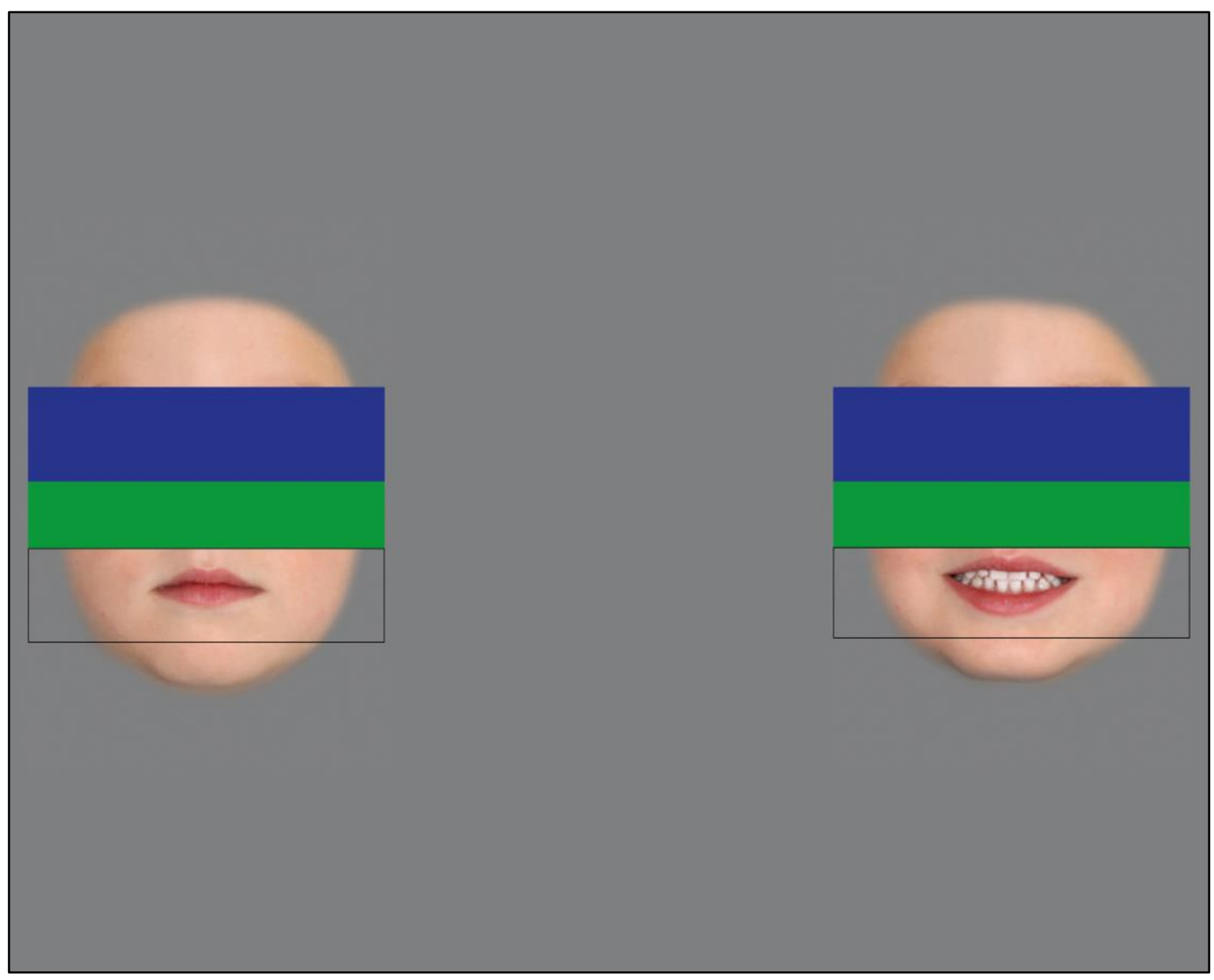

Abbildung 4.3: Beispiel eines Präsentationsbildes, links das neutrale Bild und rechts das emotionale Bild.

Augen und Nase sind aus datenschutzrechtlichen Gründen unkenntlich gemacht (modifiziert nach Tschammler et al. (2018), die Verwendung erfolgt mit freundlicher Genehmigung des Elsevier Verlags, Nachweis auf Seite 79).

Die Auswertung der Eye-Tracking-Untersuchungsdaten erfolgte mit Hilfe der

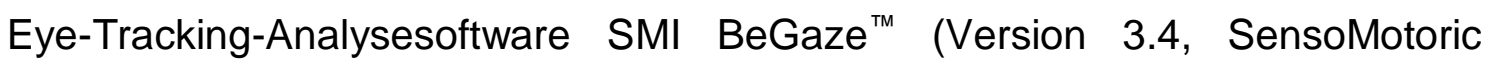
Instruments $\mathrm{GmbH}$, Teltow). Die zur Analyse definierten Einstellungen sind in Tabelle 4.2 zusammengefasst. Die Definition für die minimale Dauer eine Fixation variiert je nach Autor. Für die vorliegende Studie wurde sie auf $100 \mathrm{~ms}$ festgelegt und orientierte sich dabei an vergleichbaren Eye-TrackingExperimenten (van Schijndel et al. 2015; Dindaroğlu et al. 2017).

Die areas of interest (AOIs) Auge, Nase und Mund wurden über alle Fotos festgelegt. In Abbildung 4.3 ist dies exemplarisch für ein Präsentationsbildpaar dargestellt.

Die Auswertung der Gesamtfixationszeiten und der Fixationszählungen erfolgte unterteilt in die erste bis zweite Sekunde und für die dritte Sekunde, in der sich 
subjektiv interessantere Bereiche oder Regionen, die verstärkt Emotionen hervorrufen, durch eine stärkere Fokussierung bemerkbar machen können (Steinfurth et al. 2013).

Tabelle 4.2: Einstellungen der Eye-Tracking-Analysesoftware SMI BeGaze ${ }^{\mathrm{TM}}$.

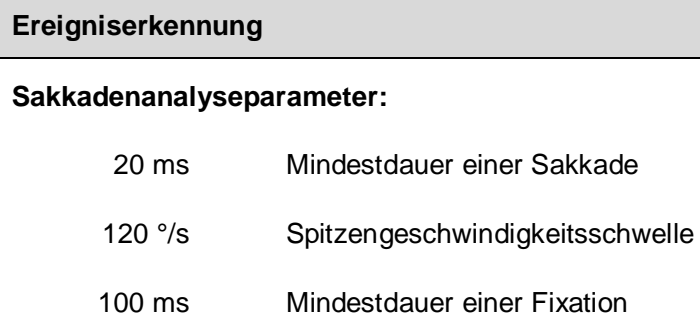

\subsection{Statistische Analyse}

Die Auswertung erfolgte mithilfe des Statistik-Programmes R (Version 3.4.0, The $\mathrm{R}$ Foundation for Statistical Computing; Wien, Österreich; www.r-project.org). Das Signifikanzniveau wurde auf $\alpha=0,05$ definiert.

Die Annahme, dass eine Normalverteilung der Daten vorlag, wurde nach Auswertung von Quantil-Quantil-Diagrammen und Kolmogorow-Smirnow-Tests abgelehnt, weshalb zur statistischen Analyse nichtparametrische Tests verwendet wurden. Die Verteilung der ersten Fixation wurde auf Unterschiede zwischen den Probandinnen-Gruppen und den Gebiss-Klassifikationen mit einem Binomialtest untersucht. Für Vergleiche der Gesamtfixationszeiten und Fixationsanzahlen zwischen den Probandinnen-Gruppen Experten und Laien wurden Mann-Whitney-U-Tests vorgenommen. Um Unterschiede zwischen den 
Gebiss-Klassifikationen zu analysieren, wurden Kruskal-Wallis-Tests mit Nemenyi Post-hoc-Tests gemacht. Unterschiede hinsichtlich Valenz, Arousal und Attraktivität wurden mithilfe von Mann-Whitney-U-Tests (für Unterschiede zwischen Experten und Laien) bzw. mit Kruskal-Wallis-Tests mit Nemenyi-Post-hoc-Tests (für Unterschiede zwischen den GebissKlassifikationen) analysiert. Bei multiplen Vergleichen erfolgte eine p-Wert-Korrektur mithilfe der Bonferroni-Methode. 


\section{Ergebnisse}

Insgesamt konnten 38 Probandinnen für die Teilnahme an dieser Eye-Tracking-Studie gewonnen werden. Die 18 zahnmedizinischen Laien wiesen im Durchschnitt ein Alter von 21,4 2,6 Jahren und die 20 Experten ein Alter von 23,9 $\pm 2,1$ Jahren auf. Der Händigkeitsquotient nach Oldfield (1971) der Laien lag bei $+77,0 \pm 18,0$ und der Experten bei $+76,0 \pm 16,7$. Somit setzten sich beide Gruppen aus rechtshändigen Probandinnen zusammen.

\subsection{Wahrnehmung von Kindergesichtern durch zahnmedizinische Laien und Experten}

\subsubsection{Ergebnisse der ersten Fixation}

Die erste Fixation als Marker für die initiale Aufmerksamkeit lag sowohl bei den zahnmedizinischen Laien $\left(p=0,001 ; p_{0}=0,534 ; p_{1}=0,466 ; C l 2,5 \%=0,439\right.$; $\mathrm{Cl} 97,5 \%=0,493)$ als auch bei den Experten ( $p<0,001 ; p_{0}=0,547$; $\left.\mathrm{p}_{1}=0,453 ; \mathrm{Cl} 2,5 \%=0,427 ; \mathrm{Cl} 97,5 \%=0,479\right)$ signifikant häufiger auf dem emotionalen Bild als auf dem neutralen Bild. Bei den zahnmedizinischen Laien fiel die erste Fixation am häufigsten auf die AOls Auge und Nase (emotionales und neutrales Bild) (Abbildung 5.1). Die erste Fixation der Experten fiel am häufigsten auf das AOI Mund des emotionalen Bildes. Im Vergleich der Probandinnen-Gruppen zeigte sich, dass sowohl auf dem neutralen als auch auf dem emotionalen Bild die Laien signifikant häufiger auf das AOI Auge schauten (neutral: $\quad p<0,001 ; \quad p_{0}=0,095 ; \quad p_{1}=0,196 ; \quad C l 2,5 \%=0,175$; Cl $97,5 \%=0,219 ; \quad$ emotional: $\quad p<0,001 ; \quad p_{0}=0,057 ; \quad p_{1}=0,193$; $\mathrm{Cl} 2,5 \%=0,173 ; \mathrm{Cl} 97,5 \%=0,215)$, während die Experten signifikant häufiger das $\mathrm{AOI}$ Mund des neutralen und emotionalen Bildes betrachteten (neutral: $\mathrm{p}<0,001 ; \quad \mathrm{p}_{0}=0,137 ; \quad \mathrm{p}_{1}=0,056 ; \quad \mathrm{Cl} 2,5 \%=0,044 ; \quad \mathrm{Cl} 97,5 \%=0,069$; emotional: $\quad p<0,001 ; \quad p_{0}=0,286 ; \quad p_{1}=0,118 ; \quad \mathrm{Cl} 2,5 \%=0,101$; $\mathrm{Cl} 97,5 \%=0,136)$. 


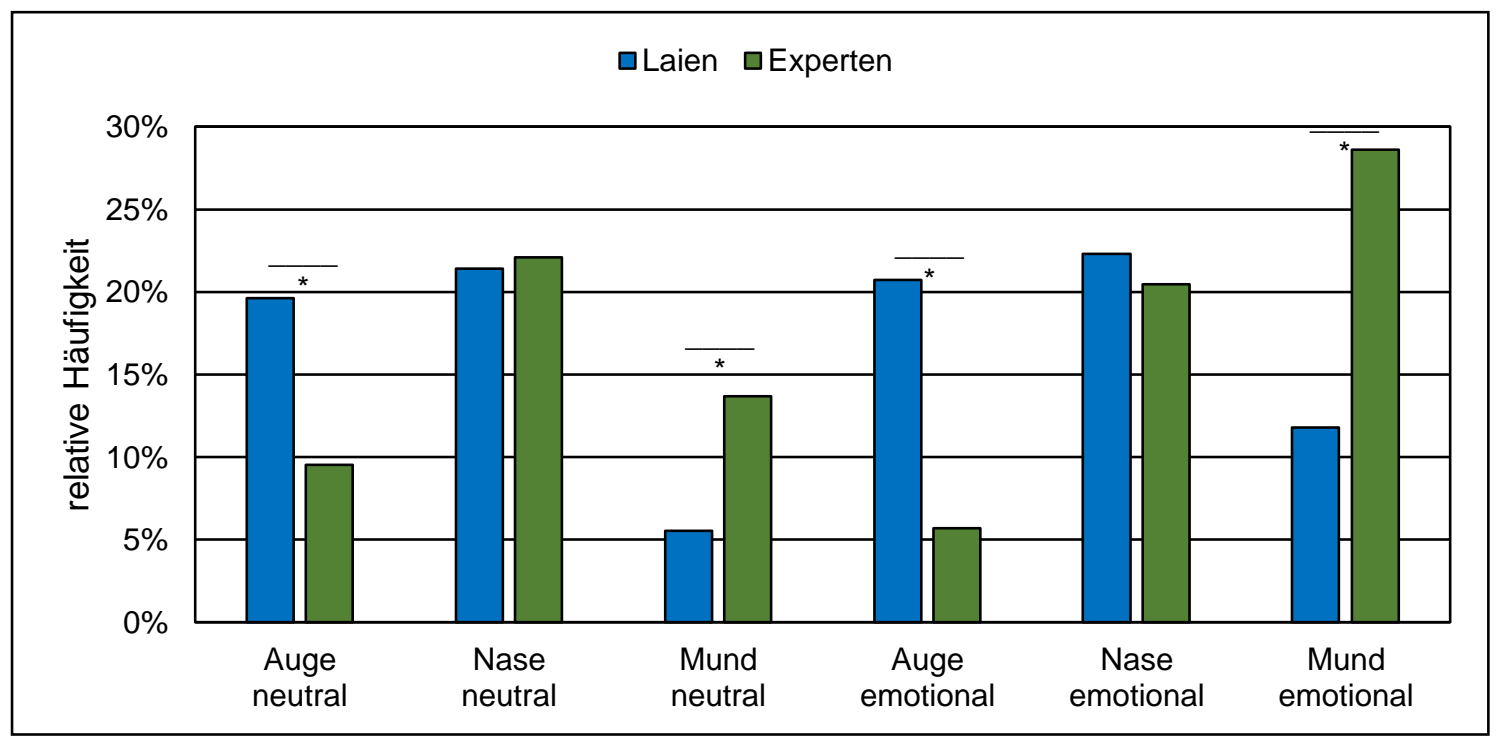

Abbildung 5.1: Relative Häufigkeit (\%) der 1. Fixation zwischen den Probandinnen-Gruppen. Die Probandinnen-Gruppen des gleichen Bildtyps und gleichen AOls wurden mit Binomialtests verglichen und signifikante Unterschiede zwischen den Probandinnen-Gruppen wurden mit Sternchen markiert.

\subsubsection{Ergebnisse der ersten bis zweiten Sekunde}

\subsubsection{Gesamtfixationszeiten}

In den ersten beiden Betrachtungssekunden fixierten beide ProbandinnenGruppen das emotionale Bild jeweils länger als das neutrale Bild (Abbildung 5.2). Im Vergleich zwischen zahnmedizinischen Experten und Laien zeigten die beiden Probandinnen-Gruppen auf den neutralen Bildern ähnliche Gesamtfixationszeiten für die AOls Nase und Mund. Das AOI Auge wurde jedoch von den zahnmedizinischen Laien signifikant länger betrachtet als von den Experten $(p=0,001 ; \quad W=231591,5 ; \quad z=66,0 ; \quad C l 2,5 \%=32,0 ;$ $\mathrm{Cl} 97,5 \%=101,0)$. Auf den emotionalen Bildern unterschied sich die Gesamtfixationsdauer der zahnmedizinischen Laien und Experten signifikant für alle AOls (Auge: $p<0,001 ; \quad W=266323,0 ; \quad z=92,0 ; \quad C l 2,5 \%=57,0$; Cl $97,5 \%=128,0 ; \quad$ Nase: $\quad p<0,001 ; \quad W=180598,0 ; \quad z=-140,0$; $\mathrm{Cl} 2,5 \%=-196,0 ; \quad \mathrm{Cl} 97,5 \%=-85,0 ; \quad$ Mund: $\quad p<0,001 ; \quad \mathrm{W}=147816,0$; $z=-388,0 ; \mathrm{Cl} 2,5 \%=-445,0 ; \mathrm{Cl} 97,5 \%=-388,0)$. Die Laien zeigten dabei eine ähnliche Verteilung der Gesamtfixationszeiten wie auf dem neutralen Bild (AOI Nase $>$ Auge $>$ Mund), wohingegen sich der Hauptfokus der Experten vom AOI Auge auf die Mundregion verschob. 

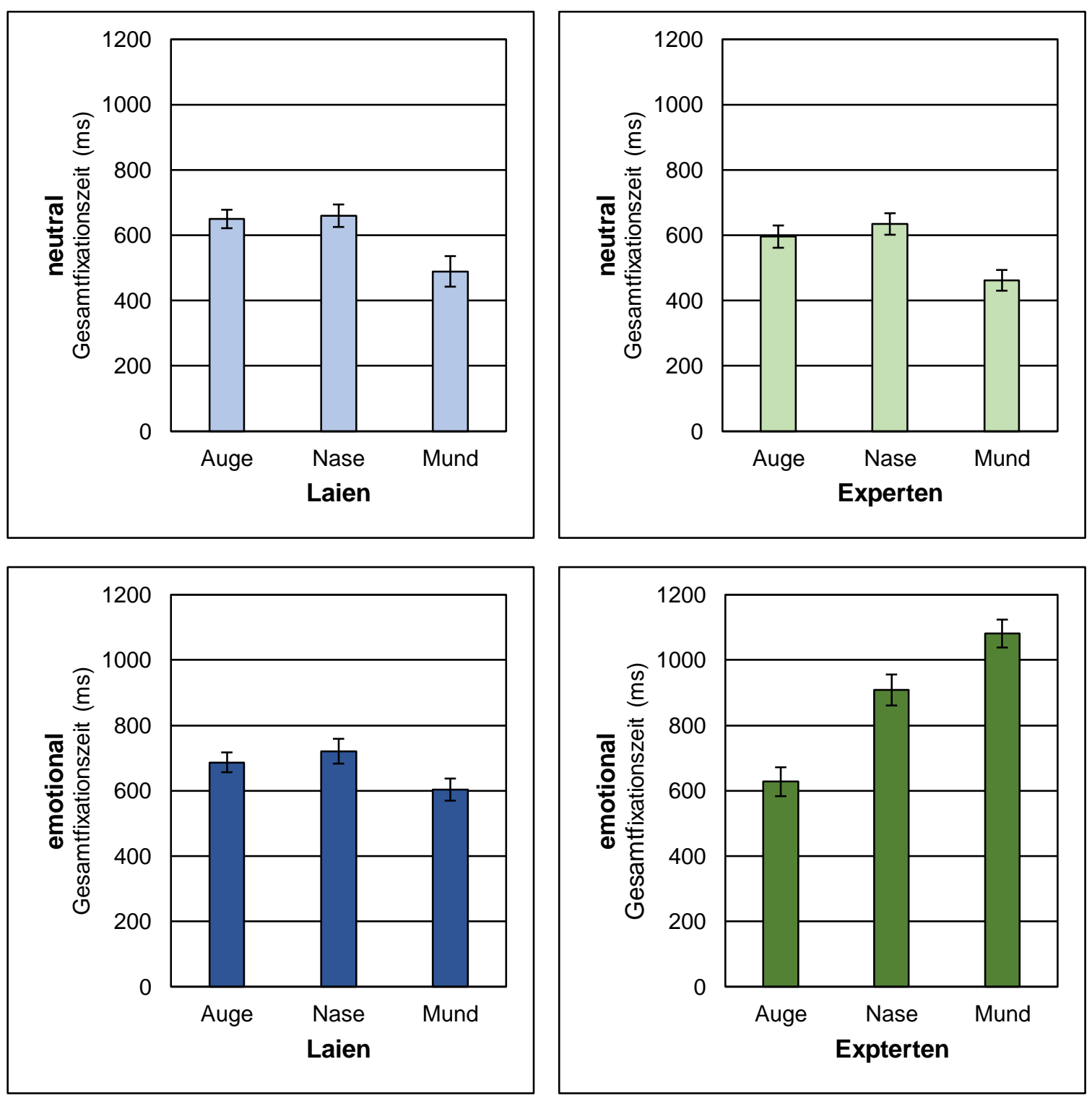

Abbildung 5.2: Mittelwerte der Gesamtfixationszeiten (ms) und 95\%-Konfidenzintervall für die 1. bis 2. Sekunde von Laien und Experten, dargestellt für die Bildtypen neutral und emotional.

\subsubsection{Anzahl der Fixationen}

Beide Probandinnen-Gruppen zeigten insgesamt eine ähnliche Anzahl an Fixationen während der ersten und zweiten Sekunde. Die zahnmedizinischen Laien fixierten dabei sowohl auf den neutralen als auch auf den emotionalen Bildern am häufigsten das AOI Auge (Tabelle 5.1). Auf dem neutralen Bild fielen die meisten Fixationen der Experten auf das AOI Nase und auf dem emotionalen Bild auf das AOI Mund. Die zahnmedizinischen Experten fixierten hingegen die Augenregion auf beiden Bildtypen signifikant kürzer (neutral: $\mathrm{p}<0,001 ; \quad \mathrm{W}=1287113,0 ; \quad \mathrm{z}=8,63^{*} 10^{-7} ; \quad \mathrm{Cl} 2,5 \%=6,75^{*} 10^{-5}$; CI $97,5 \%=1,82^{*} 10^{-5} ;$ emotional: $p<0,001 ; \quad W=1334017,0 ; \quad z=1,13^{*} 10^{-5}$; 
$\left.\mathrm{Cl} 2,5 \%=4,41^{*} 10^{-5} ; \mathrm{Cl} 97,5 \%=3,66^{*} 10^{-5}\right)$ und das AOI Mund dafür signifikant länger als die Laien (neutral: $p<0,001 ; W=966461,0 ; \quad z=-4,00^{*} 10^{-5}$; Cl $2,5 \%=-4,61 * 10^{-5} ; \quad$ Cl $97,5 \%=-1,41^{*} 10^{-5} ; \quad$ emotional: $\quad p<0,001$; $\left.W=865625,0 ; z=-2,26^{*} 10^{-5} ; \mathrm{Cl} 2,5 \%=-4,45^{\star} 10^{-5} ; \mathrm{Cl} 97,5 \%=-2,90^{*} 10^{-5}\right)$.

Tabelle 5.1: Vergleich der Anzahl der Fixationen in der 1. bis 2. Sekunde zwischen Laien und Experten. Die Anzahl der Fixationen im jeweiligen AOI wurde als Mittelwert (MW) mit dazugehörigem 95\%-Konfidenzintervall $(\mathrm{Cl})$ angegeben. Die Probandinnen-Gruppen wurden bei gleichem Bildtyp und gleichem AOI mit Mann-Whitney-U-Tests verglichen. Der $\mathrm{p}$-Wert wurde mit der Bonferroni-Methode korrigiert (modifiziert nach Tschammler et al. (2018), die Verwendung erfolgt mit freundlicher Genehmigung des Elsevier Verlags, Nachweis auf Seite 79).

\begin{tabular}{|c|c|c|c|c|}
\hline Bildtyp & $\mathrm{AOI}$ & $\begin{array}{c}\text { Laien } \\
\mathrm{MW}(\mathrm{Cl} 2,5 \% ; \mathrm{Cl} 97,5 \%)\end{array}$ & $\begin{array}{c}\text { Experten } \\
\mathrm{MW}(\mathrm{Cl} 2,5 \% ; \mathrm{Cl} 97,5 \%)\end{array}$ & Korr. p-Wert \\
\hline neutral & Auge & $0,70(0,66 ; 0,74)$ & $0,44(0,41 ; 0,47)$ & $<0,001$ \\
\hline neutral & Nase & $0,51(0,48 ; 0,55)$ & $0,51(0,48 ; 0,54)$ & 1,000 \\
\hline neutral & Mund & $0,21(0,19 ; 0,24)$ & $0,34(0,31 ; 0,37)$ & $<0,001$ \\
\hline emotional & Auge & $0,83(0,78 ; 0,88)$ & $0,48(0,44 ; 0,51)$ & $<0,001$ \\
\hline emotional & Nase & $0,54(0,50 ; 0,57)$ & $0,50(0,47 ; 0,54)$ & 1,000 \\
\hline emotional & Mund & $0,48(0,45 ; 0,51)$ & $0,74(0,70 ; 0,77)$ & $<0,001$ \\
\hline
\end{tabular}

\subsubsection{Ergebnisse der dritten Sekunde}

\subsubsection{Gesamtfixationszeiten}

Während der dritten Sekunde lag der größte Anteil der Gesamtfixationszeiten der zahnmedizinischen Laien auf dem AOI Nase beider Bildtypen. Die Gesamtfixationszeiten der dritten Sekunde fielen dabei kürzer aus als die Gesamtfixationszeiten der ersten und zweiten Sekunde (Abbildung 5.3). Beide Probandinnen-Gruppen zeigten dabei, sowohl auf den neutralen als auch auf den emotionalen Bildern, die gleiche Verteilung der Gesamtfixationszeiten auf die AOls wie in der ersten und zweiten Sekunde (Laien emotional und neutral, Experten neutral: AOI Nase > Auge > Mund; Experten emotional: AOI Mund > Nase > Auge). Im Vergleich zwischen Laien und Experten zeigten sich in der dritten Sekunde jedoch nur noch signifikante Unterschiede in Bezug auf das AOI Mund der emotionalen Bilder. Die Experten betrachteten dies signifikant länger als die Laien $(p<0,001 ; W=47866,0 ; z=-151,0 ; C l 2,5 \%=-195,0$; CI $97,5 \%=-106,0)$. 

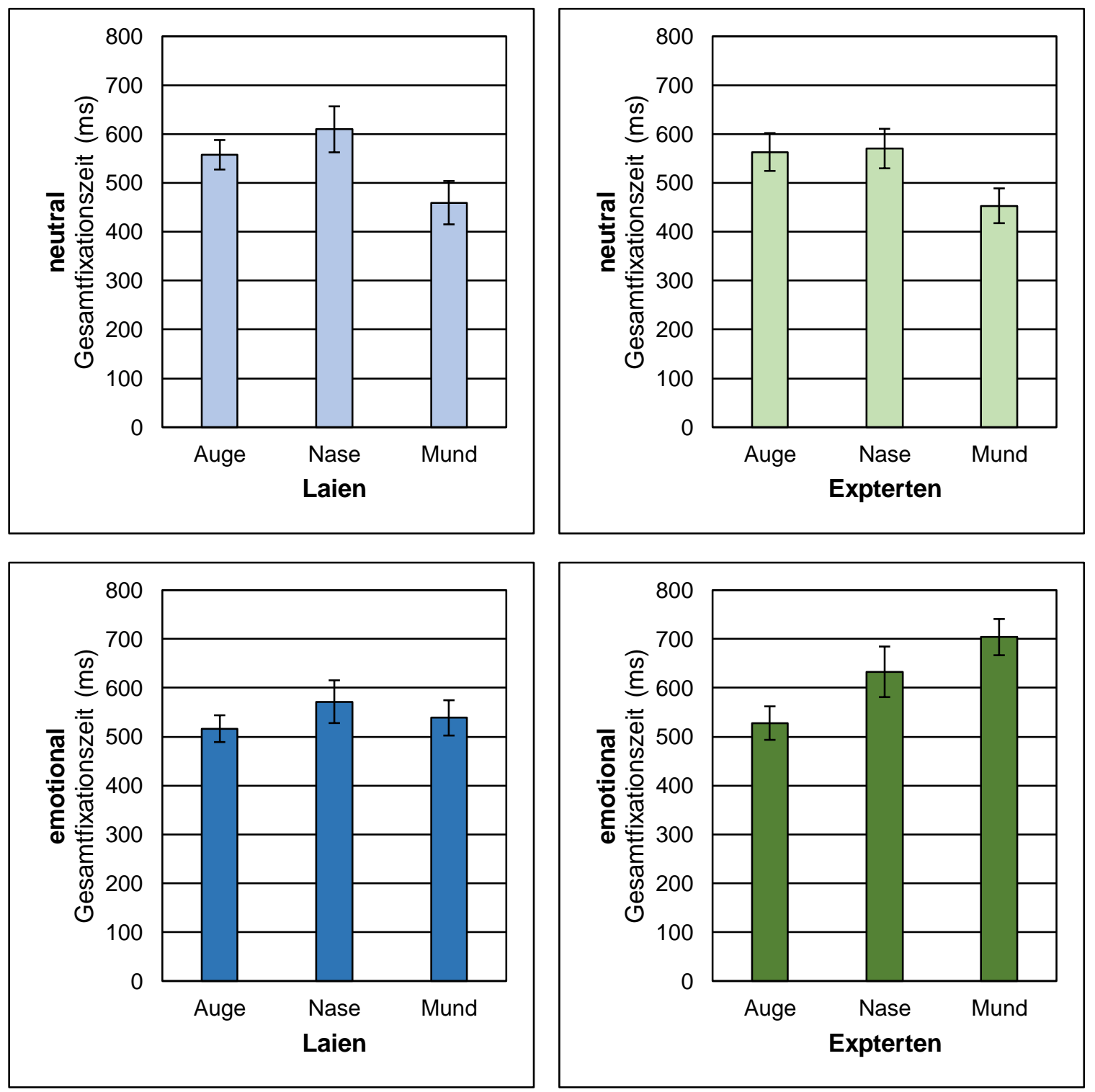

Abbildung 5.3: Mittelwerte der Gesamtfixationszeiten (ms) und 95\%-Konfidenzintervall für die 3. Sekunde von Laien und Experten, dargestellt für die Bildtypen neutral und emotional.

\subsubsection{Anzahl der Fixationen}

Wie in den ersten beiden Sekunden zeigten die Laien auch in der dritten Sekunde auf den neutralen und emotionalen Bildern die meisten Fixationen auf dem AOI Auge beider Bildtypen (neutral und emotional) (Tabelle 5.2).

Die zahnmedizinischen Experten schauten auf den emotionalen Bildern erneut überproportional häufig auf das $\mathrm{AOI}$ Mund. Auf den neutralen Bildern lag der Fokus in der dritten Sekunde im Gegensatz dazu auf dem AOI Auge und nicht mehr (wie in den ersten beiden Sekunden) auf dem AOI Nase.

Beide Probandinnen-Gruppen zeigten in Bezug auf die Anzahl der Fixationen bei beiden Bildertypen signifikante Unterschiede für das AOI Auge (neutral: 
$\mathrm{p}<0,001 ; \quad \mathrm{W}=1201835,5 ; \quad \mathrm{z}=6,01^{*} 10^{-5} ; \quad \mathrm{Cl} 2,5 \%=4,14^{*} 10^{-5}$;

CI 97,5\% $\%=2,44^{\star} 10^{-5} ;$ emotional: $p<0,001 ; \quad W=1180041,0 ; \quad z=7,85^{\star} 10^{-5}$; Cl $2,5 \%=9,19^{*} 10^{-5} ;$ Cl $97,5 \%=1,15^{*} 10^{-5}$ ) und auf dem emotionalen Bild ebenso für das AOI Mund $\left(p=0,012 ; \quad W=1041240,0 ; \quad z=-3,90^{*} 10^{-5}\right.$; $\left.\mathrm{Cl} 2,5 \%=-5,10^{*} 10^{-5} ; \mathrm{Cl} 97,5 \%=-5,71^{*} 10^{-6}\right)$. Diese Unterschiede zeigten sich schon bei der Analyse der Anzahl der Fixationen der ersten und zweiten Sekunde, jedoch gab es in der dritten Sekunde keinen signifikanten Unterschied zwischen beiden Probandinnen-Gruppen für das AOI Auge auf dem emotionalen Bild.

Tabelle 5.2: Vergleich der Anzahl der Fixationen in der 3. Sekunde zwischen Laien und Experten. Die Anzahl der Fixationen im jeweiligen $\mathrm{AOI}$ wurde als Mittelwert (MW) mit dazugehörigem 95\%-Konfidenzintervall (Cl) angegeben. Die Probandinnen-Gruppen wurden bei gleichem Bildtyp und gleichem AOI mit Mann-Whitney-U-Tests verglichen. Der $\mathrm{p}$-Wert wurde mit der Bonferroni-Methode korrigiert.

\begin{tabular}{|c|c|c|c|c|}
\hline Bildtyp & $\mathrm{AOI}$ & $\begin{array}{c}\text { Laien } \\
\mathrm{MW}(\mathrm{Cl} 2,5 \% ; \mathrm{Cl} 97,5 \%)\end{array}$ & $\begin{array}{c}\text { Experten } \\
\mathrm{MW}(\mathrm{Cl} 2,5 \% ; \mathrm{Cl} 97,5 \%)\end{array}$ & Korr. p-Wert \\
\hline neutral & Auge & $0,37(0,33 ; 0,40)$ & $0,23(0,21 ; 0,25)$ & $<0,001$ \\
\hline neutral & Nase & $0,20(0,18 ; 0,22)$ & $0,17(0,15 ; 0,19)$ & 0,701 \\
\hline neutral & Mund & $0,14(0,12 ; 0,16)$ & $0,16(0,14 ; 0,18)$ & 0,602 \\
\hline emotional & Auge & $0,35(0,32 ; 0,38)$ & $0,26(0,23 ; 0,28)$ & $<0,001$ \\
\hline emotional & Nase & $0,16(0,14 ; 0,19)$ & $0,16(0,14 ; 0,18)$ & 1,000 \\
\hline emotional & Mund & $0,24(0,21 ; 0,26)$ & $0,29(0,26 ; 0,31)$ & 0,012 \\
\hline
\end{tabular}

\subsection{Wahrnehmung von Kindergesichtern in Abhängigkeit vom dentalen Befund}

\subsubsection{Ergebnisse der ersten Fixation}

Im Vergleich der Gebissklassifikationen gegeneinander zeigte sich ein sehr homogenes Anschauverhalten der gleichen Bildtypen und AOls. Allerdings wurde von den Laien auf dem neutralen Bildtyp das AOI Mund der Kinder mit kariösen Zähnen signifikant häufiger angeschaut als das AOI Mund von Kindern nach Zahnsanierung $\left(p=0,025 ; p_{0}=0,038 ; p_{1}=0,071 ; \quad C l 2,5 \%=0,049\right.$; $\mathrm{Cl} 97,5 \%=0,099$; Abbildung 5.4). Ebenso wurde von den Laien auf dem neutralen Bild das AOI Nase bei Kindern mit gesunden Zähnen signifikant 
häufiger als erstes fixiert im Vergleich zu Kindern mit kariösen Gebissen $\left(p=0,045 ; p_{0}=0,187 ; p_{1}=0,248 ; C l 2,5 \%=0,209 ; C l 97,5 \%=0,291\right)$. Auf dem neutralen Bildtyp wurde das AOI Auge der Kinder mit gesunden Zähnen von den Experten signifikant häufiger zuerst betrachtet als das der Kinder mit kariösen Zähnen $\quad\left(p=0,008 ; \quad p_{0}=0,072 ; \quad p_{1}=0,119 ; \quad C l 2,5 \%=0,092\right.$; Cl 97,5\% = 0,151; Abbildung 5.5).

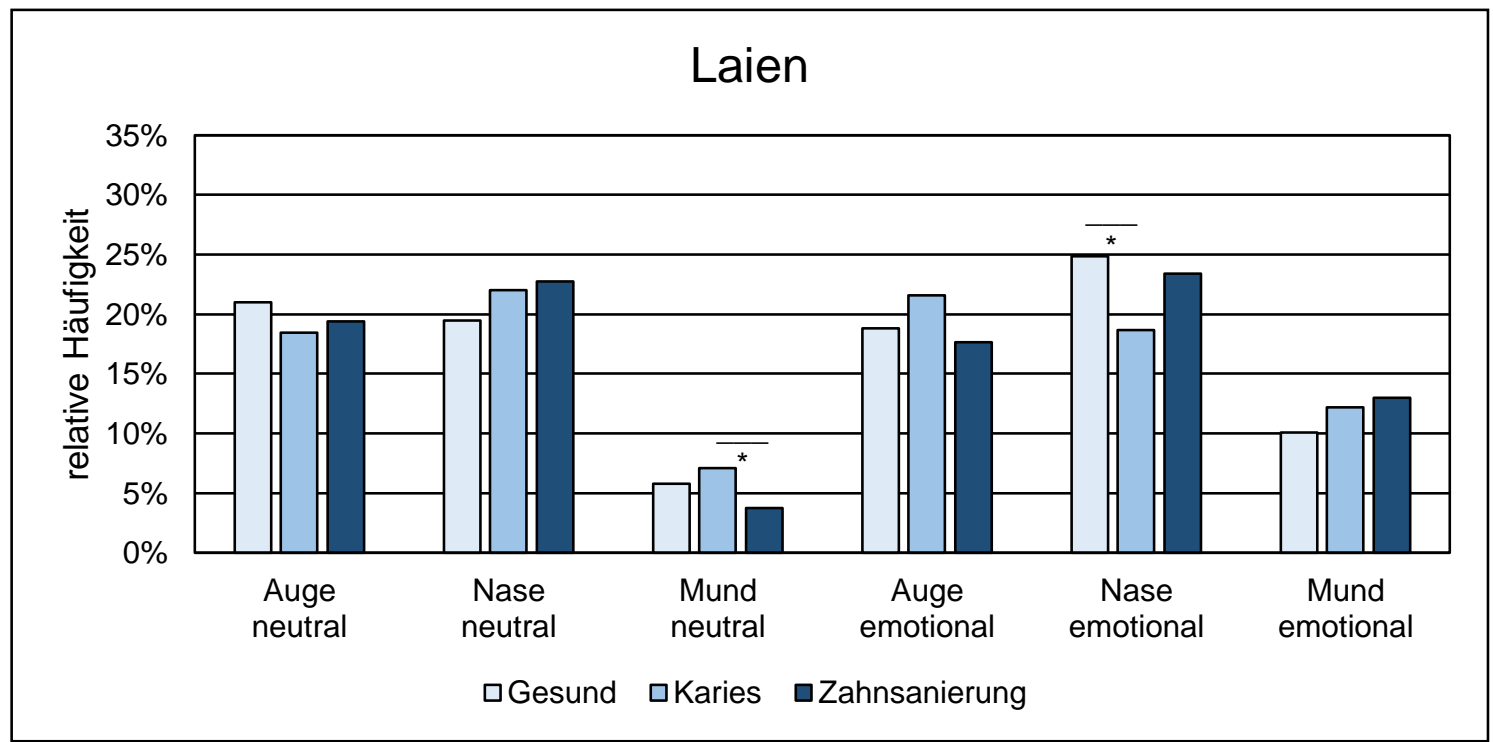

Abbildung 5.4: Relative Häufigkeit (\%) der 1. Fixation der Laien aufgeteilt entsprechend den Gebiss-Klassifikationen. Die Gebiss-Klassifizierungen wurden innerhalb des jeweiligen Bildtyps und des entsprechenden AOls mit Binomialtests verglichen und signifikante Unterschiede mit Sternchen gekennzeichnet.

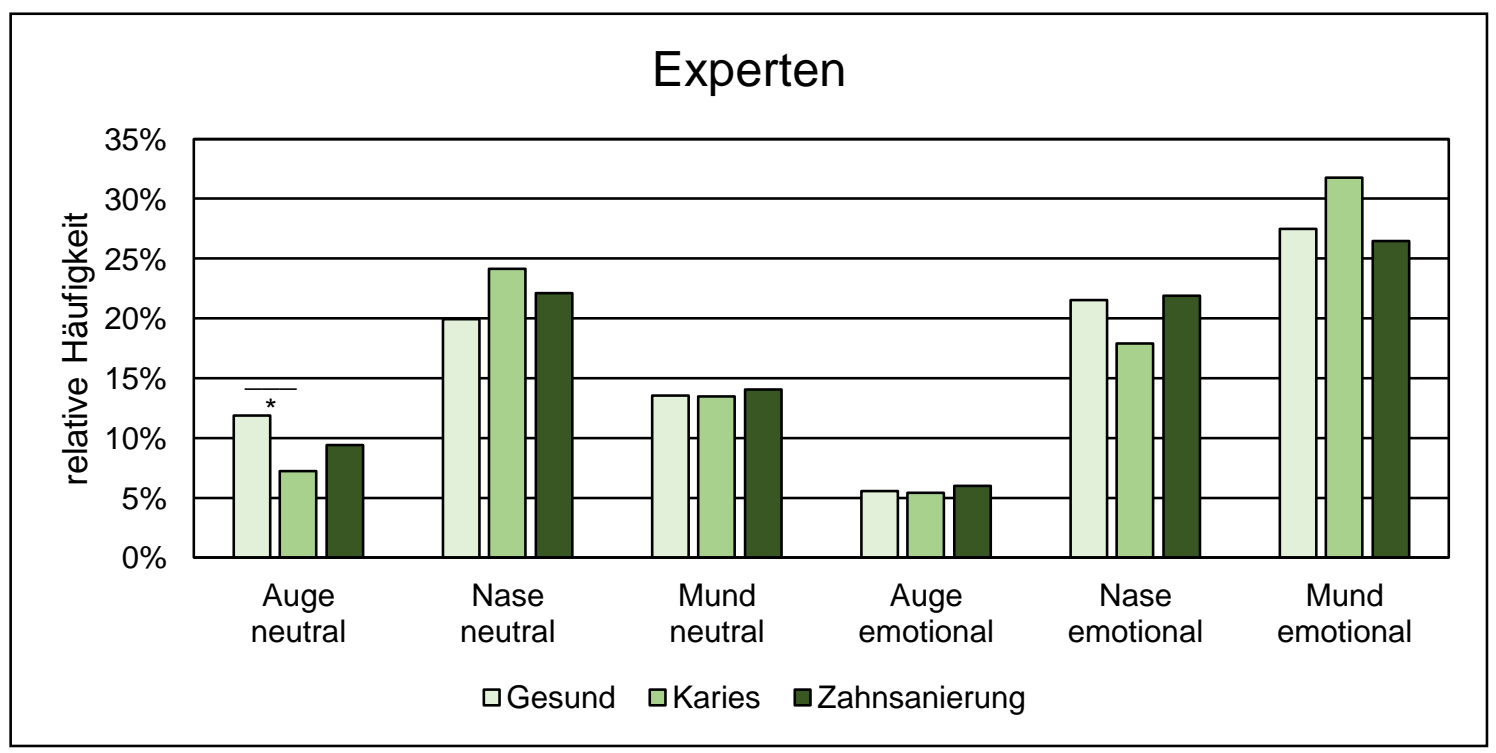

Abbildung 5.5: Relative Häufigkeit (\%) der 1. Fixation der Experten aufgeteilt entsprechend den Gebiss-Klassifikationen. Die Gebiss-Klassifizierungen wurden innerhalb des jeweiligen Bildtyps und des entsprechenden AOls mit Binomialtests verglichen und signifikante Unterschiede mit Sternchen gekennzeichnet. 


\subsubsection{Ergebnisse der ersten bis zweiten Sekunde}

In den ersten beiden Sekunden konnten bei den Laien keine signifikanten Unterschiede in Bezug auf die Gesamtfixationszeiten oder Fixationsanzahlen zwischen den Gebiss-Klassifizierungen „gesund“, „Karies“ und „Zahnsanierung“ festgestellt werden (Tabelle 5.3, Tabelle 5.4). Die zahnmedizinischen Experten fixierten auf den emotionalen Bildern das AOI Mund der Kinder mit kariösen Zähnen signifikant länger als auf den Bildern von Kindern mit gesunden Zähnen (Kruskal-Wallis-Test: $p=0,004 ; \chi^{2}=15,873$; df $=2$; Nemenyi-Post-hoc-Test: $p=0,008 ; \chi^{2}$-Verteilung $\left.=5,624\right)$. Es gab keine signifikanten Unterschiede im Vergleich der Anzahl der Fixationen zwischen den Gebiss-Klassifizierungen. 
Tabelle 5.3: Vergleich der Gesamtfixationszeiten in der 1. bis 2. Sekunde zwischen den Gebiss-Klassifizierungen „gesund“, „Karies“ und „Zahnsanierung“. Die Gesamtfixationszeiten (ms) wurden als Mittelwert (MW) mit dazugehörigem 95\%-Konfidenzintervall (Cl) angegeben. Die Gebiss-Klassifizierungen wurden innerhalb der gleichen Probandinnen-Gruppe, des gleichen Bildtyps und des gleichen AOls mit Kruskal-Wallis-Tests mit Nemenyi-Post-hoc-Tests verglichen. Der p-Wert wurde mit der Bonferroni-Methode korrigiert (modifiziert nach Tschammler et al. (2018), die Verwendung erfolgt mit freundlicher Genehmigung des Elsevier Verlags, Nachweis auf Seite 79).

\begin{tabular}{|c|c|c|c|c|c|c|c|c|c|}
\hline \multirow[t]{2}{*}{$\begin{array}{l}\text { Probandinnen- } \\
\text { Gruppe }\end{array}$} & \multirow[t]{2}{*}{ Bildtyp } & \multirow[t]{2}{*}{$\mathrm{AOI}$} & \multicolumn{3}{|c|}{ Gebiss-Klassifizierung } & \multirow[t]{2}{*}{$\begin{array}{l}\text { Korr. } \\
\text { p-Wert }\end{array}$} & \multicolumn{3}{|c|}{$\begin{array}{l}\text { Post-hoc Test } \\
\text { Korr. p-Wert }\end{array}$} \\
\hline & & & $\begin{array}{c}\text { Gesund } \\
\text { MW } \\
(\mathrm{Cl} 2,5 \% ; \mathrm{Cl} 97,5 \%)\end{array}$ & 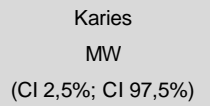 & $\begin{array}{c}\text { Zahnsanierung } \\
\text { MW } \\
\text { (Cl 2,5\%; Cl 97,5\%) }\end{array}$ & & $\begin{array}{l}\text { Gesund } \\
\text { vs. } \\
\text { Karies }\end{array}$ & $\begin{array}{c}\text { Gesund } \\
\text { vs. } \\
\text { Zahnsanierung }\end{array}$ & $\begin{array}{c}\text { Karies } \\
\text { vs. } \\
\text { Zahnsanierung }\end{array}$ \\
\hline Laien & neutral & Auge & $\begin{array}{c}643,29 \\
(596,66 ; 689,92)\end{array}$ & $\begin{array}{c}643,28 \\
(594,68 ; 691,89)\end{array}$ & $\begin{array}{c}664,15 \\
(611,76 ; 716,54)\end{array}$ & 1,000 & n. a. & n. a. & n. a. \\
\hline Laien & neutral & Nase & $\begin{array}{c}648,71 \\
(585,45 ; 711,98)\end{array}$ & $\begin{array}{c}656,71 \\
(599,35 ; 714,08)\end{array}$ & $\begin{array}{c}672,75 \\
(613,53 ; 731,98)\end{array}$ & 1,000 & n. a. & n. a. & n. a. \\
\hline Laien & neutral & Mund & $\begin{array}{c}451,56 \\
(372,32 ; 530,81)\end{array}$ & $\begin{array}{c}501,67 \\
(420,99 ; 582,35)\end{array}$ & $\begin{array}{c}515,15 \\
(429,48 ; 600,82)\end{array}$ & 1,000 & n. a. & n. a. & n. a. \\
\hline Laien & emotional & Auge & $\begin{array}{c}707,44 \\
(653,86 ; 761,01)\end{array}$ & $\begin{array}{c}642,90 \\
(592,35 ; 693,45)\end{array}$ & $\begin{array}{c}714,09 \\
(660,00 ; 768,18)\end{array}$ & 0,490 & n. a. & n. a. & n. a. \\
\hline Laien & emotional & Nase & $\begin{array}{c}723,55 \\
(660,26 ; 786,84)\end{array}$ & $\begin{array}{c}715,59 \\
(645,06 ; 786,11)\end{array}$ & $\begin{array}{c}723,03 \\
(658,39 ; 787,68)\end{array}$ & 1,000 & n. a. & n. a. & n. a. \\
\hline Laien & emotional & Mund & $\begin{array}{c}566,50 \\
(503,35 ; 629,64)\end{array}$ & $\begin{array}{c}610,61 \\
(560,26 ; 660,95)\end{array}$ & $\begin{array}{c}627,61 \\
(563,09 ; 692,13)\end{array}$ & 0,802 & n. a. & n. a. & n. a. \\
\hline Experten & neutral & Auge & $\begin{array}{c}620,19 \\
(560,88 ; 679,50)\end{array}$ & $\begin{array}{c}566,74 \\
(505,60 ; 627,89)\end{array}$ & $\begin{array}{c}595,84 \\
(538,55 ; 653,13)\end{array}$ & 1,000 & n. a. & n. a. & n. a. \\
\hline Experten & neutral & Nase & $\begin{array}{c}654,86 \\
(594,67 ; 715,04)\end{array}$ & $\begin{array}{c}590,24 \\
(537,72 ; 642,77)\end{array}$ & $\begin{array}{c}659,95 \\
(601,43 ; 718,46)\end{array}$ & 1,000 & n. a. & n. a. & n. a. \\
\hline Experten & neutral & Mund & $\begin{array}{c}440,85 \\
(391,37 ; 490,33)\end{array}$ & $\begin{array}{c}475,01 \\
(417,20 ; 532,82)\end{array}$ & $\begin{array}{c}471,55 \\
(413,12 ; 529,97)\end{array}$ & 1,000 & n. a. & n. a. & n. a. \\
\hline Experten & emotional & Auge & $\begin{array}{c}641,07 \\
(563,34 ; 718,79)\end{array}$ & $\begin{array}{c}610,78 \\
(534,51 ; 687,05)\end{array}$ & $\begin{array}{c}629,59 \\
(552,51 ; 706,68)\end{array}$ & 1,000 & n. a. & n. a. & n. a. \\
\hline Experten & emotional & Nase & $\begin{array}{c}899,45 \\
(816,85 ; 982,04)\end{array}$ & $\begin{array}{c}894,06 \\
(810,48 ; 977,63)\end{array}$ & $\begin{array}{c}931,76 \\
(850,39 ; 1013,13)\end{array}$ & 1,000 & n. a. & n. a. & n. a. \\
\hline Experten & emotional & Mund & $\begin{array}{c}968,05 \\
(899,63 ; 1036,48)\end{array}$ & $\begin{array}{c}1187,55 \\
(1109,44 ; 1265,67)\end{array}$ & $\begin{array}{c}1082,40 \\
(1009,07 ; 1155,73)\end{array}$ & 0,004 & 0,008 & 1,000 & 1,000 \\
\hline
\end{tabular}


Tabelle 5.4: Vergleich der Anzahl der Fixationen in der 1. bis 2. Sekunde zwischen den Gebiss-Klassifizierungen „gesund“, „Karies“ und „Zahnsanierung“. Die Anzahl der Fixationen wurden als Mittelwert (MW) mit dazugehörigem 95\%-Konfidenzintervall $(\mathrm{Cl})$ angegeben. Die Gebiss-Klassifizierungen wurden bei der gleichen Probandinnen-Gruppe, des gleichen Bildtyps und des gleichen AOls mit KruskalWallis-Tests mit Nemenyi-Post-hoc-Tests verglichen. Der p-Wert wurde mit der Bonferroni-Methode korrigiert (modifiziert nach Tschammler et al. (2018), die Verwendung erfolgt mit freundlicher Genehmigung des Elsevier Verlags, Nachweis auf Seite 79).

\begin{tabular}{|c|c|c|c|c|c|c|c|c|c|}
\hline \multirow[t]{2}{*}{$\begin{array}{l}\text { Probandinnen- } \\
\text { Gruppe }\end{array}$} & \multirow[t]{2}{*}{ Bildtyp } & \multirow[t]{2}{*}{$\mathrm{AOI}$} & \multicolumn{3}{|c|}{ Gebiss-Klassifizierung } & \multirow[t]{2}{*}{$\begin{array}{l}\text { Korr. } \\
\text { p-Wert }\end{array}$} & \multicolumn{3}{|c|}{$\begin{array}{l}\text { Post-hoc Test } \\
\text { Korr. p-Wert }\end{array}$} \\
\hline & & & $\begin{array}{c}\text { Gesund } \\
\text { MW } \\
(\mathrm{Cl} 2,5 \% ; \mathrm{Cl} 97,5 \%)\end{array}$ & $\begin{array}{c}\text { Karies } \\
\text { MW } \\
(\mathrm{Cl} 2,5 \% ; \mathrm{Cl} 97,5 \%)\end{array}$ & $\begin{array}{c}\text { Zahnsanierung } \\
\text { MW } \\
(\mathrm{Cl} 2,5 \% ; \mathrm{Cl} 97,5 \%)\end{array}$ & & $\begin{array}{l}\text { Gesund } \\
\text { vs. } \\
\text { Karies }\end{array}$ & $\begin{array}{c}\text { Gesund } \\
\text { vs. } \\
\text { Zahnsanierung }\end{array}$ & $\begin{array}{c}\text { Karies } \\
\text { vs. } \\
\text { Zahnsanierung }\end{array}$ \\
\hline Laien & neutral & Auge & $0,75(0,68 ; 0,82)$ & $0,67(0,60 ; 0,74)$ & $0,68(0,61 ; 0,75)$ & 1,000 & n. a. & n. a. & n. a. \\
\hline Laien & neutral & Nase & $0,49(0,43 ; 0,55)$ & $0,50(0,44 ; 0,56)$ & $0,54(0,49 ; 0,60)$ & 1,000 & n. a. & n. a. & n. a. \\
\hline Laien & neutral & Mund & $0,20(0,16 ; 0,24)$ & $0,25(0,20 ; 0,29)$ & $0,19(0,15 ; 0,23)$ & 1,000 & n. a. & n. a. & n. a. \\
\hline Laien & emotional & Auge & $0,84(0,75 ; 0,92)$ & $0,82(0,75 ; 0,90)$ & $0,83(0,74 ; 0,91)$ & 1,000 & n. a. & n. a. & n. a. \\
\hline Laien & emotional & Nase & $0,59(0,53 ; 0,66)$ & $0,49(0,43 ; 0,54)$ & $0,54(0,48 ; 0,60)$ & 0,798 & n. a. & n. a. & n. a. \\
\hline Laien & emotional & Mund & $0,40(0,35 ; 0,46)$ & $0,56(0,49 ; 0,62)$ & $0,48(0,42 ; 0,54)$ & 0,018 & 0,180 & 1,000 & 1,000 \\
\hline Experten & neutral & Auge & $0,50(0,44 ; 0,56)$ & $0,39(0,34 ; 0,44)$ & $0,44(0,38 ; 0,49)$ & 0,427 & n. a. & n. a. & n. a. \\
\hline Experten & neutral & Nase & $0,52(0,47 ; 0,58)$ & $0,51(0,45 ; 0,56)$ & $0,49(0,44 ; 0,55)$ & 1,000 & n. a. & n. a. & n. a. \\
\hline Experten & neutral & Mund & $0,35(0,30 ; 0,40)$ & $0,34(0,30 ; 0,39)$ & $0,33(0,28 ; 0,38)$ & 1,000 & n. a. & n. a. & n. a. \\
\hline Experten & emotional & Auge & $0,49(0,43 ; 0,55)$ & $0,45(0,39 ; 0,51)$ & $0,49(0,43 ; 0,56)$ & 1,000 & n. a. & n. a. & n. a. \\
\hline Experten & emotional & Nase & $0,53(0,47 ; 0,58)$ & $0,50(0,45 ; 0,55)$ & $0,48(0,43 ; 0,54)$ & 1,000 & n. a. & n. a. & n. a. \\
\hline Experten & emotional & Mund & $0,72(0,66 ; 0,78)$ & $0,75(0,70 ; 0,81)$ & $0,74(0,68 ; 0,80)$ & 1,000 & n. a. & n. a. & n. a. \\
\hline
\end{tabular}




\subsubsection{Ergebnisse der dritten Sekunde}

Während der dritten Sekunde ergaben sich in Bezug auf die Gebiss-Klassifizierungen weder bei den Laien noch bei den Experten signifikante Unterschiede in den Gesamtfixationszeiten oder den Fixationsanzahlen für den jeweiligen Bildtyp und das jeweilige AOI (Tabelle 5.5, Tabelle 5.6).

Tabelle 5.5: Vergleich der Gesamtfixationszeiten in der 3. Sekunde zwischen den Gebiss-Klassifizierungen „gesund“, „Karies“ und „Zahnsanierung“. Die Gesamtfixationszeiten (ms) wurden als Mittelwert (MW) mit dazugehörigem 95\%-Konfidenzintervall (Cl) angegeben. Die Gebiss-Klassifizierungen bei gleicher Probandinnen-Gruppe, gleichem Bildtyp und gleichem AOI wurden mit Kruskal-Wallis-Tests mit Nemenyi-Post-hoc-Tests verglichen. Der p-Wert wurde mit der BonferroniMethode korrigiert.

\begin{tabular}{|c|c|c|c|c|c|c|c|c|c|}
\hline \multirow{2}{*}{$\begin{array}{l}\text { Probandinnen- } \\
\text { Gruppe }\end{array}$} & \multirow[t]{2}{*}{ Bildtyp } & \multirow[t]{2}{*}{$\mathrm{AOI}$} & \multicolumn{3}{|c|}{ Gebiss-Klassifizierung } & \multirow{2}{*}{$\begin{array}{l}\text { Korr. } \\
\text { p-Wert }\end{array}$} & \multicolumn{3}{|c|}{$\begin{array}{l}\text { Post-hoc Test } \\
\text { Korr. p-Wert }\end{array}$} \\
\hline & & & $\begin{array}{c}\text { Gesund } \\
\text { MW } \\
(\mathrm{Cl} 2,5 \% ; \mathrm{Cl} 97,5 \%)\end{array}$ & 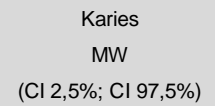 & $\begin{array}{c}\text { Zahnsanierung } \\
\text { MW } \\
(\mathrm{Cl} 2,5 \% ; \mathrm{Cl} 97,5 \%)\end{array}$ & & $\begin{array}{c}\text { Gesund } \\
\text { vs. } \\
\text { Karies }\end{array}$ & $\begin{array}{c}\text { Gesund } \\
\text { vs. } \\
\text { Zahnsanierung }\end{array}$ & $\begin{array}{c}\text { Karies } \\
\text { vs. } \\
\text { Zahnsanierung }\end{array}$ \\
\hline Laien & neutral & Auge & $\begin{array}{c}544,76 \\
(494,49 ; 595,03)\end{array}$ & $\begin{array}{c}580,2 \\
(522,54 ; 637,86)\end{array}$ & $\begin{array}{c}549,81 \\
(499,93 ; 599,70)\end{array}$ & 1,000 & n. a. & n. a. & n. a. \\
\hline Laien & neutral & Nase & $\begin{array}{c}595,69 \\
(506,19 ; 685,20)\end{array}$ & $\begin{array}{c}655,02 \\
(564,20 ; 745,84)\end{array}$ & $\begin{array}{c}575,64 \\
(510,69 ; 640,60)\end{array}$ & 1,000 & n. a. & n. a. & n. a. \\
\hline Laien & neutral & Mund & $\begin{array}{c}483,42 \\
(424,01 ; 542,82)\end{array}$ & $\begin{array}{c}401,06 \\
(344,60 ; 457,52)\end{array}$ & $\begin{array}{c}497,20 \\
(379,16 ; 615,24)\end{array}$ & 1,000 & n. a. & n. a. & n. a. \\
\hline Laien & emotional & Auge & $\begin{array}{c}528,76 \\
(472,04 ; 585,48)\end{array}$ & $\begin{array}{c}526,71 \\
(479,80 ; 573,62)\end{array}$ & $\begin{array}{c}495,63 \\
(456,75 ; 534,51)\end{array}$ & 1,000 & n. a. & n. a. & n. a. \\
\hline Laien & emotional & Nase & $\begin{array}{c}581,51 \\
(497,22 ; 665,81)\end{array}$ & $\begin{array}{c}568,11 \\
(492,14 ; 644,08)\end{array}$ & $\begin{array}{c}564,03 \\
(496,95 ; 631,10)\end{array}$ & 1,000 & n. a. & n. a. & n. a. \\
\hline Laien & emotional & Mund & $\begin{array}{c}551,62 \\
(485,56 ; 617,68)\end{array}$ & $\begin{array}{c}536,76 \\
(478,24 ; 595,27)\end{array}$ & $\begin{array}{c}525,67 \\
(460,35 ; 591,00)\end{array}$ & 1,000 & n. a. & n. a. & n. a. \\
\hline Experten & neutral & Auge & $\begin{array}{c}560,17 \\
(490,26 ; 630,08)\end{array}$ & $\begin{array}{c}562,05 \\
(498,83 ; 625,28)\end{array}$ & $\begin{array}{c}567,69 \\
(496,86 ; 638,51)\end{array}$ & 1,000 & n. a. & n. a. & n. a. \\
\hline Experten & neutral & Nase & $\begin{array}{c}585,86 \\
(521,27 ; 650,45)\end{array}$ & $\begin{array}{c}578,46 \\
(503,79 ; 653,14)\end{array}$ & $\begin{array}{c}542,23 \\
(467,70 ; 616,77)\end{array}$ & 1,000 & n. a. & n. a. & n. a. \\
\hline Experten & neutral & Mund & $\begin{array}{c}410,16 \\
(351,29 ; 469,04)\end{array}$ & $\begin{array}{c}453,51 \\
(391,60 ; 515,42)\end{array}$ & $\begin{array}{c}494,30 \\
(429,17 ; 559,44)\end{array}$ & 1,000 & n. a. & n. a. & n. a. \\
\hline Experten & emotional & Auge & $\begin{array}{c}543,51 \\
(481,98 ; 605,03)\end{array}$ & $\begin{array}{c}518,32 \\
(454,19 ; 582,44)\end{array}$ & $\begin{array}{c}521,65 \\
(467,55 ; 575,74)\end{array}$ & 1,000 & n. a. & n. a. & n. a. \\
\hline Experten & emotional & Nase & $\begin{array}{c}644,86 \\
(542,41 ; 747,32)\end{array}$ & $\begin{array}{c}642,92 \\
(545,47 ; 740,37)\end{array}$ & $\begin{array}{c}611,21 \\
(541,33 ; 681,09)\end{array}$ & 1,000 & n. a. & n. a. & n. a. \\
\hline Experten & emotional & Mund & $\begin{array}{c}672,73 \\
(612,10 ; 733,37)\end{array}$ & $\begin{array}{c}731,95 \\
(661,97 ; 801,92)\end{array}$ & $\begin{array}{c}711,84 \\
(648,09 ; 775,60)\end{array}$ & 1,000 & n. a. & n. a. & n. a. \\
\hline
\end{tabular}


Tabelle 5.6: Vergleich der Anzahl der Fixationen in der 3. Sekunde zwischen den Gebiss-Klassifizierungen „gesund“, „Karies“ und „Zahnsanierung“. Die Anzahl der Fixationen wurde als Mittelwert (MW) mit dazugehörigem 95\%-Konfidenzintervall (Cl) angegeben. Die Gebiss-Klassifizierungen bei gleicher Probandinnen-Gruppe, gleichem Bildtyp und gleichem AOI wurden mit Kruskal-Wallis-Tests mit NemenyiPost-hoc-Tests verglichen. Der $p$-Wert wurde mit der Bonferroni-Methode korrigiert.

\begin{tabular}{|c|c|c|c|c|c|c|c|c|c|}
\hline \multirow{2}{*}{$\begin{array}{c}\text { Probandinnen- } \\
\text { Gruppe }\end{array}$} & \multirow[t]{2}{*}{ Bildtyp } & \multirow[t]{2}{*}{$\mathrm{AOI}$} & \multicolumn{3}{|c|}{ Gebiss-Klassifizierung } & \multirow{2}{*}{$\begin{array}{l}\text { Korr. } \\
p \text {-Wert }\end{array}$} & \multicolumn{3}{|c|}{$\begin{array}{l}\text { Post-hoc Test } \\
\text { Korr. p-Wert }\end{array}$} \\
\hline & & & $\begin{array}{c}\text { Gesund } \\
\text { MW } \\
(\mathrm{Cl} 2,5 \% ; \mathrm{Cl} 97,5 \%)\end{array}$ & $\begin{array}{c}\text { Karies } \\
\text { MW } \\
(\mathrm{Cl} 2,5 \% ; \mathrm{Cl} 97,5 \%)\end{array}$ & $\begin{array}{c}\text { Zahnsanierung } \\
\text { MW } \\
(\mathrm{Cl} 2,5 \% ; \mathrm{Cl} 97,5 \%)\end{array}$ & & $\begin{array}{c}\text { Gesund } \\
\text { vs. } \\
\text { Karies }\end{array}$ & $\begin{array}{c}\text { Gesund } \\
\text { vs. } \\
\text { Zahnsanierung }\end{array}$ & $\begin{array}{c}\text { Karies } \\
\text { vs. } \\
\text { Zahnsanierung }\end{array}$ \\
\hline Laien & neutral & Auge & $0,41(0,35 ; 0,46)$ & $0,33(0,28 ; 0,38)$ & $0,36(0,30 ; 0,42)$ & 1,000 & n. a. & n. a. & n. a. \\
\hline Laien & neutral & Nase & $0,18(0,14 ; 0,22)$ & $0,21(0,17 ; 0,25)$ & $0,21(0,17 ; 0,25)$ & 1,000 & n. a. & n. a. & n. a. \\
\hline Laien & neutral & Mund & $0,16(0,13 ; 0,20)$ & $0,14(0,11 ; 0,18)$ & $0,12(0,09 ; 0,15)$ & 1,000 & n. a. & n. a. & n. a. \\
\hline Laien & emotional & Auge & $0,37(0,31 ; 0,42)$ & $0,32(0,27 ; 0,37)$ & $0,36(0,31 ; 0,41)$ & 1,000 & n. a. & n. a. & n. a. \\
\hline Laien & emotional & Nase & $0,19(0,15 ; 0,23)$ & $0,14(0,11 ; 0,18)$ & $0,16(0,13 ; 0,20)$ & 1,000 & n. a. & n. a. & n. a. \\
\hline Laien & emotional & Mund & $0,24(0,20 ; 0,29)$ & $0,26(0,21 ; 0,30)$ & $0,21(0,17 ; 0,25)$ & 1,000 & n. a. & n. a. & n. a. \\
\hline Experten & neutral & Auge & $0,22(0,18 ; 0,26)$ & $0,24(0,20 ; 0,28)$ & $0,23(0,19 ; 0,27)$ & 1,000 & n. a. & n. a. & n. a. \\
\hline Experten & neutral & Nase & $0,20(0,16 ; 0,23)$ & $0,17(0,13 ; 0,20)$ & $0,16(0,12 ; 0,19)$ & 1,000 & n. a. & n. a. & n. a. \\
\hline Experten & neutral & Mund & $0,15(0,12 ; 0,19)$ & $0,17(0,14 ; 0,20)$ & $0,17(0,13 ; 0,20)$ & 1,000 & n. a. & n. a. & n. a. \\
\hline Experten & emotional & Auge & $0,27(0,22 ; 0,32)$ & $0,23(0,19 ; 0,27)$ & $0,26(0,21 ; 0,31)$ & 1,000 & n. a. & n. a. & n. a. \\
\hline Experten & emotional & Nase & $0,16(0,13 ; 0,19)$ & $0,16(0,12 ; 0,19)$ & $0,16(0,13 ; 0,19)$ & 1,000 & n. a. & n. a. & n. a. \\
\hline Experten & emotional & Mund & $0,31(0,27 ; 0,36)$ & $0,26(0,22 ; 0,30)$ & $0,29(0,25 ; 0,33)$ & 1,000 & n. a. & n. a. & n. a. \\
\hline
\end{tabular}

\subsection{Ergebnisse der Fragebögen}

Im Anschluss an die Eye-Tracking-Untersuchung wurden die Fotos der Patienten von den Probandinnen mittels Fragebögen nach Valenz, Arousal und Attraktivität beurteilt.

\subsubsection{Vergleich der Probandinnen-Gruppen}

Die Laien bewerteten die gezeigten neutralen Fotos sehr ähnlich wie die Experten. Die Auswertung der Ergebnisse in Bezug auf Valenz und Arousal ergab für die einzelnen Gebiss-Klassifizierungen keine signifikanten Unterschiede zwischen den Probandinnen-Gruppen. In Bezug auf die Attraktivität der abgebildeten Kinder unterschieden sich die beiden Probandinnen-Gruppen hingegen signifikant. So bewerteten die Experten die Kinder mit gesunden Zähnen signifikant attraktiver als die Laien ( $p=0,041$; $W=25650,0 ; z=-0,99999 ; \mathrm{Cl} 2,5 \%=-0,99995 ;$ Cl 97,5\% $\%=-0,00005 ;$ Tabelle 5.8). 
Hinsichtlich der emotionalen Bilder zeigten sich deutlich mehr Unterschiede zwischen den Probandinnen-Gruppen. In der Bewertung der Bilder nach Valenz unterschieden sich die Probandinnen-Gruppen für alle drei Gebiss-Klassifizierungen signifikant voneinander. Die Experten bewerteten alle drei Gruppen signifikant negativer als die Laien (gesund: $p<0,001$; $W=23489,0 ; z=-0,99999 ; \mathrm{Cl} 2,5 \%=-1,0003 ; \mathrm{Cl} 97,5 \%=-0,00006$; Karies: $\mathrm{p}<0,001 ; \quad \mathrm{W}=14057,5 ; \quad \mathrm{z}=-2,00000 ; \quad \mathrm{Cl} 2,5 \%=-2,99993$; $\mathrm{Cl} 97,5 \%=-1,99995 ;$ Zahnsanierung: $p<0,001 ; \mathrm{W}=19854,0 ; z=-1,00004$; $\mathrm{Cl} 2,5 \%=-1,9995 ; \mathrm{Cl} 97,5 \%=-0,99992)$. Die Kinder mit kariösen Zähnen $(p<0,001 ; \quad W=22647,5 ; \quad z=-0,99996 ; \quad C l 2,5 \%=-1,00003$; $\mathrm{Cl} 97,5 \%=-1,00000)$ und die Kinder mit Zahnsanierung ( $p=0,034$; $W=25546,5 ; \mathrm{z}=-0,99997 ; \mathrm{Cl} 2,5 \%=-1,00000 ; \mathrm{Cl} 97,5 \%=-0,00006)$ wurden durch die Laien signifikant weniger erregend beurteilt als durch die Experten. Die Attraktivität der Kinder mit kariösen Zähnen wurde von den Experten als signifikant schlechter bewertet im Vergleich zu den Laien $(p<0,001$; $W=39773,0 ; z=1,00002 ; \mathrm{Cl} 2,5 \%=0,99998 ; \mathrm{Cl} 97,5 \%=1,00007)$. 
Tabelle 5.7: Valenz, Arousal und Attraktivität der Kinder mit gesunden Zähnen, mit kariösen Zähnen und mit Zahnsanierung beurteilt durch Laien und Experten auf neutralen und emotionalen Bildern. Die Antworten wurden als Mittelwert (MW) mit dazugehörigem 95\%-Konfidenzintervall (Cl) angegeben. Die Probandinnen-Gruppen bei gleicher Dimension, gleichem Bildtyp und gleicher Gebiss-Klassifizierung wurden mit Mann-Whitney-U-Tests verglichen. Der p-Wert wurde mit der Bonferroni-Methode korrigiert (modifiziert nach Tschammler et al. (2018), die Verwendung erfolgt mit freundlicher Genehmigung des Elsevier Verlags, Nachweis auf Seite 79).

\begin{tabular}{|c|c|c|c|c|c|}
\hline Emotion & Frage & Klassifizierung & $\begin{array}{c}\text { Laien } \\
\text { MW } \\
(\mathrm{Cl} 2,5 \% ; \mathrm{Cl} 97,5 \%)\end{array}$ & $\begin{array}{c}\text { Experten } \\
\text { MW } \\
(\mathrm{Cl} 2,5 \% ; \mathrm{Cl} 97,5 \%)\end{array}$ & Korr. p-Wert \\
\hline neutral & Valenz & Gesund & $4,16(3,96 ; 4,37)$ & $4,49(4,34 ; 4,64)$ & 0,176 \\
\hline neutral & Valenz & Karies & $4,39(4,11 ; 4,68)$ & $4,62(4,42 ; 4,81)$ & 1,000 \\
\hline neutral & Valenz & Zahnsanierung & $4,38(4,18 ; 4,59)$ & $4,76(4,59 ; 4,93)$ & 0,137 \\
\hline neutral & Arousal & Gesund & $3,27(3,02 ; 3,51)$ & $3,62(3,41 ; 3,83)$ & 0,084 \\
\hline neutral & Arousal & Karies & $3,99(3,71 ; 4,26)$ & $4,00(3,78 ; 4,22)$ & 1,000 \\
\hline neutral & Arousal & Zahnsanierung & $3,50(3,24 ; 3,76)$ & $3,61(3,40 ; 3,81)$ & 1,000 \\
\hline neutral & Attraktivität & Gesund & $4,59(4,37 ; 4,80)$ & $5,06(4,84 ; 5,28)$ & 0,041 \\
\hline neutral & Attraktivität & Karies & $4,59(4,35 ; 4,83)$ & $4,85(4,63 ; 5,07)$ & 1,000 \\
\hline neutral & Attraktivität & Zahnsanierung & $4,32(4,07 ; 4,57)$ & $4,50(4,29 ; 4,71)$ & 1,000 \\
\hline emotional & Valenz & Gesund & $3,53(3,29 ; 3,77)$ & $4,19(3,98 ; 4,40)$ & $<0,001$ \\
\hline emotional & Valenz & Karies & $4,57(4,29 ; 4,84)$ & $6,61(6,40 ; 6,83)$ & $<0,001$ \\
\hline emotional & Valenz & Zahnsanierung & $4,26(3,98 ; 4,54)$ & $5,56(5,33 ; 5,79)$ & $<0,001$ \\
\hline emotional & Arousal & Gesund & $4,59(4,32 ; 4,87)$ & $4,28(4,04 ; 4,53)$ & 1,000 \\
\hline emotional & Arousal & Karies & $5,10(4,84 ; 5,37)$ & $6,04(5,79 ; 6,29)$ & $<0,001$ \\
\hline emotional & Arousal & Zahnsanierung & $4,82(4,57 ; 5,07)$ & $5,38(5,15 ; 5,62)$ & 0,034 \\
\hline emotional & Attraktivität & Gesund & $5,62(5,38 ; 5,87)$ & $5,52(5,29 ; 5,74)$ & 1,000 \\
\hline emotional & Attraktivität & Karies & $4,47(4,23 ; 4,72)$ & $3,42(3,21 ; 3,63)$ & $<0,001$ \\
\hline emotional & Attraktivität & Zahnsanierung & $4,58(4,32 ; 4,85)$ & $4,06(3,85 ; 4,27)$ & 0,081 \\
\hline
\end{tabular}

\subsubsection{Vergleich der Gebiss-Klassifikationen}

Die zahnmedizinischen Laien beurteilten die neutralen Bilder der Kinder mit kariösen Zähnen signifikant erregender (Arousal) als die neutralen Bilder der Kinder mit gesunden Zähnen (Kruskal-Wallis-Test: $p=0,011 ; \chi^{2}=14,077$; $\mathrm{df}=2$; Nemenyi-Post-hoc-Test: $p=0,032 ; \chi^{2}$-Verteilung $=5,107$; Tabelle 5.8). Die emotionalen Bilder der Kinder mit gesunden Zähnen wurden von den Laien im Vergleich zu den Kindern mit kariösen Zähnen und mit Zahnsanierung 
signifikant angenehmer (Valenz) (Kruskal-Wallis-Test: $p<0,001 ; \chi^{2}=30,880$; $\mathrm{df}=2$; Nemenyi-Post-hoc-Test gesund vs. Karies: $\mathrm{p}<0,001 ; \chi^{2}$ Verteilung $=7,583$; Nemenyi-Post-hoc-Test gesund vs. Zahnsanierung: $p=0,019 ; \chi^{2}$-Verteilung $=5,305$ ) und attraktiver beurteilt (Kruskal-Wallis-Test: $p<0,001 ; \quad \chi^{2}=46,255 ; \quad d f=2 ;$ Nemenyi-Post-hoc-Test gesund vs. Karies $p<0,001 ; \quad \chi^{2}$-Verteilung $=8,677 ; \quad$ Nemenyi-Post-hoc-Test gesund vs. Zahnsanierung: $p<0,001 ; \chi^{2}$-Verteilung $=7,732$ ).

In Bezug auf die Bewertung der Kindergesichter durch die Experten zeigte sich, dass die neutralen Fotos der gesunden Gebisse signifikant attraktiver bewertet wurden als die Fotos der Gebisse mit Zahnsanierung (Kruskal-Wallis-Test: $p=0,012 ; \quad \chi^{2}=13,786 ; \quad d f=2 ; \quad$ Nemenyi-Post-hoc-Test: $\quad p=0,029 ; \quad \chi^{2}-$ Verteilung $=5,144)$. Die Fragen nach Valenz, Arousal und Attraktivität wurden für die emotionalen Bildtypen aller drei Gebiss-Klassifikationen durch die Experten signifikant unterschiedlich bewertet. So wurden die Kinder mit kariösen Zähnen nach Valenz am schlechtesten und für die Kinder mit gesunden Zähnen am besten bewertet (Kruskal-Wallis-Test: $p<0,001$; $\chi^{2}=183,763 ; d f=2 ;$ Nemenyi-Post-hoc-Test gesund vs. Karies: $p<0,001$; $\chi^{2}$-Verteilung $=18,934$; Nemenyi-Post-hoc-Test gesund vs. Zahnsanierung: $p<0,001 ; \quad \chi^{2}$-Verteilung $=10,646 ; \quad$ Nemenyi-Post-hoc-Test Karies vs. Zahnsanierung: $p<0,001 ; \chi^{2}$-Verteilung $\left.=8,298\right)$. Gleiches zeigte sich für Arousal: Kinder mit kariösen Zähnen wurden am erregendsten und Kinder mit gesunden Zähnen als am wenigsten erregend beurteilt (Kruskal-Wallis-Test: $p<0,001 ; \chi^{2}=87,230 ; d f=2$; Nemenyi-Post-hoc-Test gesund vs. Karies: $\mathrm{p}<0,001 ; \quad \chi^{2}$-Verteilung $=12,975 ; \quad$ Nemenyi-Post-hoc-Test gesund vs. Zahnsanierung: $\quad p=0,040 ; \quad \chi^{2}$-Verteilung $=7,946 ; \quad$ Nemenyi-Post-hoc-Test Karies vs. Zahnsanierung: $p<0,001 ; \chi^{2}$-Verteilung $\left.=5,029\right)$. Hinsichtlich der Attraktivität wurden Kinder mit gesunden Zähnen signifikant besser bewertet als Kinder mit kariösen Zähnen (Kruskal-Wallis-Test: $p<0,001 ; \chi^{2}=157,470$; $\mathrm{df}=2$; Nemenyi-Post-hoc-Test gesund vs. Karies: $p<0,001$; $\chi^{2}$-Verteilung $=17,193$; Nemenyi-Post-hoc-Test gesund vs. Zahnsanierung: $\mathrm{p}<0,001 ; \quad \chi^{2}$-Verteilung $=11,576 ; \quad$ Nemenyi-Post-hoc-Test Karies vs. Zahnsanierung: $p=0,007 ; \chi^{2}$-Verteilung $=5,629$ ). 
Tabelle 5.8: Valenz, Arousal und Attraktivität der Kinder mit gesunden Zähnen, mit kariösen Zähnen und mit Zahnsanierung beurteilt durch Laien und Experten auf neutralen und emotionalen Bildern. Die Antworten wurden als Mittelwert (MW) mit dazugehörigem 95\%-Konfidenzintervall $(\mathrm{Cl})$ angegeben. Die Gebiss-Klassifikationen für gleiche Probandinnen-Gruppe, Bildtyp und Dimension wurden mit KruskalWallis-Tests mit Nemenyi-Post-hoc-Tests verglichen. Der p-Wert wurde mit der Bonferroni-Methode korrigiert (modifiziert nach Tschammler et al. (2018), die Verwendung erfolgt mit freundlicher Genehmigung des Elsevier Verlags, Nachweis auf Seite 79).

\begin{tabular}{|c|c|c|c|c|c|c|c|c|c|}
\hline \multirow[t]{2}{*}{$\begin{array}{l}\text { Probandinnen- } \\
\text { Gruppe }\end{array}$} & \multirow[t]{2}{*}{ Bildtyp } & \multirow[t]{2}{*}{ Frage } & \multicolumn{3}{|c|}{$\begin{array}{c}\text { Gebiss- } \\
\text { Klassifizierung }\end{array}$} & \multirow[t]{2}{*}{$\begin{array}{l}\text { Korr. } \\
\text { p-Wert }\end{array}$} & \multicolumn{3}{|c|}{$\begin{array}{c}\text { Post-hoc Test } \\
\text { Korr. p-Wert }\end{array}$} \\
\hline & & & $\begin{array}{c}\text { Gesund } \\
\text { MW } \\
(\mathrm{Cl} 2,5 \% ; \mathrm{Cl} 97,5 \%)\end{array}$ & $\begin{array}{c}\text { Karies } \\
\text { MW } \\
(\mathrm{Cl} 2,5 \% ; \mathrm{Cl} 97,5 \%)\end{array}$ & $\begin{array}{c}\text { Zahnsanierung } \\
\text { MW } \\
(\mathrm{Cl} 2,5 \% ; \mathrm{Cl} 97,5 \%)\end{array}$ & & $\begin{array}{l}\text { Gesund } \\
\text { vs. } \\
\text { Karies }\end{array}$ & $\begin{array}{c}\text { Gesund } \\
\text { vs. } \\
\text { Zahnsanierung }\end{array}$ & $\begin{array}{c}\text { Karies } \\
\text { vs. } \\
\text { Zahnsanierung }\end{array}$ \\
\hline Laien & neutral & Valenz & $4,2(4,0 ; 4,4)$ & $4,4(4,1 ; 4,7)$ & $4,4(4,2 ; 4,6)$ & 1,000 & n. a. & n. a. & n. a. \\
\hline Laien & neutral & Arousal & $3,3(3,0 ; 3,5)$ & $4,0(3,7 ; 4,3)$ & $3,5(3,2 ; 3,8)$ & 0,011 & 0,032 & 1,000 & 1,000 \\
\hline Laien & neutral & Attraktivität & $4,6(4,4 ; 4,8)$ & $4,6(4,4 ; 4,8)$ & $4,3(4,1 ; 4,6)$ & 1,000 & n. a. & n. a. & n. a. \\
\hline Laien & emotional & Valenz & $3,5(3,3 ; 3,8)$ & $4,6(4,3 ; 4,8)$ & $4,3(4,0 ; 4,5)$ & $<0,001$ & $<0,001$ & 0,019 & 1,000 \\
\hline Laien & emotional & Arousal & $4,6(4,3 ; 4,9)$ & $5,1(4,8 ; 5,4)$ & $4,8(4,6 ; 5,1)$ & 0,306 & n. a. & n. a. & n. a. \\
\hline Laien & emotional & Attraktivität & $5,6(5,4 ; 5,9)$ & $4,5(4,2 ; 4,7)$ & $4,6(4,3 ; 4,9)$ & $<0,001$ & $<0,001$ & $<0,001$ & 1,000 \\
\hline Experten & neutral & Valenz & $4,5(4,3 ; 4,6)$ & $4,6(4,4 ; 4,8)$ & $4,8(4,6 ; 4,9)$ & 1,000 & n. a. & n. a. & n. a. \\
\hline Experten & neutral & Arousal & $3,6(3,4 ; 3,8)$ & $4,0(3,8 ; 4,2)$ & $3,6(3,4 ; 3,8)$ & 0,231 & n. a. & n. a. & n. a. \\
\hline Experten & neutral & Attraktivität & $5,1(4,8 ; 5,3)$ & $4,9(4,6 ; 5,1)$ & $4,5(4,3 ; 4,7)$ & 0,012 & 1,000 & 0,029 & 1,000 \\
\hline Experten & emotional & Valenz & $4,2(4,0 ; 4,4)$ & $6,6(6,4 ; 6,8)$ & $5,6(5,3 ; 5,8)$ & $<0,001$ & $<0,001$ & $<0,001$ & $<0,001$ \\
\hline Experten & emotional & Arousal & $4,3(4,0 ; 4,5)$ & $6,0(5,8 ; 6,3)$ & $5,4(5,2 ; 5,6)$ & $<0,001$ & $<0,001$ & $<0,001$ & 0,040 \\
\hline Experten & emotional & Attraktivität & $5,5(5,3 ; 5,7)$ & $3,4(3,2 ; 3,6)$ & $4,1(3,9 ; 4,3)$ & $<0,001$ & $<0,001$ & $<0,001$ & 0,007 \\
\hline
\end{tabular}




\section{Diskussion}

\subsection{Diskussion der Ergebnisse}

\subsubsection{Wahrnehmung von Kindergesichtern durch zahnmedizinische Laien und Experten}

Ein Ziel dieser Studie war es herauszufinden, ob sich zahnmedizinische Laien und Experten im Betrachtungsverhalten von Kindergesichtern mit unterschiedlichen dentalen Befunden unterscheiden.

Beide Probandinnen-Gruppen, die zahnmedizinischen Laien und die Experten, schauten mit ihrer ersten Fixation signifikant häufiger auf das emotionale Bild als auf das neutrale Bild. Dieses Verhalten offenbarte sich auch in anderen Studien, in denen die Probandinnen die emotionalen Reize ebenfalls häufiger zuerst ansahen, wenn gleichzeitig ein neutrales und ein emotionales Bild präsentiert wurden (Nummenmaa et al. 2006; Calvo und Nummenmaa 2009). Die Laien betrachteten sowohl auf den emotionalen als auch auf den neutralen Bildern signifikant häufiger das AOI Auge als die Experten. Die Experten betrachteten dafür signifikant häufiger das AOI Mund. Das Betrachtungsverhalten der Laien entspricht damit dem üblichen Verhalten, wie es bereits mehrfach beschrieben wurde (Walker-Smith et al. 1977; Hickman et al. 2010).

Die Experten fixierten am häufigsten als erstes das AOI Mund der emotionalen Bilder, respektive die Region mit sichtbaren Zähnen. Dieses Verhalten deckt sich mit den Selbstaussagen zahnmedizinischer Experten, die berichten, in Gesichtern immer zuerst die Zähne zu betrachten. Ein ähnliches Verhalten konnten Dindaroğlu et al. (2017) zeigen: Experten benötigten im Vergleich zu Laien weniger Zeit, bis die Mundregion sowohl von gesunden Patienten oder Patienten mit Lippen-Kiefer-Gaumen-Spalten fixiert wurde.

Die Experten könnten aufgrund ihrer Ausbildung ihre Aufmerksamkeit auf die Region des Gesichtes legen, welche innen die meisten Informationen über ihr Gegenüber liefert: die Mundregion. 
Die erste Alternativhypothese, dass sich die initiale Aufmerksamkeit der zahnmedizinischen Laien und Experten bei der Betrachtung von Kindergesichtern unterscheidet, wird daher bestätigt. Die erste Nullhypothese, dass sich die initiale Aufmerksamkeit bei der Betrachtung von Kindergesichtern durch zahnmedizinische Laien und Experten nicht unterscheidet, wird verworfen.

Innerhalb der ersten beiden Sekunden der Bildpräsentation wurde das emotionale Bild von beiden Probandinnen-Gruppen jeweils länger und häufiger fixiert als das neutrale Bild (Nummenmaa et al. 2006; Calvo und Nummenmaa 2009). Die Laien zeigten wieder ein signifikant größeres Interesse am AOI Auge als die Experten, die wiederum das AOI Mund länger und häufiger betrachteten. Diese Fokussierung setzte sich während der dritten Betrachtungssekunde fort. Die Experten fixierten das AOI Mund des emotionalen Bildtyps signifikant länger und häufiger als die Laien, hingegen schauten die Laien signifikant häufiger auf das $\mathrm{AOI}$ Auge der neutralen und der emotionalen Bilder. Insgesamt bekam das AOI Mund auf den emotionalen Bildern im Vergleich zu den neutralen Bildern von den Laien während der dritten Sekunde vermehrte Aufmerksamkeit, trotzdem blieb das AOI Auge für Laien das interessanteste AOI. Blanco et al. (2017) konnten ebenfalls zeigen, dass die Mundregion länger betrachtet wird, wenn Zähne sichtbar sind.

Die Alternativhypothesen zwei und drei besagen, dass sich die Betrachtung von Kindergesichtern durch Laien und Experten während der ersten beiden Sekunden bzw. der dritten Sekunde unterscheidet. Beide Alternativhypothesen werden aufgrund der angeführten Untersuchungsergebnisse angenommen. Die Nullhypothesen zwei und drei, dass sich die Betrachtung von Kindergesichtern durch Laien und Experten während der ersten beiden Sekunden bzw. der dritten Sekunde nicht unterscheidet, werden abgelehnt.

Die Sichtbarkeit der Zähne bzw. der lachende Gesichtsausdruck der emotionalen Bilder könnten ursächlich dafür sein, dass eine größere Aufmerksamkeit auf das AOI Mund (der emotionalen Bilder) gelegt wird (Calvo und Nummenmaa 2008). Der Aufmerksamkeitsfokus der Laien für das AOI Mund nimmt während der dritten Sekunde im Vergleich zu den ersten zwei 
Sekunden zu, wohingegen die Experten das AOI Mund bereits innerhalb der ersten zwei Sekunden intensiv betrachteten.

Während des klassischen Betrachtungsverhaltens von Gesichtern bekommen Augen die größte Aufmerksamkeit (Hickman et al. 2010; Eisenbarth und Alpers 2011; Richards et al. 2015). Dieses Verhalten wurde durch die Laien in der vorliegenden Studie ebenfalls gezeigt, jedoch nicht durch die Experten. Eine andere Eye-Tracking-Studie, die die Wahrnehmung von Laien und Experten verglich, konnte zeigen, dass Experten sowohl die Mundregion von Patienten mit Lippen-Kiefer-Gaumen-Spalten als auch von gesunden Patienten häufiger betrachteten als Laien (Dindaroğlu et al. 2017).

Die Ergebnisse der vorliegenden Studie lassen den möglichen Schluss zu, dass das Interessenszentrum der Experten bei der Betrachtung von Gesichtern auf die Mundregion verschoben ist. Durch das Studium und die intensive Auseinandersetzung mit Zähnen und der Mundregion lag offensichtlich eine verstärkte Aufmerksamkeit auf dem AOI Mund. Insbesondere wenn sichtbare Veränderungen, wie Karies oder therapierte Läsionen erkennbar waren, wurden diese Regionen durch die Experten länger und häufiger betrachtet. Die daraus resultierenden visuellen Abweichungen im $\mathrm{AOI}$ Mund waren offensichtlich groß genug, um für ein verändertes Betrachtungsverhalten zu sorgen.

Diese Beobachtung wird auch durch die Ergebnisse anderer Eye-TrackingStudien unterstützt. Es konnte gezeigt werden, dass Patienten, die selbst eine Lippen-Kiefer-Gaumen-Spalte (LKG-Spalte) aufwiesen, die Augenregion im Vergleich zu Probanden ohne LKG-Spalte kürzer betrachteten (Meyer-Marcotty et al. 2011; Dindaroğlu et al. 2017). Die eigene Betroffenheit scheint ebenfalls eine Verlagerung der Aufmerksamkeit bei der Betrachtung von Gesichtern auszulösen.

\subsubsection{Wahrnehmung von Kindergesichtern in Abhängigkeit vom dentalen Befund}

Die vierte Nullhypothese besagt, dass die initiale Betrachtung von Kindergesichtern nicht vom dentalen Befund (gesund, Karies und Zahnsanierung) abhängt. Als Alternativhypothese wurde formuliert, dass die initiale Betrachtung von Kindergesichtern vom dentalen Befund abhängt. 
Während der ersten Fixation der Laien wurde auf dem neutralen Bildtyp das AOI Mund der Kinder mit kariösen Zähnen signifikant häufiger angeschaut als das AOI Mund der Kinder nach Zahnsanierung. Von den Laien wurde auf dem emotionalen Bild das AOI Nase der Kinder mit gesunden Zähnen signifikant häufiger als erstes fixiert im Vergleich zu kariösen Gebissen. Von den Experten wurde auf dem neutralen Bildtyp das AOI Auge der Kinder mit gesunden Zähnen signifikant häufiger zuerst betrachtet als das der Kinder mit kariösen Zähnen. Aufgrund dieser Ergebnisse wird die vierte Alternativhypothese angenommen und die vierte Nullhypothese verworfen.

Die Vorauswahl der Präsentationsfotos sollte dafür sorgen, dass nur neutrale Bilder eingeschlossen werden, die sich hinsichtlich Valenz, Arousal und Attraktivität nicht unterscheiden. Wieso die Laien dennoch signifikante Unterschiede im Betrachtungsverhalten der neutralen Bilder für das AOI Mund zeigten und die Experten für das $\mathrm{AOI}$ Auge, lässt sich anhand der bisherigen Studienlage nicht erklären. Mutmaßlich wiesen diese AOls spezifische Auffälligkeiten auf, die nicht im Zusammenhang mit dem dentalen Befund stehen, aber dennoch zu einer anderen Betrachtung führten.

Signifikante Unterschiede im Betrachtungsverhalten der Kinder mit gesunden oder kariösen Zähnen und der Kinder mit Zahnsanierung während der ersten zwei Betrachtungssekunden zeigten lediglich die zahnmedizinischen Experten. So wurde auf den emotionalen Bildern das AOI Mund der Kinder mit kariösen Zähnen signifikant länger betrachtet als das der Kinder mit gesunden Zähnen. Ähnliches zeigte sich in anderen Studien bei der Betrachtung von Patienten mit einem großen kieferorthopädischen Behandlungsbedarf, bei innen verlagerte sich ein Großteil der visuellen Aufmerksamkeit von den Augen auf den Mund (Meyer-Marcotty et al. 2010a; Richards et al. 2015; Baker et al. 2018). Diese Studien zeigte ebenfalls, dass auffällige Gesichtsveränderungen länger und häufiger betrachtet werden. Die Mundregion von Patienten mit LKG-Spalten wurde länger und häufiger angeschaut als die Mundregion von gesunden Patienten (Meyer-Marcotty et al. 2010b; Meyer-Marcotty et al. 2011; van Schijndel et al. 2015; Dindaroğlu et al. 2017). Bei Patienten mit abstehenden Ohren wurden die Ohren länger betrachtet als bei Patienten ohne abstehende Ohren (Haworth et al. 2015; Litschel et al. 2015). 
Hinsichtlich dieser Ergebnisse wird Alternativhypothese fünf, die Wahrnehmung von Kindergesichtern hinge während der ersten beiden Sekunden vom dentalen Befund $a b$, für die Experten bestätigt und für die Laien abgelehnt. Die fünfte Nullhypothese, die Wahrnehmung von Kindergesichtern hinge während der ersten beiden Sekunden nicht vom dentalen Befund ab, wird folglich für die Experten verworfen und für die Laien angenommen.

Eine mögliche Erklärung für die intensivere Betrachtung des $\mathrm{AOI}$ Mund der emotionalen Bilder der Kinder mit kariösen Zähnen im Vergleich zu Kindern mit gesunden Zähnen könnte die Erwartungshaltung der Experten sein, in diesem Bereich innen bekannte Auffälligkeiten zu registrieren. Wenn Auffälligkeiten wie kariöse Zähne entdeckt wurden, folgte ein verstärkte Betrachtung dieser Gesichtsregion.

Für die Laien war die Ausprägung der dentalen Abweichungen eventuell nicht auffällig genug, um sie innerhalb von zwei Sekunden zu erkennen, weshalb kein verändertes Betrachtungsverhaltung in Bezug auf die unterschiedlichen dentalen Befunde stattfand. Möglicherweise sind innen die - im Vergleich zu LKG-Spalten oder abstehenden Ohren - eher kleinen Pathologien (einzelne kariöse oder fehlende Zähne) initial nicht aufgefallen und somit fand keine signifikant unterschiedliche Betrachtung statt.

Die sechste Nullhypothese sagt aus, dass die Wahrnehmung von Kindergesichtern während der dritten Sekunde nicht vom dentalen Befund abhängt. Die Alternativhypothese lautet, dass die Wahrnehmung von Kindergesichtern während der dritten Sekunde vom dentalen Befund abhängt. Die Nullhypothese wird mithilfe der vorliegenden Untersuchungsergebnisse bestätigt: Laien und Experten betrachten auch bei längerer Präsentationsdauer (dritte Betrachtungssekunde) dentale Befunde nicht unterschiedlich. Die Alternativhypothese wird entsprechend abgelehnt.

Warum beide Probandinnen-Gruppen bei längerer Betrachtungsdauer die verschiedenen dentalen Befunde nicht unterschiedlich wahrnahmen, lässt sich aufgrund fehlender vergleichbarer Studien nicht klären. Die Experten schauten zwar während der ersten beiden Sekunden das AOI Mund mit kariösen Zähnen länger an als das AOI Mund mit gesunden Zähnen. Unterschiede in der Wahrnehmung zwischen den Gebiss-Klassifizierungen gab es während der 
dritten Sekunde jedoch nicht. Die Laien betrachteten sowohl während der ersten beiden als auch während der dritten Sekunde die GebissKlassifizierungen nicht unterschiedlich.

\subsubsection{Fragebögen}

Die siebte Nullhypothese besagt, dass Laien und Experten Kindergesichter nach Valenz, Arousal und Attraktivität nicht unterschiedlich beurteilen. Als Alternativhypothese wurde aufgestellt, dass Laien und Experten Kindergesichter nach Valenz, Arousal und Attraktivität unterschiedlich beurteilen.

Die Alternativhypothese wird für die neutralen Bilder angenommen, denn die Experten bewerten die neutralen Bilder der Kinder mit gesunden Zähnen signifikant attraktiver als die Laien. Die Nullhypothese wird folglich für die neutralen Bilder verworfen. Um unterschiedliche Bewertungen der neutralen Bilder möglichst zu verhindern, wurden die neutralen Bilder in einer Vorauswahl nach Valenz, Arousal und Attraktivität beurteilt. Es wurden nur die Bilder für das Eye-Tracking-Experiment verwendet, die hinsichtlich der Bewertungen keine Auffälligkeiten aufwiesen. Für die Vortestungen wurden Zahnärztinnen und Zahnärzte befragt. Möglicherweise ähnelten sie mit ihren Bewertungen stärker den Experten als den Laien. Mithilfe eines gemischten Kollektivs aus Laien und Experten wären in den Vortestungen vielleicht andere Bilder ausgewählt worden.

Hinsichtlich der Bewertung der emotionalen Bilder zeigten sich diverse Unterschiede zwischen den Probandinnen-Gruppen. Die Experten bewerteten alle drei Gebiss-Klassifizierungen nach Valenz signifikant weniger positiv als die Laien. Die Kinder mit kariösen Zähnen und die Kinder mit Zahnsanierung wurden durch die Experten signifikant erregender (Arousal) bewertet als durch die Laien, in der Bewertung der Attraktivität wurden die Kinder mit kariösen Zähnen von den Experten signifikant unattraktiver bewertet als von den Laien. Aufgrund der vorliegenden Ergebnisse wird die siebte Alternativhypothese, die Unterschiede in der Beurteilung der Probandinnen-Gruppen annahm, für die emotionalen Bilder bestätigt. Die Nullhypothese, die keine Unterschiede erwartete, wird für die emotionalen Bilder abgelehnt. 
Die dargelegten Beobachtungen decken sich mit den Ergebnissen anderer Studien zur Wahrnehmung und Attraktivität von Gesichtsveränderungen durch zahnmedizinische Laien und Experten (Kokich et al. 1999; Kokich et al. 2006; Dindaroğlu et al. 2017). Diese Studien konnten nachweisen, dass Experten dentale und kraniale Veränderungen bereits bei geringem Ausprägungsgrad registrieren und diese als störend empfinden. Im Gegensatz dazu nahmen Laien erst größere Abweichungen wahr. Oosterhaven et al. (1989) zeigten, dass bei Erwachsenen das Fehlen eines Frontzahnes eine besonders große Veränderung darstellt, die von Experten und Laien gleichermaßen erkannt wird und zu ähnlich negativen Attraktivitätsbewertungen führt.

Die Laien bewerteten alle emotionalen Gesichter hinsichtlich Valenz positiver als die Experten. Gründe dafür könnten sein, dass die Laien die Kindergesichter grundsätzlich niedlicher empfanden und die Experten im Gegensatz dazu eine rationalere Herangehensweise an die Fragestellung zeigten.

Dass Kinder mit kariösen Zähnen und nach Zahnsanierung durch die Experten signifikant erregender bewerten wurden, könnte am Wissen um die Erkrankung Karies liegen. Gleiches gilt für die schlechtere Attraktivitätsbewertung der kariösen Zähne durch die Experten. Darüber hinaus wäre denkbar, dass die Experten eine Erwartungshaltung hinsichtlich eines idealen Gebisses haben, die nur durch die gesunden Kinder erfüllt wurde, nicht aber durch kariöse oder therapierte Zähne. Alle Abweichungen von dieser Norm könnten als fehlerhaft interpretiert und entsprechend bewertet worden sein. Das Abweichen von der Idealvorstellung könnte potenziell zu einer sozialen Ausgrenzung dieser Kinder führen. Eine solche Stigmatisierung der Kinder aufgrund nicht perfekter Gebisse mit beispielsweise kariösen Zähnen konnte durch einige Autoren bereits aufzeigen werden (Shaw 1981; Filstrup et al. 2003).

Die Laien scheinen diese Abgrenzung in der Bewertung in der vorliegenden Studie nicht vorzunehmen. Wahrscheinlich hatten sie bezüglich der gezeigten Gebisse keine Erwartungshaltung oder innen waren die schädlichen Folgen kariöser Milchzähne wie beispielsweise die vollständige Zerstörung der Zahnkrone oder die Schädigung der bleibenden Zähne nicht bewusst.

Fotografien von Kindern mit sichtbarer Karies und nach Zahnsanierung (Füllungen, Stahlkronen und/oder Lücken nach Zahnextraktionen) wurden 
sowohl von den zahnärztlichen Experten als auch von den Laien als signifikant unattraktiver bewertet als Kinder mit gesunden Zähnen. Dass gesunde Gebisse sowohl von Kindern als auch von Erwachsenen im Vergleich zu pathologisch veränderten Gebissen attraktiver bewertet wurden, konnten andere Autoren ebenfalls zeigen (Shaw 1981; Karunakaran et al. 2011).

Im Vergleich der Gebiss-Klassifizierungen gegeneinander zeigte sich, dass nur die Experten eine weitere Abstufung vornahmen: Kinder mit kariösen Zähnen wurden jeweils als unangenehmer (Valenz), erregender (Arousal) und unattraktiver (Attraktivität) bewertet als Kinder mit Zahnsanierung. Alternativhypothese acht wird daher für beide Probandinnen-Gruppen angenommen: dentale Befunde haben Einfluss auf die Bewertung von Kindergesichtern nach Valenz, Arousal und Attraktivität. Die achte Nullhypothese, dass dentale Befunde die Bewertung von Kindergesichtern nach Valenz, Arousal und Attraktivität nicht beeinflussen, wird für Laien und Experten verworfen.

Die Abstufung der emotionalen Bilder in der Bewertung aller drei Fragen durch die Experten in der Reihenfolge: gesund > Zahnsanierung > Karies könnte mit der bereits erwähnten Erwartungshaltung zusammenhängen. Gesunde Zähne sind wahrscheinlich der gewünschte Idealzustand, gefolgt von sanierten Gebissen und kariösen Zähnen.

Die Laien zeigten im Betrachtungsverhalten der verschiedenen dentalen Befunde keine signifikanten Unterschiede, bei der Bewertung hingegen schon. Daraus ergibt sich die Frage, wodurch sie hervorgerufen wurden. Ein möglicher Erklärungsversuch könnte sein, dass bei der Bewertung der Bilder jedes Bild einzeln gezeigt wurde und pro Bild mehr Zeit zur Betrachtung zur Verfügung stand im Vergleich zum Eye-Tracking-Experiment. Auch könnten die Auswertungskriterien der Eye-Tracking-Untersuchung hinsichtlich erster Fixation, Fixationszeit und Fixationsanzahl nicht sensitiv genug gewesen sein, um Abweichungen aufzuzeigen. Eine weiterführende Analyse der Daten unter Auswertung der Sakkaden könnte dies eventuell aufzeigen oder in einer weiterführenden Eye-Tracking-Studie mit länger Präsentationsdauer der Bilder untersucht werden. 


\subsection{Diskussion der Methode}

\subsubsection{Fotos der Patienten und Auswahl der Fotos}

Für die vorliegende Studie wurden weibliche und männliche Kinder im Alter von zwei bis zehn Jahren fotografiert (Coutrot et al. 2016). Die Fotos wurden unter standardisierten Bedingungen aufgenommen. Allerdings mussten einige Bilder ausgeschlossen werden, weil besonders sehr junge Kinder nicht ausreichend ruhig sitzen konnten, so dass die Bilder unscharf oder verwackelt waren. Danach wurden die Gesichter hinsichtlich der Gesichtsmitte und des Pupillenabstandes ausgerichtet. Alle Bereiche außerhalb des Gesichtes wie Haare, Ohren oder Hintergrund wurden mit einem mittleren Grau überzeichnet, um eine Ablenkung durch diese Bereiche zu vermeiden. Dies machten sich bereits einige Studien zunutze (Meyer-Marcotty et al. 2010b; Dindaroğlu et al. 2017), andere hingegen verzichteten auf die Reduzierung des Hintergrundes (van Schijndel et al. 2015). Durch die Reduktion der Bilder auf die nötigsten Strukturen und die standardisierten Aufnahmebedingen wurde eine hohe Vergleichbarkeit der Bilder in Bezug auf Kontrast und Helligkeit der Bilder erzielt (Sheth und Pham 2008). Nichtsdestoweniger können eventuelle Auswirkungen von Gesichtsmerkmalen oder Ethnie auf die Wahrnehmung der Gesichter nicht ausgeschlossen werden (Meissner und Brigham 2001). Bei den präsentierten Bildern handelte es sich um Farbfotos. Zwar wurden bereits andere vergleichbare Experimente mit schwarz-weiß Bildern durchgeführt (MeyerMarcotty et al. 2011; Linz et al. 2016), jedoch scheint für eine korrekte Interpretation von Gesichtern die Farbe eine nicht unerhebliche Rolle zu spielen (Tan und Stephen 2013). Zudem ist es deutlich schwieriger, dentale Befunde zu erkennen, wenn die Farbinformationen der Bilder fehlen, weshalb für die vorliegende Studie auf Farbfotos zurückgegriffen wurde.

Die Entscheidung, welche Patientenfotos in die Studie eingeschlossen wurden, wurde anhand einer Vorbewertung der Fotos nach Valenz, Arousal und Attraktivität getroffen. Die Vorbewertung der Fotos hinsichtlich ihrer Attraktivität fand in Anlehnung an frühere Studien statt (Karunakaran et al. 2011; Dindaroğlu et al. 2017). Die Reliabilität dieser Bewertung konnte in früheren Studien nachgewiesen werden (Howells und Shaw 1985). Daneben empfiehlt Russell (1980) für die Bewertung von Emotionen zudem sein circumplex model mit den 
Parametern Valenz und Arousal. Andere Wahrnehmungsstudien nutzten dieses Model ebenfalls und konnten seine Reproduzierbarkeit nachweisen (Alpers und Gerdes 2007; Adolph und Alpers 2010; Eisenbarth und Alpers 2011).

Anschließend erfolgte eine Eingruppierung der Fotos in die drei Gebiss-Klassifizierungen „gesund“, „Karies“ und „Zahnsanierung“. Die Kinder mit gesunden Zähnen dienten als Referenz, um eventuelle Unterschiede zu Kindern mit Karies oder mit therapierter Karies zu untersuchen. Wahrscheinlich litt ein Großteil der Kinder, deren Zähne als kariös oder als saniert klassifiziert wurden, an einer frühkindlichen Karies. Diese Erkrankung tritt definitionsgemäß aber nur bei Kindern unter sechs Jahren auf (Wyne 1999), so dass für ältere Kinder diese Diagnose in der Rückschau nur vermutet, aber nicht als gesichert angenommen werden kann.

Um eine belastbare Einteilung der Patienten hinsichtlich der drei GebissKlassifizierungen zu erhalten, erfolgte diese durch 15 erfahrene Zahnärztinnen und Zahnärzte (Alter in Jahren 31,0 $\pm 9,7$ ). Eine Zuordnung anhand von klinischen Untersuchungen oder Parametern, wie dies üblicherweise in Eye-Tracking-Studien über Gesichtsveränderungen durchgeführt wird, war nicht möglich (Meyer-Marcotty et al. 2011; van Schijndel et al. 2015; Dindaroğlu et al. 2017), da zwar für gesunde und kariöse Zähne standardisierte zahnmedizinische Indices existieren, nicht jedoch für die gewählte Kategorie „Zahnsanierung“. Die Einordnung der Fotografien hatte zudem den Vorteil, dass ausschließlich eindeutig erkennbare Fälle eingeschlossen wurden (nötige Interrater-Übereinstimmung $\geq 60 \%$ ). Zudem konnte eine gleichmäßige Geschlechter- und Altersverteilung in den drei Gebiss-Klassifikationen erreicht werden.

\subsubsection{Durchführung der Eye-Tracking-Untersuchung}

\subsubsection{Probandinnen}

Für den Ein- und Ausschluss der Eye-Tracking-Probandinnen wurden diverse Kriterien definiert. Es wurden nur weibliche Rechtshänder eingeschlossen, die keine schweren allgemeinen oder psychischen Erkrankungen aufwiesen und keine Drogen oder Medikamente einnahmen, welche zu einer veränderten Wahrnehmung oder neurophysiologischen Reizverarbeitung führen könnten. 
Diese Kriterien basierten auf den Ergebnissen vorangegangener Studien (Bowers und Heilman 1980; Moser et al. 1998; Pelphrey et al. 2002; Vassallo et al. 2009; Adolph und Alpers 2010; Hall et al. 2010; Huestegge et al. 2010; Coutrot et al. 2016; Nicholls et al. 2017; Silva et al. 2017). Ziel war es, ein möglichst homogenes und vergleichbares Probandinnenkollektiv mit einer größtmöglichen Reduktion individueller Einflussfaktoren zu gewinnen. Aus technischen Gründen mussten zudem Brillenträger ausgeschlossen werden, da das verwendete Eye-Tracking-System die dark-pupil-Methode nutzte. Dies detektiert die Lichtbrechung eines Infrarotlichtstrahles durch das Auge und kann von spiegelnden Brillengläsern verändert werden (SensoMotoric Instruments $\mathrm{GmbH}$ 2014). Da es sich bei den beiden Gruppen um sehr ausgewählte Probandinnen handelte, können die Ergebnisse nicht uneingeschränkt auf die Allgemeinbevölkerung und alle Zahnärzte übertragen werden.

Die Probandinnen wurden erst nach dem Experiment vollumfänglich über die Ziele der Studie informiert. Trotzdem ist es möglich, dass die Probandinnen während der Durchführung der Studie erkannt haben, dass die Studie auf Unterschiede hinsichtlich der Wahrnehmung besonderer dentaler Befunde abzielte. Dieser Problematik hätte möglicherweise mit einer höheren Anzahl an Fotos von gesunden Kindern entgegengesteuert werden können. Eine höhere Anzahl an Präsentationsbildpaaren hätte wiederum das Experiment verlängert, womit die Konzentrationsfähigkeit der Probandinnen und die Validität der Ergebnisse möglicherweise herabgesetzt worden wäre.

\subsubsection{Eye-Tracking-Experiment}

Die Untersuchung wurde mit dem Eye-Tracking-Systems iView $X^{\text {TM }}$ Hi-Speed 1250 (SensoMotoric Instruments $\mathrm{GmbH}$, Teltow) und der Software iView $\mathrm{X}^{\mathrm{TM}}$ (SensoMotoric Instruments $\mathrm{GmbH}$, Teltow) durchgeführt. Es wurden die Bewegungen des linken Auges aufgezeichnet. Vergleichbare Wahrnehmungsstudien nutzten technisch ähnliche Geräte und zeichneten ebenfalls die Augenbewegungen monokular auf (Meyer-Marcotty et al. 2011). Es kann davon ausgegangen werden, dass beide Augen die gleichen Ziele fixieren, da sogenannte Vergenzbewegungen dazu führen, dass die Fixationen beider Augen synchronisiert werden (Hendriks 1996; Liversedge et al. 2006). 
Während der Untersuchung erschienen immer im Wechsel für 1,5 Sekunden ein weißes Fixationskreuz, dann für 3,0 Sekunden ein Präsentationsbildpaar und anschließend für 1,5 Sekunden ein grauer Bildschirm. Ein Präsentationsbildpaar zeigte nebeneinander ein neutrales und ein emotionales Foto eines Kindes. Insgesamt wurden die Fotopaare von 39 Kindern eingeschlossen, die jeweils einmal mit dem neutralen Bild links und einmal mit dem neutralen Bild rechts präsentiert wurden. Das emotionale Bild wurde entsprechend auf der anderen Seite dargeboten. Die zufällige Präsentation eines neutralen und eines emotionalen Bildes auf der linken bzw. rechten Seite und die randomisierte Reihenfolge der Präsentationsbilder erfolgte, um eine gezielte Betrachtung einer Seite zu verhindern.

Die Probandinnen wurden instruiert das Fixationskreuz vor jedem Präsentationsbild anzuschauen. Das weiße Fixationskreuz diente dazu, den Blick vor jedem Präsentationsbild erneut mittig zu platzieren. Auf einem separaten Kontrollbildschirm wurde durch den Versuchsleiter während des Experimentes eine Fixationskontrolle durchgeführt und die Lokalisation der aktuellen Fixation überwacht. So wurde sichergestellt, dass ein initialer Sakkadensprung zu einem der beiden Bilder nötig war. Insbesondere für die Analyse der ersten Fixation ist dies von essenzieller Bedeutung (Nummenmaa et al. 2006; Calvo und Nummenmaa 2009). Der graue Bildschirm als Interstimulusintervall nach jedem Präsentationsbild diente als kurze Reizunterbrechung (Dorsch 2017a).

Für alle Fotos wurden jeweils die AOls Auge, Nase und Mund definiert (Linz et al. 2016; Dindaroğlu et al. 2017). Ausgewertet wurde das Betrachtungsverhalten während der drei Sekunden, in denen das Präsentationsbild gezeigt wurde. Die Analyse der drei Sekunden wurde aufgeteilt in die ersten zwei Sekunden und die dritte Sekunde. Diese Aufteilung erfolgte, da zwei Sekunden benötigt werden, um ein normales Bild vollständig wahrzunehmen (Kroeber-Riel 2001; Kroeber-Riel et al. 2015). Mit längerer Beobachtungszeit wird das Betrachtungs- und Wahrnehmungsverhalten hingegen zunehmend von emotionalen Einflüssen oder durch bewusste Wahrnehmungsprozesse moduliert, was sich in einem veränderten Betrachtungsverhalten bemerkbar machen kann (Steinfurth et al. 2013). Daraus resultierte die Annahme, dass in der dritten Sekunde interessante oder 
hervorstechende Bereiche vermehrt betrachtet würden, was die vorliegende Studie jedoch nicht nachweisen konnte. Die erste Fixation wurde wegen ihrer großen Bedeutung für die initiale Aufmerksamkeit gesondert ausgewertet (Nummenmaa et al. 2006; Calvo und Nummenmaa 2009).

\subsection{Schlussfolgerungen}

Im Rahmen dieser Studie konnte gezeigt werden, dass zahnmedizinische Laien Fotoaufnahmen von Kindergesichtern mit und ohne besondere dentale Auffälligkeiten innerhalb der ersten Sekunden eines Eye-Tracking-Experiments anders betrachten und wahrnehmen als zahnmedizinische Experten. Die zahnmedizinischen Laien zeigten während der Eye-Tracking-Untersuchung größeres Interesse an der Augenregion. Wohingegen die Hauptbetrachtung der zahnmedizinischen Experten auf der Mundregion lag.

Kinder mit sichtbaren kariösen Zähnen oder nach umfangreicher Zahnsanierung (Füllungen, Stahlkronen und/oder Extraktionen) wurden sowohl von Laien als auch von Experten hinsichtlich Valenz, Arousal und Attraktivität signifikant negativer bewertet als Kinder mit gesunden Gebissen, was auf eine mögliche Erwartungshaltung hinsichtlich des Aussehens hindeutet. Diese Ergebnisse bestätigen eingeschränkt die Beobachtungen vorhergegangener Studien, dass Kinder mit Karies von Erwachsenen negativer wahrgenommen werden als Kinder mit gesunden Zähnen und dadurch vermehrt soziale Ablehnung erfahren (Shaw 1981; Filstrup et al. 2003; Somani et al. 2010).

Bei nur kurzzeitiger Betrachtung (Eye-Tracking-Experiment) war der Aufmerksamkeitsfokus der Laien nicht auf die Mundregion verschoben. Bei zeitlich unbegrenzter Betrachtung der Fotos und Bewertung hinsichtlich Valenz, Arousal und Attraktivität wurden die Kinder mit kariösen Zähnen jedoch schlechter bewertet. Darüber hinaus konnte die vorliegende Untersuchung zeigen, dass nicht nur Kinder mit kariösen Läsionen, sondern auch Kinder nach umfangreicher Zahnsanierung anders wahrgenommen werden. Die Ergebnisse lassen darauf schließen, dass bei Kindern bereits farblich-strukturelle Abweichungen (Karies/Stahlkronen) zu einer veränderten Betrachtung, Wahrnehmung und Bewertung der Gesichter führen. Dieser Unterschied 
manifestierte sich noch nicht in den ersten Sekunden, sondern erst in der späteren Betrachtungsphase.

Die Erkenntnisse der vorliegenden Studie unterstreichen die Bedeutung prophylaktischer Maßnahmen und den frühen Therapiebeginn beim Auftreten kariöser Läsionen im frühen Kindesalter, nicht nur aufgrund der medizinischen Folgen, sondern auch zur Vermeidung einer potenziellen sozialen Ausgrenzung des Kindes.

\subsection{Ausblick}

Im Rahmen dieser Studie konnte die unterschiedliche Wahrnehmung von Erwachsenen bei der Betrachtung von Kindergesichtern mit verschiedenen dentalen Befunden aufgezeigt werden, ebenso wie die schlechtere Bewertung von erkrankten Kindern mit Karies und nach entsprechender Therapie in Bezug auf Valenz, Arousal und Attraktivität. Als Probanden dienten dabei adulte, weibliche Rechtshänder. Daran anschließend stellt sich die Frage, ob ein anderes Probandenkollektiv die präsentierten Kindergesichter (mit gesunden Zähnen, Karies und nach Zahnsanierung) anders wahrnehmen und unterschiedlich bewerten würde. Interessant wäre zum Beispiel die Wahrnehmung der Kinder durch gleichaltrige Kinder oder gemischtgeschlechtliche Erwachsene zu untersuchen. Außerdem wäre es sicher aufschlussreich mithilfe anderer Fragebögen die Wahrnehmung von Kindern auf die unterschiedlichen Patientengruppen (gesund, Karies und Zustand nach umfangreicher Zahnsanierung) zu untersuchen und dabei insbesondere die eigene Karieserfahrung der Kinder zu berücksichtigen. Dies wird aktuell in einer neuen Studie der Poliklinik für Präventive Zahnmedizin, Parodontologie und Kariologie der Universitätsmedizin Göttingen untersucht, die Kinder im Alter von vier bis neun Jahren und ihre Eltern befragt.

Neben der Auswertung der Eye-Tracking-Untersuchung hinsichtlich erster Fixation, Fixationszeit und Fixationsanzahl könnten zukünftige Studien zudem um andere Augenbewegungsparameter ergänzt werden wie beispielsweise der Auswertung von Sakkaden. Auch wären weitere Studien mit längerer Präsentationsdauer der Bilder denkbar, um das bewusste Betrachtungsverhalten von Probanden noch deutlicher untersuchen zu können. 


\section{Zusammenfassung}

Ziel der Studie: Ziel dieser Untersuchung war es, die Wahrnehmung von zahnmedizinischen Laien und Experten zu vergleichen und zu ermitteln, ob Kinder mit sichtbarer Karies anders betrachtet werden als Kinder mit gesunden Gebissen oder nach zahnärztlicher Behandlung mit Füllungen, Zahnextraktion und/oder Stahlkronen.

Material und Methoden: Im Rahmen der Eye-Tracking-Studie wurden videobasiert die Blickbewegungen von weiblichen, zahnmedizinischen Laien $(n=20)$ und weiblichen, zahnmedizinischen Experten $(n=18)$ aufgezeichnet, während innen Bildpaare von Kindergesichtern (Frontalaufnahmen mit geschlossenem bzw. lachendem Mund) präsentiert wurden. Die Bildpaare stammten von 39 Kindern im Alter von zwei bis zehn Jahren mit gesunden Zähnen ( $n=13)$, sichtbaren kariösen Zähnen $(n=13)$ und nach Zahnsanierung (Füllungen, Extraktionen und/oder Stahlkronen) $(n=13)$. Von jedem Kind wurden zwei Bildpaare in randomisierter Reihenfolge präsentiert: (1) lachend links und geschlossener Mund rechts, (2) geschlossener Mund links und lachend rechts. Es wurde die Verteilung der ersten Fixation und das Betrachtungsverhalten (Gesamtfixationszeiten und Fixationsanzahl) in den areas of interest (AOI) Auge, Nase und Mund in den ersten beiden Sekunden und der dritten Sekunde der Präsentation ausgewertet. Nach der Eye-Tracking-Untersuchung erfolgte eine Bewertung jedes einzelnen Fotos durch die Probandinnen hinsichtlich der Kriterien Valenz, Arousal und Attraktivität. Die statistische Auswertung erfolgte mittels Binomial-, Mann-Whitney-U- und Kruskal-Wallis-Tests $(p<0,05)$.

Ergebnisse: Zahnmedizinische Laien betrachteten sowohl mit der ersten Fixation, in der ersten und zweiten Sekunde, als auch in der dritten Sekunde die Augenregion länger und häufiger als Experten, die signifikant länger und häufiger die Mundregion fixierten. Die Mundregion von Kindern mit sichtbarer Karies wurde von den Experten während der ersten beiden Sekunden signifikant länger fixiert als die Mundregion von Kindern mit gesunden Zähnen.

Die Bilder von Kindern mit kariösen Zähnen wurden von den Laien und Experten als unangenehmer, erregender und unattraktiver bewertet als Kinder mit gesunden Zähnen und nach Zahnsanierung. 
Schlussfolgerungen: Kinder mit sichtbaren Zähnen, die Karies oder Zahnsanierungen aufwiesen, wurden durch Laien und Experten anders wahrgenommen als Kinder mit gesunden Zähnen. Zahnmedizinische Experten nahmen Karies anders wahr als Laien und betrachteten die Mundregion intensiver als Laien, die die Augenregion stärker anschauten. Sowohl Laien als auch Experten bewerteten Kinder mit sichtbarer Karies und Zahnsanierung negativer als Kinder mit gesunden Zähnen. 


\section{Literaturverzeichnis}

Acs G, Lodolini G, Kaminsky S, Cisneros GJ (1992): Effect of nursing caries on body weight in a pediatric population. Pediatr Dent 14, 302-305

Adolph D, Alpers GW (2010): Valence and Arousal: A Comparison of Two Sets of Emotional Facial Expressions. Am J Psychol 123, 209-219

Agostini FG, Flaitz CM, Hicks MJ (2001): Dental emergencies in a universitybased pediatric dentistry postgraduate outpatient clinic: a retrospective study. ASDC J Dent Child $\underline{68}$, 316-321

Alm A, Wendt LK, Koch G, Birkhed D (2007): Oral Hygiene and Parent-Related Factors during Early Childhood in Relation to Approximal Caries at 15 Years of Age. Caries Res $\underline{42}, 28-36$

Alpers GW, Gerdes ABM (2007): Here is looking at you: emotional faces predominate in binocular rivalry. Emotion $\underline{7}, 495-506$

American Academy of Pediatric Dentistry (2003): Definition of Early Childhood Caries (ECC). www.aapd.org/assets/1/7/D_ECC.pdf; Zugriff am 23.03.2016

American Academy of Pediatric Dentistry (2016): Guideline on Infant Oral Health Care. Pediatr Dent 37, 146-150

American Dental Association Council on Scientific Affairs (2014): Fluoride toothpaste use for young children. J Am Dent Assoc 145, 190-191

Ando M, van der Veen MH, Schemehorn BR, Stookey GK (2001): Comparative Study to Quantify Demineralized Enamel in Deciduous and Permanent Teeth Using Laser- and Light-Induced Fluorescence Techniques. Caries Res $\underline{35}, 464-470$

Antes JR, Penland JG: Picture Context Effects on Eye Movement Patterns. In: Fisher DF, Monty RA, Senders JW (Hrsg.): Eye movements: cognition and visual perception. Lawrence Erlbaum Associates, Hillsdale 1981, 157-170

Arnim S, Doyle M (1959): Dentin dimensions of primary teeth. J Dent Child 26, 191-214

Babeely K, Kaste LM, Husain J, Behbehani J, al-Za'abi F, Maher TC, Tavares M, Soparkar P, DePaola P (1989): Severity of nursing-bottle syndrome and feeding patterns in Kuwait. Community Dent Oral Epidemiol 17, 237-239

Baker RS, Fields HW, Beck FM, Firestone AR, Rosenstiel SF (2018): Objective assessment of the contribution of dental esthetics and facial attractiveness in men via eye tracking. Am J Orthod Dentofacial Orthop 153, 523-533

BaniHani A, Deery C, Toumba J, Munyombwe T, Duggal M (2018): The impact of dental caries and its treatment by conventional or biological approaches on the oral health-related quality of life of children and carers. Int $\mathrm{J}$ Paediatr Dent 28, 266-276

Bekes K (2017): Frühkindliche Karies - Prävalenz, Risikofaktoren und Präventionsansätze. Dtsch Zahnärztl Z 126, 552-558

Bellebaum C, Thoma P, Daum I: Neuropsychologie. VS Verlag für Sozialwissenschaften, Wiesbaden 2012, 31-46 
Bissar A, Schiller P, Wolff A, Niekusch U, Schulte AG (2014): Factors contributing to severe early childhood caries in south-west Germany. Clin Oral Investig 18, 1411-1418

Blanco I, Serrano-Pedraza I, Vazquez C (2017): Don't look at my teeth when I smile: Teeth visibility in smiling faces affects emotionality ratings and gaze patterns. Emotion 17, 640-647

Boff KR, Lincoln JE: Engineering Data Compendium. Human Perception and Performance. Band 1; o. Verl., Dayton 1988

Borutta A, Kneist S, Kischka P, Eherler D, Chemnitius P, Stösser L (2002): Die Mundgesundheit von Kleinkindern in Beziehung zu relevanten Einflussfaktoren. Dtsch Zahnärztl Z 푸, 682-687

Boumans LJJM, Rodenburg M, Maas AJJ (1980): Statistical Evaluation of Nystagmus in Cupulometry. ORL J Otorhinolaryngol Relat Spec 42, 292303

Bowers D, Heilman KM (1980): Pseudoneglect: Effects of hemispace on a tactile line bisection task. Neuropsychologia $\underline{18}$, 491-498

Bradley MM, Lang PJ (1994): Measuring emotion: the self-assessment manikin and the semantic differential. J Behav Ther Exp Psychiatry 25, 49-59

Brainard DH (1997): The Psychophysics Toolbox. Spat Vis $\underline{10}$, 433-436

Brockhaus' kleines Konversationslexikon: Eintrag Sakkade. Band 1, 5. Auflage; Brockhaus, Leipzig 2000

Brown WE, Gregory TM, Chow LC (1977): Effects of Fluoride on Enamel Solubility and Cariostasis. Caries Res $\underline{11}, 118-141$

Buswell GT: How people look at pictures: a study of the psychology and perception in art. University of Chicago Press, Chicago 1935

Calvo MG, Nummenmaa L (2008): Detection of emotional faces: salient physical features guide effective visual search. J Exp Psychol Gen 137, 471-494

Calvo MG, Nummenmaa L (2009): Eye-movement assessment of the time course in facial expression recognition: Neurophysiological implications. Cogn Affect Behav Neurosci $\underline{9}$, 398-411

Carpenter RHS: Movements of the eyes. Pion, London 1977

Carrasco M (2011): Visual attention: The past 25 years. Vision Res $\underline{51}$, 14841525

Casamassimo PS, Thikkurissy S, Edelstein BL, Maiorini E (2009): Beyond the $\mathrm{dmft}$ : the human and economic cost of early childhood caries. J Am Dent Assoc 140, 650-657

Christoffersen MR, Christoffersen J, Arends J (1984): Kinetics of dissolution of calcium hydroxyapatite. J Cryst Growth 67, 107-114

Cole AS, Eastoe JE: Biochemistry and Oral Biology. 2. Auflage; Elsevier Science, London 2014

Coutrot A, Binetti N, Harrison C, Mareschal I, Johnston A (2016): Face exploration dynamics differentiate men and women. $\mathrm{J}$ Vis $\underline{16}, 16$ 
Crane HD, Steele CM (1978): Accurate three-dimensional eyetracker. Applied Optics $\underline{17}, 691$

Davies GN (1998): Early childhood caries - a synopsis. Community Dent Oral Epidemiol 26, 106-116

Dawes $\mathrm{C}$ (2003): What is the critical $\mathrm{pH}$ and why does a tooth dissolve in acid? J Can Dent Assoc 69, 722-724

De Menezes Oliveira MAH, Torres CP, Gomes-Silva JM, Chinelatti MA, De Menezes FCH, Palma-Dibb RG, Borsatto MC (2009): Microstructure and mineral composition of dental enamel of permanent and deciduous teeth. Microsc Res Tech $\underline{73}, 572-577$

Dean HT, Jay P, Arnold Jr FA, Elvove E (1941): Domestic water and dental caries: II. a study of 2,832 white children, aged 12-14 years, of 8 suburban chicago communities, including lactobacillus acidophilus studies of 1,761 children. Public Health Rep $\underline{56}, 761-792$

Dean HT, Arnold FA, Elvove E (1942): Domestic water and dental caries: V. Additional studies of the relation of fluoride domestic waters to dental caries experience in 4,425 white children, aged 12 to 14 years, of 13 cities in 4 states. Public Health Rep $\underline{57}, 1155-1179$

Deichsel M, Rojas G, Lüdecke K, Heinrich-Weltzien R (2012): Frühkindliche Karies und assoziierte Risikofaktoren bei Kleinkindern im Land Brandenburg. Bundesgesundheitsbl $\underline{55}$, 1504-1511

de Leeuw NH (2004): Resisting the Onset of Hydroxyapatite Dissolution through the Incorporation of Fluoride. J Phys Chem B $\underline{108}$, 1809-1811

Deutsche Arbeitsgemeinschaft für Jugendzahnpflege e. V. (2016): Frühkindliche Karies: zentrale Inhalte der Gruppenprophylaxe für unter 3jährige Kinder: Erweiterte Empfehlungen der Deutschen Arbeitsgemeinschaft für Jugendzahnpflege e. V. http://www.daj.de/fileadmin/user_upload/PDF_Downloads/PM_Empfehlung en_Expertise_2016/Kurzversion_DAJ_Empf2016.pdf; Zügriff am 16.03.2018

Deutsche Arbeitsgemeinschaft für Jugendzahnpflege e. V.: Epidemiologische Begleituntersuchungen zur Gruppenprophylaxe 2016. o. Verl., Bonn 2017

Deutsche Gesellschaft für Zahn-, Mund- und Kieferheilkunde (2013): S2kLeitlinie: Fluoridierungsmaßnahmen zur Kariesprophylaxe. www.dgzmk.de/uploads/tx_szdgzmkdocuments/LLFluoridierungLangUpdat e2013.pdf; Zugriff am 15.02.2018

Dewhirst FE, Chen T, Izard J, Paster BJ, Tanner ACR, Yu W-H, Lakshmanan A, Wade WG (2010): The Human Oral Microbiome. J Bacteriol 192, 50025017

Dindaroğlu F, Doğan S, Amado S, Doğan E (2017): Visual perception of faces with unilateral and bilateral cleft lip and palate: An Eye-Tracking Study. Orthod Craniofac Res 20, 44-54

Dodge R (1907): An experimental study of visual fixation. Psychol Monogr $\underline{8}, 1-$ 95 
Dorsch - Lexikon der Psychologie: Eintrag Interstimulusintervall (ISI). 18. Auflage; Hogrefe, Bern 2017a

Dorsch - Lexikon der Psychologie: Eintrag Valenz. 18. Auflage; Hogrefe, Bern $2017 b$

Duchowski A: Eye Tracking Methodology. 2. Auflage; Springer, London 2007

Duncan J, Humphreys GW (1989): Visual search and stimulus similarity. Psychol Rev 96, 433-458

Eisenbarth H, Alpers GW (2011): Happy mouth and sad eyes: Scanning emotional facial expressions. Emotion 11, 860-865

Fass E (1962): Is bottle feeding of milk a factor in dental caries? J Dent Child $\underline{29}, 245-251$

Featherstone JDB, Mellberg JR (1981): Relative Rates of Progress of Artificial Carious Lesions in Bovine, Ovine and Human Enamel. Caries Res $\underline{15}, 109$ 114

Fejerskov O (2004): Changing Paradigms in Concepts on Dental Caries: Consequences for Oral Health Care. Caries Res $\underline{38}$, 182-191

Fejerskov O: Pathology of dental caries. In: Fejerskov O, Nyvad B, Kidd EAM (Hrsg.): Dental caries: the disease and its clinical management. 3. Auflage; Wiley Blackwell, Oxford 2015, 49-81

Fejerskov O, Cury JA, Tenuta LM, Marinho VC: Fluorides in caries control. In: Fejerskov O, Nyvad B, Kidd EAM (Hrsg.): Dental caries: the disease and its clinical management. 3. Auflage; Wiley Blackwell, Oxford 2015, 245-276

Filstrup SL, Briskie D, da Fonseca M, Lawrence L, Wandera A, Inglehart MR (2003): Early childhood caries and quality of life: child and parent perspectives. Pediatr Dent $\underline{25}, 431-440$

Findlay JM, Gilchrist ID: Eye Guidance and Visual Search; In: Underwood G (Hrsg.): Eye guidance in reading and scene perception. Elsevier, Amsterdam 1998, 295-312

Findlay JM, Gilchrist ID: Active vision: the psychology of looking and seeing. Oxford University Press, Oxford 2003

Folta K, Mähler C (2010): Schnelle Augenbewegungen und visuelle Fixation bei Kindern mit ADHS. Kindh Entwickl 20, 21-30

Folta-Schoofs K, Hilke C, Bethge H-J, Felbel D: Attention Deficit Hyperactivity Disorder: Precise Time Reproduction, but Accelerated Saccadic Peak Velocity of Voluntary Eye Movements after MPH-Medication. In: Heinen T (Hrsg.): Advances in Visual Perception Research. Nova Science Publishers Inc., New York 2015, 107-140

Fox PC (2004): Salivary enhancement therapies. Caries Res $\underline{38}, 241-246$

Franke M-K: Der Konsument: Homo Emoticus statt Homo Oeconomicus? Gabler Verlag, Wiesbaden 2014

Friedman A, Liebert LS: On the Time Course of Viewing Picture With a View Towards Remembering. In: Fisher DF, Monty RA, Senders JW (Hrsg.): Eye movements: cognition and visual perception. Lawrence Erlbaum Associates, Hillsdale 1981, 137-155 
Green RM, Hartles RL (1969): The effect of diets containing different monoand disaccharides on the incidence of dental caries in the albino rat. Arch Oral Biol 14, 235-241

Gustafsson BE, Quensel CE, Lanke LS, Lundqvist C, Grahnen H, Bonow BE, Krasse B (1954): The Vipeholm dental caries study; the effect of different levels of carbohydrate intake on caries activity in 436 individuals observed for five years. Acta Odontol Scand 11, 232-364

Hall JK, Hutton SB, Morgan MJ (2010): Sex differences in scanning faces: Does attention to the eyes explain female superiority in facial expression recognition? Cogn Emot 24, 629-637

Hallett KB, O'Rourke PK (2003): Social and behavioural determinants of early childhood caries. Aust Dent J $\underline{48}, 27-33$

Hassebrauck M (1983): Die Beurteilung der physischen Attraktivität: Konsens unter Urteilern? Z Sozialpsychol 14, 152-161

Haworth R, Sobey S, Chorney JM, Bezuhly M, Hong P (2015): Measuring attentional bias in children with prominent ears: A prospective eye-tracking study. J Plast Reconstr Aesthetic Surg $\underline{68}$, 1662-1666

He P, Kowler E (1992): The role of saccades in the perception of texture patterns. Vision Res $\underline{32}, 2151-2163$

Henderson JM, Pollatsek A, Rayner K (1989): Covert visual attention and extrafoveal information use during object identification. Percept Psychophys $\underline{45}, 196-208$

Henderson JM, McClure KK, Pierce S, Schrock G (1997): Object identification without foveal vision: Evidence from an artificial scotoma paradigm. Percept Psychophys $\underline{59}$, 323-346

Henderson JM, Williams CC, Falk RJ (2005): Eye movements are functional during face learning. Mem Cognit $\underline{33}$, 98-106

Hendriks AW (1996): Vergence eye movements during fixations in reading. Acta Psychol 92, 131-151

Hickman L, Firestone AR, Beck FM, Speer S (2010): Eye fixations when viewing faces. J Am Dent Assoc 141, 40-46

Hirsch C, John M, Waurick M (2000): Pilotstudie zur mundgesundheitsbezogenen Lebensqualität bei Kindern. Oralprophylaxe 22, $144-149$

Hirzel H-K: Zur Telemetrie des pH's im Interdentalraum. Juris Verlag, Zürich 1969

Hoffman JE: Visual attention and eye movements. In: Pashler H (Hrsg.): Attention. Psychology Press, London 1998, 119-154

Hojo K, Nagaoka S, Ohshima T, Maeda N (2009): Bacterial Interactions in Dental Biofilm Development. J Dent Res $\underline{88}$, 982-990

Holbrook WP, Árnadóttir IB, Takazoe I, Birkhed D, Frostell G (1995): Longitudinal study of caries, cariogenic bacteria and diet in children just before and after starting school. Eur J Oral Sci 103, 42-45 
Howells DJ, Shaw WC (1985): The validity and reliability of ratings of dental and facial attractiveness for epidemiologic use. Am J Orthod 88, 402-408

Hubel DH: Eye, brain, and vision. Scientific American Library, New York 1995

Huestegge L, Kunert H-J, Radach R (2010): Long-term effects of cannabis on eye movement control in reading. Psychopharmacology (Berl) 209, 77-84

Irtel H (2007): PXLab: The Psychological Experiments Laboratory. http://www.pxlab.de; Zugriff am 02.06.2016

Itti L, Koch C (2001): Computational modelling of visual attention. Nat Rev Neurosci 2, 194-203

Jacobi A: The dentition and its derangements. Course of lectures delivered in the New York medical college. Baillière Brothers, New York 1862

Jay P (1946): Fluorine and Dental Caries. J Am Dent Assoc 33, 489-495

Joos M, Rötting M, Velichkovsky BM: Spezielle Verfahren I: Bewegungen des menschlichen Auges: Fakten, Methoden und innovative Anwendungen. In: Rickheit G, Herrmann T, Deutsch W (Hrsg.): Psycholinguistik. Ein internationales Handbuch. Walter de Gruyter, Berlin 2003, 142-168

Just MA, Carpenter PA (1980): A theory of reading: from eye fixations to comprehension. Psychol Rev 87, 329-354

Karsh R, Breitenbach FW: Looking at Looking: The Amorphous Fixation Measure. In: Groner R (Hrsg.): Eye movements and psychological functions: international views. Lawrence Erlbaum Associates, Hillsdale 1983, 53-64

Karunakaran T, Gilbert D, Asimakopoulou K, Newton T (2011): The influence of visible dental caries on social judgements and overall facial attractiveness amongst undergraduates. J Dent 39, 212-217

Kaste LM (1995): Inappropriate Infant Bottle Feeding: Status of the Healthy People 2000 Objective. Arch Pediatr Adolesc Med 149, 786-791

Kershaw S, Newton JT, Williams DM (2008): The influence of tooth colour on the perceptions of personal characteristics among female dental patients: comparisons of unmodified, decayed and „whitened“ teeth. Br Dent J $\underline{204}$, 256-257

Kidd EAM, Thylstrup A, Fejerskov O, Bruun C (1980): Influence of Fluoride in Surface Enamel and Degree of Dental Fluorosis on Caries Development in vitro. Caries Res 14, 196-202

Kleiner M, Brainard D, Pelli D, Ingling A, Murray R, Broussard C (2007): What's new in Psychtoolbox-3? Perception 36 ECVP Supplement, 14

Knappwost A (1951): Zur Kinetik der Bildung von Hydroxylapatitdeckschichten auf Zahnschmelzoberflächen. Z Elektrochem Angew P $\underline{55}$, 586-590

Knappwost A (1956): Fluor-Hydroxyl-Substitution am Hydroxylapatit als lonenaustauschreaktion und ihre Anwendung für die Mikrofluoranalyse. Angew Chem 으, 371-373

Kokich VO, Kiyak HA, Shapiro PA (1999): Comparing the perception of dentists and lay people to altered dental esthetics. J Esthet Dent 11, 311-324 
Kokich VO, Kokich VG, Kiyak HA (2006): Perceptions of dental professionals and laypersons to altered dental esthetics: asymmetric and symmetric situations. Am J Orthod Dentofacial Orthop $\underline{130}$, 141-151

Krauskopf J, Cornsweet TN, Riggs LA (1960): Analysis of eye movements during monocular and binocular fixation. J Opt Soc Am 므, 572-578

Kroeber-Riel W: Bilder sind schnelle Schüsse ins Gehirn. Wirkungsgesetzte der Bildkommunikations. In: Randa-Campani S (Hrsg.): Wunderbare Werbe Welten: Marken, Macher, Mechanismen. Edition Braus, Heidelberg 2001, 112-117

Kroeber-Riel W, Diller H, Köhler R: Strategie und Technik der Werbung: Verhaltenswissenschaftliche und neurowissenschaftliche Erkenntnisse. Kohlhammer, Stuttgart 2015

Lamansky S (1869): Bestimmung der Winkelgeschwindigkeit der Blickbewegung, respective Augenbewegung. Pflugers Arch 2, 418-422

Lambrou D, Larsen MJ, Fejerskov O, Tachos B (1981): The Effect of Fluoride in Saliva on Remineralization of Dental Enamel in Humans. Caries Res $\underline{15}$, 341-345

Lang PJ: Behavioral treatment and bio-behavioral assessment: computer applications. In: Joseph B. Sidowski, Johnson JH, Williams TA (Hrsg.): Technology in mental health care delivery systems. Ablex Publishing Corporation, Norwood 1980, 119-137

Larsen MJ, Bruun C: Enamel/saliva - inorganic chemical reactions. In: Thylstrup A, Fejerskov $\mathrm{O}$ (Hrsg.): Textbook of cariology. Munksgaard, Kopenhagen 1986, 181-202

Laurentius MA: A discourse of the preseruation of the sight: of melancholike diseases; of rheumes, and of old age. Felix Kingston, London 1599

Lilienthal B, Goldsworthy NE, Sullivan HR, Cameron DA (1953): The biology of the children of Hopewood House, Bowral, New South Wales. I. Observations on dental caries extending over five years. Med J Aust 1 , 878-881

Lin J, Raghavan S, Fuerstenau DW (1981): The adsorption of fluoride ions by hydroxyapatite from aqueous solution. Colloids Surf $\underline{3}, 357-370$

Linz C, Gerdes ABM, Meyer-Marcotty P, Müller-Richter U, Böhm H, Ernestus RI, Kübler A, Alpers GW, Schweitzer T (2016): Perception of children's faces with unilateral coronal synostosis-an eye-tracking investigation. Childs Nerv Syst 32, 135-141

Litschel R, Majoor J, Tasman A (2015): Effect of protruding ears on visual fixation time and perception of personality. JAMA Facial Plast Surg $\underline{17}$, 183-189

Liversedge SP, White SJ, Findlay JM, Rayner K (2006): Binocular coordination of eye movements during reading. Vision Res $\underline{46}, 2363-2374$

Loftus GR (1972): Eye fixations and recognition memory for pictures. Cogn Psychol $\underline{3}, 525-551$

Loftus GR, Mackworth NH (1978): Cognitive determinants of fixation location during picture viewing. J Exp Psychol Hum Percept Perform 4, 565-572 
Marchant S, Brailsford SR, Twomey AC, Roberts GJ, Beighton D (2001): The predominant microflora of nursing caries lesions. Caries Res $\underline{35}, 397-406$

Marsh PD (1994): Microbial Ecology of Dental Plaque and its Significance in Health and Disease. Adv Dent Res $\underline{8}, 263-271$

McCann HG (1968): The solubility of fluorapatite and its relationship to that of calcium fluoride. Arch Oral Biol 13, 987-1001

Meissner CA, Brigham JC (2001): Thirty years of investigating the own-race bias in memory for faces: A meta-analytic review. Psychol Public Policy Law 7, 3-35

Menghini G, Steiner M, Imfeld T (2008): Kleinkinderkaries - Fakten und Vorbeugung. Ther Umsch $\underline{65}, 75-82$

Meyer-Marcotty P, Alpers GW, Gerdes ABM, Stellzig-Eisenhauer A (2010a): How others perceive orthognathic patients: an eye-tracking study. World $\mathrm{J}$ Orthod 11, 153-159

Meyer-Marcotty P, Gerdes ABM, Reuther T, Stellzig-Eisenhauer A, Alpers GW (2010b): Persons with Cleft Lip and Palate Are Looked at Differently. J Dent Res $\underline{89}, 400-404$

Meyer-Marcotty P, Gerdes ABM, Stellzig-Eisenhauer A, Alpers GW (2011): Visual Face Perception of Adults With Unilateral Cleft Lip and Palate in Comparison to Controls - An Eye-Tracking Study. Cleft Palate Craniofac J $\underline{48}, 210-216$

Mickasch HD, Haack J: Blickbewegungsforschung - Einführung in die physiologischen Grundlagen, Techniken und die Problem- und Anwendungsbereiche. In: Issing LJ, Mickasch HD, Haack J (Hrsg.): Blickbewegung und Bildverarbeitung: kognitionspsychologische Aspekte visueller Informationsverarbeitung. Peter Lang, Frankfurt a. M. 1986, 11-36

Moser A, Heide W, Kömpf D (1998): The effect of oral ethanol consumption on eye movements in healthy volunteers. J Neurol $245,542-550$

Mowrer OH, Ruch TC, Miller NE (1935): The corneo-retinal potential difference as the basic of teh galvonometric method of recording eye movements. Am J Physiol 114, 423-428

Naidu R, Nunn J, Donnelly-Swift E (2016): Oral health-related quality of life and early childhood caries among preschool children in Trinidad. BMC Oral Health $\underline{16}, 128$

Naujoks R, Schade H, Zelinka F (1967): Chemical Composition of Different Areas of the Enamel of Deciduous and Permanent Teeth (The content of $\mathrm{Ca}, \mathrm{P}, \mathrm{CO}_{2}, \mathrm{Na}$ and $\mathrm{N}_{2}$ ). Caries Res 1 , 137-143

Nicholls MER, Hobson A, Petty J, Churches O, Thomas NA (2017): The effect of cerebral asymmetries and eye scanning on pseudoneglect for a visual search task. Brain Cogn 111, 134-143

Northway WM (2000): The not-so-harmless maxillary primary first molar extraction. J Am Dent Assoc 131, 1711-1720

Nowak AJ, Casamassimo PS, Scott J, Moulton R (2014): Do early dental visits reduce treatment and treatment costs for children? Pediatr Dent $\underline{36}$, 489493 
Nummenmaa L, Hyönä J, Calvo MG (2006): Eye movement assessment of selective attentional capture by emotional pictures. Emotion $\underline{6}$, 257-268

Nyström M, Holmqvist K (2010): An adaptive algorithm for fixation, saccade, and glissade detection in eyetracking data. Behav Res Methods 42, 188204

Ogasawara T, Watanabe T, Kasahara H (1992): Readiness for toothbrushing of young children. ASDC J Dent Child 59, 353-359

Oldfield RC (1971): The assessment and analysis of handedness: the Edinburgh inventory. Neuropsychologia $\underline{9}$, 97-113

O'Neill C, Worthington HV, Donaldson M, Birch S, Noble S, Killough S, Murphy L, Greer M, Brodison J, Verghis R, Tickle M (2017): Cost-Effectiveness of Caries Prevention in Practice: A Randomized Controlled Trial. J Dent Res 96, 875-880

Oosterhaven SP, Westert GP, Schaub RM (1989): Perception and significance of dental appearance: the case of missing teeth. Community Dent Oral Epidemiol 17, 123-126

Oster PJ, Stern JA: Measurement of Eye Movement. In: Martin I, Venables PH (Hrsg.): Techniques in psychophysiology. John Wiley \& Sons, Chichester 1980, 275-327

Paris S, Meyer-Lückel H: Paradigmenwechsel. In: Meyer-Lückel H, Paris S, Ekstrand KR (Hrsg.): Karies: Wissenschaft und Klinische Praxis. Georg Thieme Verlag, Stuttgart 2012, 71-75

Pelli DG (1997): The VideoToolbox software for visual psychophysics: transforming numbers into movies. Spat Vis $\underline{10}$, 437-442

Pelphrey KA, Sasson NJ, Reznick JS, Paul G, Goldman BD, Piven J (2002): Visual scanning of faces in autism. J Autism Dev Disord 32, 249-261

Pieper K: Epidemiologische Begleituntersuchungen zur Gruppenprophylaxe 2000: Gutachten aus den Bundesländern bzw. Landesteilen BadenWürttemberg. Berlin. Brandenburg. Bremen. Hamburg. Hessen. Mecklenburg-Vorpommern. Niedersachsen. Nordrhein. Rheinland-Pfalz. Sachsen-Anhalt. Schleswig-Holstein. Thüringen. Westfalen. Deutsche Arbeitsgemeinschaft für Jugendzahnpflege, Bonn 2001

Pieper K: Epidemiologische Begleituntersuchungen zur Gruppenprophylaxe 2009: Gutachten aus den Bundesländern bzw. Landesteilen BadenWürttemberg. Bayern. Berlin. Brandenburg. Bremen. Hamburg. Hessen. Mecklenburg-Vorpommern. Niedersachsen. Nordrhein. Rheinland-Pfalz. Saarland. Sachsen. Sachsen-Anhalt. Schleswig-Holstein. Thüringen. Westfalen-Lippe. Deutsche Arbeitsgemeinschaft für Jugendzahnpflege, Bonn 2010

Pieper K, Dressler S, Heinzel-Gutenbrunner M, Neuhäuser A, Krecker M, Wunderlich K, Jablonski-Momeni A (2012): The influence of social status on pre-school children's eating habits, caries experience and caries prevention behavior. Int J Public Health $\underline{57}$, 207-215

Posner MI (1980): Orienting of attention. Q J Exp Psychol 32, 3-25 
Richards MR, Fields HW, Beck FM, Firestone AR, Walther DB, Rosenstiel S, Sacksteder JM (2015): Contribution of malocclusion and female facial attractiveness to smile esthetics evaluated by eye tracking. Am J Orthod Dentofacial Orthop 147, 472-482

Ritzmann-Fuchs C, Krämer N (2008): Behandlung unter Allgemeinanästhesie in der Kinderzahnheilkunde. Quintessenz 59, 1073-1083

Roberts G, Holzel H (2002): Intravenous antibiotic regimens and prophylaxis of odontogenic bacteraemia. Br Dent J $\underline{193}, 525-527$

Robinson J: Edinburgh Handedness Inventory. In: Volkmar FR (Hrsg.): Encyclopedia of Autism Spectrum Disorders. Springer, New York 2013, 1051-1054

Robke FJ (2008): Effects of Nursing Bottle Misuse on Oral Health: Prevalence of Caries, Tooth Malalignments and Malocclusions in North-German Preschool Children. J Orofac Orthop $\underline{69}, 5-19$

Rötting M: Typen und Parameter von Augenbewegungen. In: Rötting M, Seifert $\mathrm{K}$ (Hrsg.): Blickbewegungen in der Mensch-Maschine-Systemtechnik. Band 8. Pro Universitate Verlag, Sinzheim 1999a, 1-18

Rötting M: Methoden zur Registrierung von Augenbewegungen. In: Rötting M, Seifert $\mathrm{K}$ (Hrsg.): Blickbewegungen in der Mensch-MaschineSystemtechnik. Band 8. Pro Universitate Verlag, Sinzheim 1999b, 19-34

Russell JA (1980): A circumplex model of affect. J Pers Soc Psychol $\underline{39}, 1161-$ 1178

Scheinin A, Mäkinen KK (1976): Turku sugar studies. An overview. Acta Odontol Scand $\underline{34}, 405-408$

Schiffner U: Fünfte Deutsche Mundgesundheitsstudie (DMS V). Deutscher Zahnärzte Verlag, Köln 2016

Schmidt-Nielsen B (1946): The solubility of tooth substance in relation to the composition of saliva. Acta Odontol Scand Suppl 2, 1-88

Schmöger E: Klinische Elektroretinographie. In: Velhagen K (Hrsg.): Der Augenarzt. Band 2. 2. Auflage; Georg Thieme Verlag, Leipzig 1972, 571712

Schurgin MW, Nelson J, lida S, Ohira H, Chiao JY, Franconeri SL (2014): Eye movements during emotion recognition in faces. J Vis $\underline{14}, 14-14$

Schwendicke F, Dörfer CE, Schlattmann P, Page LF, Thomson WM, Paris S (2015): Socioeconomic Inequality and Caries: A Systematic Review and Meta-Analysis. J Dent Res $\underline{94}, 10-18$

SensoMotoric Instruments GmbH: iView X System Manual Version 2.8. o. Verl., o. Ort 2014

Shackel B: Eye movement recording by electro-oculography. In: Venables $\mathrm{PH}$, Martin I (Hrsg.): A manual of psychophysiological methods. North-Holland Publ. Co., Amsterdam 1967, 299-334

Shaw JH, Krumins I, Gibbons RJ (1967): Comparison of sucrose, lactose, maltose and glucose in the causation of experimental oral diseases. Arch Oral Biol 12, 755-768 
Shaw WC (1981): The influence of children's dentofacial appearance on their social attractiveness as judged by peers and lay adults. Am J Orthod $\underline{79}$, 399-415

Sheth BR, Pham T (2008): How emotional arousal and valence influence access to awareness. Vision Res $\underline{48}, 2415-2424$

Shwartz M, Gröndahl HG, Pliskin JS, Boffa J (1984): A longitudinal analysis from bite-wing radiographs of the rate of progression of approximal carious lesions through human dental enamel. Arch Oral Biol 29, 529-536

Sibert LE, Jacob RJK: Evaluation of eye gaze interaction. $\mathrm{CHI}$ '00 Proceedings of the SIGCHI conference on Human Factors in Computing Systems; ACM Press, Den Haag 2000, 281-288

Silva JBS, Cristino ED, Almeida NL de, Medeiros PCB de, Santos NA dos (2017): Effects of acute alcohol ingestion on eye movements and cognition: A double-blind, placebo-controlled study. PLOS ONE 12, e0186061

Smith FA, Ekstrand J: The occurrence and the chemistry of fluoride. In: Fejerskov O, Ekstrand J, Burt BA (Hrsg.): Fluoride in dentistry. 2. Auflage; Munksgaard, Kopenhagen 1996, 17-26

Somani A, Newton JT, Dunne S, Gilbert DB (2010): The impact of visible dental decay on social judgements: comparison of the effects of location and extent of lesion. Int Dent J $\underline{60}, 169-174$

Sønju Clasen AB, Øgaard B, Duschner H, Ruben J, Arends J, Sönju T (1997): Caries Development in Fluoridated and Non-Fluoridated Deciduous and Permanent Enamel in Situ Examined by Microradiography and Confocal Laser Scanning Microscopy. Adv Dent Res 11, 442-447

Staehle HJ, Schiffner U, Dörfer CE (2007): Häusliche mechanische Zahn- und Mundpflege - Stellungnahmen der Deutschen Gesellschaft für Zahn-, Mundund Kieferheilkunde (DGZMK). Dtsch Zahnärztl Z $\underline{62}$, 616-621

Stambaugh RV, Wittrock JW (1977): The relationship of the pulp chamber to the external surface of the tooth. J Prosthet Dent $\underline{37}, 537-546$

Steinfurth E, Wendt J, Hamm A (2013): Neurobiologische Grundlagen der Emotionsregulation. Psychol Rundsch 누, 208-216

Sumikawa DA, Marshall GW, Gee L, Marshall SJ (1999): Microstructure of primary tooth dentin. Pediatr Dent 21, 439-444

Takahashi N, Nyvad B (2008): Caries Ecology Revisited: Microbial Dynamics and the Caries Process. Caries Res $\underline{42}, 409-418$

Takahashi N, Nyvad B (2016): Ecological Hypothesis of Dentin and Root Caries. Caries Res $\underline{50}, 422-431$

Tan KW, Stephen ID (2013): Colour detection thresholds in faces and colour patches. Perception 42, 733-741

ten Cate JM, Featherstone JDB (1991): Mechanistic Aspects of the Interactions Between Fluoride and Dental Enamel. Crit Rev Oral Biol Med 2, 283-296

Thylstrup A (1990): Clinical Evidence of the Role of Pre-eruptive Fluoride in Caries Prevention. J Dent Res $\underline{69}$, 742-750 
Tschammler C, Zimmermann D, Batschkus S, Wiegand A, Folta-Schoofs K (2018): Perception of children with visible untreated and treated caries. J Dent 74, 37-42

Tschoppe J, Kielbassa AM, Tschoppe P (2012): Kinderprophylaxe von Anfang an. ZWP Spezial $\underline{12}, 4-10$

Tversky B (1974): Eye fixations in prediction of recognition and recall. Mem Cognit 2, 275-278

Umweltbundesamt (2017): Bekanntmachung der Liste der Aufbereitungsstoffe und Desinfektionsverfahren gemäß $\S 11$ der Trinkwasserverordnung - 19 . Änderung - $\quad$ (Stand: Dezember 2017). https://www.umweltbundesamt.de/sites/default/files/medien/421/dokumente/ 19._bekanntmachung_der_liste_der_aufbereitungsstoffe_und_desinfektions verfahren_gemaess_ss_11_trinkwv_2001.pdf; Zugriff am 19.02.2018

van Schijndel O, Litschel R, Maal TJJ, Bergé SJ, Tasman A-J (2015): Eye tracker based study: Perception of faces with a cleft lip and nose deformity. J Craniomaxillofac Surg $\underline{43}$, 1620-1625

Vassallo S, Cooper SL, Douglas JM (2009): Visual scanning in the recognition of facial affect: Is there an observer sex difference? J Vis $\underline{9}, 11-11$

Velichkovsky BM, Sprenger A, Pomplun M: Auf dem Weg zur Blickmaus: Die Beeinflussung der Fixationsdauer durch kognitive und kommunikative Aufgaben. In: Liskowsky R, Velichkovsky BM, Wünschmann W (Hrsg.): Software-Ergonomie '97: Usability Engineering: Integration von MenschComputer-Interaktion und Software-Entwicklung; Vieweg+Teubner Verlag, Wiesbaden 1997, 317-327

Verma D, Garg PK, Dubey AK (2018): Insights into the human oral microbiome. Arch Microbiol 200, 525-540

Verordnung über die Qualität von Wasser für den menschlichen Gebrauch (Trinkwasserverordnung - TrinkwV): 2018

Volkmann FC (1962): Vision during Voluntary Saccadic Eye Movements. J Opt Soc Am $\underline{52}, 571$

von Helmholtz H: Handbuch der Physiologischen Optik. Band 2. 3. Auflage; Voss, Hamburg 1910a

von Helmholtz $\mathrm{H}$ : Handbuch der Physiologischen Optik. Band 3. 3. Auflage; Voss, Hamburg 1910b

Wagner Y, Heinrich-Weltzien R (2016): Evaluation of a regional German interdisciplinary oral health programme for children from birth to 5 years of age. Clin Oral Investig 21, 225-235

Walker-Smith GJ, Gale AG, Findlay JM (1977): Eye Movement Strategies Involved in Face Perception. Perception $\underline{6}$, 313-326

Warren JJ, Weber-Gasparoni K, Marshall TA, Drake DR, Dehkordi-Vakil F, Kolker JL, Dawson DV (2008): Factors Associated with Dental Caries Experience in 1-Year-Old Children. J Public Health Dent $\underline{68}$, 70-75

Wetzel W-E (1982): „Zuckertee-Karies“ als Folge exzessiven Genusses von Fertigtees aus Saugerfläschchen. Monatsschr Kinderheilkd 130, 726-730 
Wetzel WE (1986): Folgen apikaler Milchzahnerkrankungen auf Mineralisation und Durchbruch bleibender Zähne. Dtsch Zahnarztl Z 41, 179-181

Wetzel W-E (1988): Nursing-Bottle-Syndrom (NBS) bei Kleinkindern: Gebißbefunde, Häufigkeit und familiäre Bedingungen. Monatsschr Kinderheilkd 136, 673-679

Willerhausen B, Blettner M, Kasaj A, Hohenfellner K (2007): Association between body mass index and dental health in 1,290 children of elementary schools in a German city. Clin Oral Investig 11, 195-200

Wilson PR, Beynon AD (1989): Mineralization differences between human deciduous and permanent enamel measured by quantitative microradiography. Arch Oral Biol $\underline{34}, 85-88$

Wolf R, Wolf D: Vom Sehen zum Wahrnehmen: Aus Illusionen entsteht ein Bild der Wirklichkeit. In: Maelicke A (Hrsg.): Vom Reiz der Sinne. VCH Verlag, Weinheim 1990, 47-73

Wong L, Cutress TW, Duncan JF (1987): The Influence of Incorporated and Adsorbed Fluoride on the Dissolution of Powdered and Pelletized Hydroxyapatite in Fluoridated and Non-fluoridated Acid Buffers. J Dent Res 66, 1735-1741

Wyne AH (1999): Early childhood caries: nomenclature and case definition. Community Dent Oral Epidmiol 27, 313-315

Yarbus AL: Eye Movements and Vision. Plenum Press, New York 1967

Young LR, Sheena D (1975): Survey of eye movement recording methods. Behav Res Methods 프, 397-429

Zero DT (2004): Sugars - The Arch Criminal? Caries Res $\underline{38}, 277-285$

Zwiauer K, Österreichische Gesellschaft für Kinder- und Jugendheilkunde (ÖGKJ), Österreichische Gesellschaft für Kinderzahnheilkunde (ÖGK) (2011): Karies- und Fluoridprophylaxe bei Kindern und Jugendlichen in Österreich: Stellungnahme der Ernährungskommission der Österreichischen Gesellschaft für Kinder- und Jugendheilkunde (ÖGKJ) und der Österreichischen Gesellschaft für Kinderzahnheilkunde (ÖGK). Monatsschrift Kinderheilkunde 159, 1128-1132 


\section{Genehmigungsnachweise}

Irtel H (2007): PXLab: The Psychological Experiments Laboratory (Version 2.1.11). Stand 19.06.2007. http://www.pxlab.de; Zugriff am 02.06.2016. Die Verwendung erfolgt mit freundlicher Genehmigung des Autors.

Tschammler C, Zimmermann D, Batschkus S, Wiegand A, Folta-Schoofs K (2018): Perception of children with visible untreated and treated caries. Journal of Dentistry 74, 37-42. doi.org/10.1016/j.jdent.2018.05.006. Die Verwendung erfolgt mit freundlicher Genehmigung des Elsevier Verlags: Elsevier Limited (Branch Office), The Officers' Mess Business Centre, Royston Road, Duxford, Cambridge CB22 4QH, United Kingdom. 


\section{Anhang}

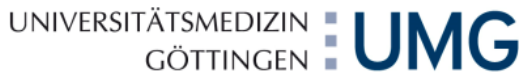

Univ.-Prof. Dr.. A. Wiegand

37099 Göttingen-Briefpos

Robert-Koch-Straße 40, 37075 Göttingen-Adresse
0551 / 39-22884 Telefon
0551 / 39-12616 Fax

annette.wiegand@med.uni-goettingen.de E-Mail

Leiterin der Studie: Prof. Dr. A. Wiegand

Studienkoordination (Göttingen): Dr. Claudia Tschammler

Studienkoordination (Hildesheim), Laborleitung: Prof. Dr. K. Folta-Schoofs

\section{Eye-Tracking-Studie}

Fragebogen (Version 1.0)

Studienteilnehmer-Nr.

Heutiges Datum:

Bitte füllen Sie diesen Fragebogen vollständig aus. Bei Fragen können Sie sich jederzeit an unsere(n) Mitarbeiter/in wenden.

Geburtsdatum: Alter:

Geschlecht:

$\square$ männlich $\square$ weiblich

Studienfach: Universität:

Fachsemester:

\section{Exklusionskriterien}

1. Alter $<18$ Jahre

2. Linkshänder

3. Kurz-oder Weitsichtig (Nichtzutreffendes bitte streichen)

4. Allgemeinerkrankungen (Welche?

5. neurologische oder psychische (Vor)Erkankung/en? (Welche?

6. aktuell in (zahn)ärztlicher Behandlung? (Wegen? ...

7. regelmäßige Medikamenteneinnahme? (Welche? ..

8. Medikamenten- oder Drogeneinnahme in den letzten 7 Tagen?

(Welche? Wann? 


\section{Danksagung}

Mein besonderer Dank gilt Frau Prof. Dr. Annette Wiegand, der Direktorin der Poliklinik für Präventive Zahnmedizin, Parodontologie und Kariologie der Universitätsmedizin Göttingen, für die Überlassung des Themas sowie die überaus engagierte Betreuung.

Frau Dr. Claudia Tschammler, Mitarbeiterin der Poliklinik für Präventive Zahnmedizin, Parodontologie und Kariologie der Universitätsmedizin Göttingen, danke ich für die enge und herausragende unterstützende Hilfe.

Des Weiteren danke ich Herrn Prof. Dr. Kristian Folta-Schoofs, dem Leiter der Arbeitsgruppe Neurodidaktik am Institut für Psychologie der Stiftung Universität Hildesheim, für die gute Zusammenarbeit und die Nutzung seiner Forschungseinrichtungen.

Den Mitarbeiterinnen und Mitarbeitern der Zentralen Serviceeinheit für Medizinische Biometrie und Statistische Bioinformatik am Institut für Medizinische Statistik der Universitätsmedizin Göttingen danke ich für die intensive Beratung.

Allen teilnehmenden Zahnärztinnen und Zahnärzten der Poliklinik für Präventive Zahnmedizin, Parodontologie und Kariologie der Universitätsmedizin Göttingen danke ich recht herzlich für die Unterstützung der Studie.

Ein weiterer Dank gilt allen Probandinnen für ihre Bereitschaft und die Unterstützung dieses Forschungsvorhabens.

Abschließend möchte ich mich sehr herzlich bei allen teilnehmenden Patientinnen und Patienten für die Teilnahme an der Studie bedanken. 